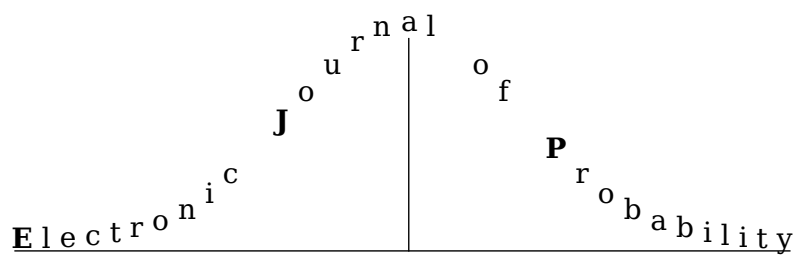

Electron. J. Probab. 27 (2022), article no. 16, 1-54.

ISSN: 1083-6489 https://doi.org/10.1214/22-EJP741

\title{
Scaling limits of tree-valued branching random walks*
}

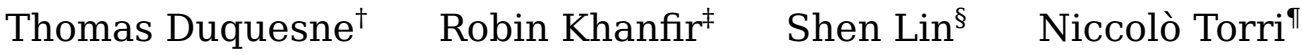

\begin{abstract}
We consider a branching random walk (BRW) taking its values in the b-ary rooted tree $W_{b}$ (i.e. the set of finite words written in the alphabet $\{1, \ldots, b\}$, with $b \geq 2$ ). The BRW is indexed by a critical Galton-Watson tree conditioned to have $n$ vertices; its offspring distribution is aperiodic and is in the domain of attraction of a $\gamma$-stable law, $\gamma \in(1,2]$. The jumps of the BRW are those of a nearest-neighbour null-recurrent random walk on $W_{b}$ (reflection at the root of $W_{b}$ and otherwise: probability $1 / 2$ to move closer to the root of $\mathbb{W}_{\mathrm{b}}$ and probability $1 /(2 \mathrm{~b})$ to move away from it to one of the $\mathrm{b}$ sites above). We denote by $\mathcal{R}_{\mathrm{b}}(n)$ the range of the BRW in $\mathbb{W}_{\mathrm{b}}$ which is the set of all sites in $\mathbb{W}_{\mathrm{b}}$ visited by the BRW. We first prove a law of large numbers for $\# \mathcal{R}_{\mathrm{b}}(n)$ and we also prove that if we equip $\mathcal{R}_{\mathrm{b}}(n)$ (which is a random subtree of $W_{\mathrm{b}}$ ) with its graph-distance $d_{\mathrm{gr}}$, then there exists a scaling sequence $\left(a_{n}\right)_{n \in \mathbb{N}}$ satisfying $a_{n} \rightarrow \infty$ such that the metric space $\left(\mathcal{R}_{\mathrm{b}}(n), a_{n}^{-1} d_{\mathrm{gr}}\right)$, equipped with its normalised empirical measure, converges to the reflected Brownian cactus with $\gamma$-stable branching mechanism: namely, a random compact real tree that is a variant of the Brownian cactus introduced by N. Curien, J-F. Le Gall and G. Miermont in [7].
\end{abstract}

Keywords: branching random walks; Galton-Watson tree; scaling limit; superprocess; Brownian snake; Brownian cactus; real tree.

MSC2020 subject classifications: 60J80; 60G50; 60G52; 60F17.

Submitted to EJP on April 15, 2021, final version accepted on January 8, 2022.

\section{Introduction}

Since the seventies, branching random walk (BRW) is an area of research that is intensively studied and is linked to travelling wave solutions of semi-linear partial differential

\footnotetext{
${ }^{*} \mathrm{~N}$. Torri was supported by the project Labex MME-DII (ANR11-LBX-0023-01).

${ }^{\dagger}$ Sorbonne Université and Université de Paris, CNRS, Laboratoire de Probabilités, Statistique et Modélisation, F-75005 Paris, France. E-mail: thomas.duquesne@sorbonne-universite. $\mathrm{fr}$

‡Sorbonne Université and Université de Paris, CNRS, Laboratoire de Probabilités, Statistique et Modélisation, F-75005 Paris, France. E-mail: robin.khanfir@sorbonne-universite.fr

§Sorbonne Université and Université de Paris, CNRS, Laboratoire de Probabilités, Statistique et Modélisation, F-75005 Paris, France. E-mail: shen.lin@sorbonne- universite. $\mathrm{fr}$

"Université Paris-Nanterre, Laboratoire MODAL'X, UMR CNRS 9023 and FP2M, CNRS FR 2036, France. E-mail: ntorri@parisnanterre.fr
} 
equations (FKPP) or various models of statistical mechanics (Generalized random energy model, Mandelbrot's cascades, Gaussian free field): we refer to the book of Z. Shi [31] for an overview of this topic; we also refer to the works of S. Gouëzel, I. Huerter, S. Lalley and T. Sellke $[20,17,19,16]$ for the study of BRW in hyperbolic spaces and to T. Liggett [27] and to I. Benjamini and S. Müller [4] for branching random walks on trees. In most of the previous works, BRWs are indexed by an infinite supercritical Galton-Watson tree (GW-trees) and questions focus on various survival events or extremal behaviours of BRWs.

In this paper, we consider instead a BRW that takes its values in the b-ary tree $\mathbb{W}_{\mathrm{b}}$ and that is indexed by a critical Galton-Watson tree conditioned to have $n$ vertices. The jumps of the BRW are those of a nearest-neighbour null-recurrent RW on $\mathbb{W}_{\mathrm{b}}$. Namely, at the root of $\mathbb{W}_{\mathrm{b}}$ (that is denoted by $\varnothing$ ), it is reflected and elsewhere, with probability $1 / 2$, it jumps to the neighbour closer to the root of $\mathbb{W}_{b}$ and with probability $1 /(2 \mathrm{~b})$, it moves further from the root of $\mathbb{W}_{\mathrm{b}}$ and it jumps to one of the $\mathrm{b}$ sites above. We study the range $\mathcal{R}_{\mathrm{b}}(n)$ of this BRW when $n \rightarrow \infty$. More precisely, we first show that $\frac{1}{n} \# \mathcal{R}_{\mathrm{b}}(n)$ converges in probability to a constant; this law of large numbers is the analogue of the results due to J-F. Le Gall and L. $[23,24]$ who treat the cases of $\mathbb{Z}^{d}$-valued BRWs that are indexed by critical GW-trees conditioned to have $n$ vertices. We then prove that $\mathcal{R}_{\mathrm{b}}(n)$, seen as a subtree of the b-ary tree, converges, when it is suitably rescaled, to a continuum random tree called the reflected Brownian cactus with $\gamma$-stable branching mechanism: namely, a random compact real tree that is a variant of the Brownian cactus introduced by N. Curien, J-F. Le Gall and G. Miermont in [7].

This limit theorem for $\mathcal{R}_{\mathrm{b}}(n)$ is related to earlier works on scaling limits of the range of tree-valued critical or near-critical biased random walks (RWs): in particular we refer to D. [12] who deals with near-critical biased RWs on b-ary trees, to Y. Peres and O. Zeitouni [30] who show that the distance to the root of a critical biased RW in a Galton-Watson environment is diffusive, to A. Dembo and N. Sun [8] who study the cases of critical biased RWs on $N$-type GW-trees, to E. Aïdékon and L. de Raphélis [2] who improve Y. Peres and O. Zeitouni's result and who show that the range of the same RW converges when suitably rescaled, to a variant of the Brownian CRT, and to X. Chen and G. Miermont [6] who show that rescaled Brownian bridges and loops in hyperbolic spaces converge to the Brownian CRT. Their work is based on a previous results due to P. Bougerol and T. Jeulin [5]. Independently, A. Stewart shows in his PhD Thesis [32] that the rescaled simple RW bridges on a $d$-regular tree $(d \geq 3)$ converge to the Brownian CRT.

Let us describe more precisely the results that we obtain. We consider a (rooted and ordered) Galton-Watson tree $\tau$ with offspring distribution $\mu$ that satisfies the following: we fix $\gamma \in(1,2]$ and we assume

$$
\left\{\begin{aligned}
&\left(\mathrm{H}_{1}\right): \sum_{k \in \mathbb{N}} k \mu(k)=1, \\
&\left.\left(\mathrm{H}_{2}\right): \mu \text { is aperiodic (namely, } \mu \text { is not supported by a proper subgroup of } \mathbb{Z}\right), \\
&\left(\mathrm{H}_{3}\right): \text { Either } \gamma=2 \text { and } \sum_{k \in \mathbb{N}} k^{2} \mu(k)<\infty, \text { or } \gamma \in(1,2) \text { and } \mu \text { is in } \\
& \text { the domain of attraction of a } \gamma \text {-stable law. }
\end{aligned}\right.
$$

Note that $\left(\mathrm{H}_{1}\right)$ implies that a.s. the total number of vertices $\# \tau$ is finite; $\left(\mathrm{H}_{2}\right)$ implies that for all large enough integers $n, \mathbf{P}(\# \tau=n)>0$. We translate $\left(\mathrm{H}_{3}\right)$ into the following assertion: let $\left(L_{n}\right)_{n \in \mathbb{N}}$ be an i.i.d. sequence of $(\{-1\} \cup \mathbb{N})$-valued random variables such that $\mathbf{P}\left(L_{n}=k\right)=\mu(k+1), k \geq-1$ and let $X$ be a real random variable whose law is spectrally positive $\gamma$-stable; it is characterised by its Laplace exponent: $\log \mathbf{E}[\exp (-\lambda X)]=\lambda^{\gamma}$, $\lambda \in[0, \infty)$. Then under $\left(\mathrm{H}_{3}\right)$, there exists a nondecreasing $\frac{\gamma-1}{\gamma}$-regularly varying sequence 
$\left(a_{n}\right)_{n \in \mathbb{N}}$ such that

$$
\frac{a_{n}}{n}\left(L_{1}+\ldots+L_{n}-n\right) \underset{n \rightarrow \infty}{\stackrel{\text { (law) }}{\longrightarrow}} X .
$$

As we see below, under $(\mathbf{H}), \tau$ behaves regularly when it is conditioned to be large: namely, we see that suitably rescaled versions of $\tau$ under $\mathbf{P}(\cdot \mid \# \tau=n)$ converge in distribution when $n \rightarrow \infty$.

Conveniently, we view $\tau$ as a family tree whose ancestor is the root and where siblings are ordered by birth-rank. The depth-first exploration of $\tau$ is the sequence of vertices $\left(u_{k}\right)_{0 \leq k<\# \tau}$ that is defined recursively as follows: $u_{0}$ is the root and for all $k \in\{0, \ldots, \# \tau-2\}$, let $v$ be the most recent ancestor of $u_{k}$ having at least one unexplored child (note that possibly $v=u_{k}$ ); then $u_{k+1}$ is the unexplored child of $v$ with least birthrank. Our first result is the following law of large numbers for the size of the range of the $\mathbb{W}_{\mathrm{b}}$-valued $\tau$-indexed critical branching random walk (see Sections 2 and 3 for more precise definitions).

Theorem 1.1. Let $\tau$ be a Galton-Watson tree with offspring distribution $\mu$ that satisfies $(\mathbf{H})$. Recall that $\left(u_{k}\right)_{0 \leq k<\# \tau}$ stands for the depth-first exploration of $\tau$. Conditionally given $\tau$, let $\left(Y_{v}\right)_{v \in \tau}$ be a $\mathbb{W}_{\mathrm{b}}$-valued $\tau$-indexed critical branching random walk starting at $Y_{\text {root }}=\varnothing$. Then, there exists a constant $c_{\mu, \mathrm{b}} \in(0, \infty)$ that only depends on $\mu$ and $\mathrm{b}$ such that

$$
\forall \varepsilon \in(0, \infty), \quad \lim _{n \rightarrow \infty} \mathbf{P}\left(\frac{1}{n} \max _{1 \leq k \leq n}\left|\#\left\{Y_{u_{l}} ; 0 \leq l<k\right\}-c_{\mu, \mathrm{b}} k\right|>\varepsilon \mid \# \tau=n\right)=0,
$$

In particular, for all $\varepsilon \in(0, \infty)$, we get $\lim _{n \rightarrow \infty} \mathbf{P}\left(\left|\frac{1}{n} \# \mathcal{R}_{\mathrm{b}}-c_{\mu, \mathrm{b}}\right|>\varepsilon \mid \# \tau=n\right)=0$, where $\mathcal{R}_{\mathrm{b}}$ stands for the range $\left\{Y_{v} ; v \in \tau\right\}$ of the branching random walk.

Let $\tau,\left(Y_{v}\right)_{v \in \tau}$ and $\mathcal{R}_{\mathrm{b}}=\left\{Y_{v} ; v \in \tau\right\}$ be as in Theorem 1.1. Observe that $\mathcal{R}_{\mathrm{b}}$ is a subtree of $\mathbb{W}_{\mathrm{b}}$. Our second main result is a limit theorem for rescaled versions of the metric spaces $\left(\mathcal{R}_{\mathrm{b}}, d_{\mathrm{gr}}\right)$ where $d_{\mathrm{gr}}$ stands for the graph-distance. To state it, let us first recall a limit theorem for $\left(\tau, d_{\mathrm{gr}}\right)$. Set $H_{k}(\tau)=d_{\mathrm{gr}}\left(\right.$ root,$\left.u_{k}\right)$ for all $k \in\{0, \ldots, \# \tau-1\}$, that is the height process of $\tau$. Note that $H(\tau)=\left(H_{k}(\tau)\right)_{k \in\{0, \ldots, \# \tau-1\}}$ entirely codes $\tau$. Then, Theorem 3.1 in D. [11] asserts the following: assume $(\mathbf{H})$ as in (1.1) and let $\left(a_{n}\right)$ be as in (1.2); then there is a nonnegative continuous process $H=\left(H_{s}\right)_{s \in[0,1]}$ such that

$$
\left(\frac{1}{a_{n}} H_{\lfloor n s\rfloor}(\tau)\right)_{s \in[0,1]} \text { under } \mathbf{P}(\cdot \mid \# \tau=n) \underset{n \rightarrow \infty}{\longrightarrow} H,
$$

weakly on $\mathbf{C}([0,1], \mathbb{R})$. When $\gamma=2, H$ is the normalised Brownian excursion and this result is due to Aldous (see Theorem 23 in Aldous [3]). When $\gamma \in(1,2), H$ is the normalised excursion of the $\gamma$-stable height process that is a local-time function of a $\gamma$-stable spectrally positive Lévy process.

The metric space that is the limit of $\left(\tau, \frac{1}{a_{n}} d_{\mathrm{gr}}\right)$ as $n \rightarrow \infty$ is derived from the normalised excursion of the $\gamma$-stable height process $H$ as follows: for all $s_{1}, s_{2} \in[0,1]$, we set

$$
d_{H}\left(s_{1}, s_{2}\right)=H_{s_{1}}+H_{s_{2}}-2 \min _{s_{1} \wedge s_{2} \leq s \leq s_{1} \vee s_{2}} H_{s} .
$$

We easily check that a.s. $d_{H}$ is a pseudo-metric on $[0,1]$. We introduce the relation $\sim_{H}$ on $[0,1]$ by setting $s_{1} \sim_{H} s_{2}$ if and only if $d_{H}\left(s_{1}, s_{2}\right)=0$; clearly, $\sim_{H}$ is an equivalence relation and the normalised $\gamma$-stable Lévy tree is taken as the quotient space $T_{H}=[0,1] / \sim_{H}$, equipped with the distance induced by $d_{H}$ that we keep denoting $d_{H}$. We denote by $p_{H}:[0,1] \rightarrow T_{H}$ the canonical projection. Note that $p_{H}$ is continuous; therefore $T_{H}$ is compact and connected. Moreover, $T_{H}$ is a real tree, namely, a metric space such that all pairs of points are joined by a unique simple arc that turns out to be a geodesic (see Definition 4.6 for more details). We set $r_{H}:=p_{H}(0)$ that is viewed as the root of $T_{H}$ and 
we equip $T_{H}$ with the measure $\mu_{H}$ that is the image of the Lebesgue measure on $[0,1]$ via $p_{H}$, namely, $\int_{T_{H}} f d \mu_{H}=\int_{0}^{1} f\left(p_{H}(s)\right) d s$, for all continuous $f: T_{H} \rightarrow \mathbb{R}$. The convergence (1.4) then implies the following one.

$$
\left(\tau, \frac{1}{a_{n}} d_{\mathrm{gr}}, \text { root }, \frac{1}{n} \mathrm{~m}\right) \quad \text { under } \mathbf{P}(\cdot \mid \# \tau=n) \underset{n \rightarrow \infty}{\longrightarrow}\left(T_{H}, d_{H}, r_{H}, \mu_{H}\right)
$$

where $\mathrm{m}=\sum_{v \in \tau} \delta_{v}$ stands for the counting measure on $\tau$. Here the convergence holds weakly on the space $\mathrm{IM}$ of isometry classes of pointed measured compact metric spaces equipped with the Gromov-Hausdorff-Prokhorov distance $\boldsymbol{\delta}_{\mathrm{GHP}}$ that makes it a Polish space, as proved in Theorem 2.5 of R. Abraham, J-F. Delmas and P. Hoscheit [1]. (See (4.10) for a precise definition of $\boldsymbol{\delta}_{\mathrm{GHP}}$ and see (4.11) for more details.) For more details on Lévy trees see J-F. Le Gall and Y. Le Jan [25] and D. and J-F. Le Gall [14, 13] (see also Section 4.4).

The limit of rescaled versions of the metric spaces $\left(\mathcal{R}_{\mathrm{b}}, d_{\mathrm{gr}}\right)$ is constructed as follows: as proved in D. and J-F. Le Gall [13] (Lemma 6.4 p. 600, that is recalled in Lemma 4.32), conditionally given $H$, there exists a Hölder-continuous centered Gaussian process $\sigma \in T_{H} \longmapsto W_{\sigma} \in \mathbb{R}$ whose covariance is characterised by $\mathbf{E}\left[\left|W_{\sigma_{1}}-W_{\sigma_{2}}\right|^{2} \mid H\right]=d_{H}\left(\sigma_{1}, \sigma_{2}\right)$, for all $\sigma_{1}, \sigma_{2} \in T_{H}$. Then, we set

$$
\forall \sigma_{1}, \sigma_{2} \in T_{H}, \quad d_{H, W}\left(\sigma_{1}, \sigma_{2}\right)=\left|W_{\sigma_{1}}\right|+\left|W_{\sigma_{2}}\right|-2 \min _{\sigma \in \llbracket \sigma_{1}, \sigma_{2} \rrbracket}\left|W_{\sigma}\right|,
$$

where $\llbracket \sigma_{1}, \sigma_{2} \rrbracket$ is the unique geodesic that joins $\sigma_{1}$ to $\sigma_{2}$ in $T_{H}$. In Lemma 4.22 , we prove that $d_{H, W}$ is a pseudo-metric on $T_{H}$; we then define the equivalence relation $\sim_{H, W}$ on $T_{H}$ by setting $\sigma_{1} \sim_{H, W} \sigma_{2}$ if and only if $d_{H, W}\left(\sigma_{1}, \sigma_{2}\right)=0$ and we denote by $T_{H, W}=T_{H} / \sim_{H, W}$ the quotient metric space and we keep denoting by $d_{H, W}$ the resulting metric; we denote by $\pi_{H, W}: T_{H} \rightarrow T_{H, W}$ the canonical projection that is continuous. Thus $T_{H, W}$ is compact and connected, and $\left(T_{H, W}, d_{H, W}\right)$ is a real tree (see Section 4.6, especially Proposition 4.34, for more properties of $T_{H, W}$ ). It turns out that this kind of spaces has been introduced in N. Curien, J.-F. Le Gall and G. Miermont [7] (see also J.-F. Le Gall [22] for a different purpose); they coined the name Brownian cactus, so we call $\left(T_{H, W}, d_{H, W}\right)$ the normalised reflected Brownian cactus with $\gamma$-stable branching mechanism. We next set $r_{H, W}=\pi_{H, W}\left(r_{H}\right)$ that is viewed as the root of $T_{H, W}$ and we equip $T_{H, W}$ with the measure $\mu_{H, W}$ that is the image of $\mu_{H}$ via $\pi_{H, W}$ : namely, $\int_{T_{H, W}} f d \mu_{H, W}=\int_{T_{H}} f\left(\pi_{H, W}(\sigma)\right) \mu_{H}(d \sigma)$, for all continuous $f: T_{H, W} \rightarrow \mathbb{R}$. Our second result is the following limit theorem.

Theorem 1.2. Let $\tau$ be a Galton-Watson tree with offspring distribution $\mu$ that satisfies $(\mathbf{H})$ as in (1.1). We denote by $\mathrm{m}=\sum_{v \in \tau} \delta_{v}$ the counting measure on $\tau$ and we denote by $d_{\text {gr }}$ the graph distance on $\tau$. Conditionally given $\tau$, let $\left(Y_{v}\right)_{v \in \tau}$ be a $\mathbb{W}_{\mathrm{b}}$-valued $\tau$-indexed critical branching random walk starting at $Y_{\text {root }}=\varnothing$. We denote by $\mathcal{R}_{\mathrm{b}}=\left\{Y_{v} ; v \in \tau\right\}$ the range of $Y$, by $\mathbf{m}_{\mathrm{occ}}^{\mathrm{b}}=\sum_{v \in \tau} \delta_{Y_{v}}$ the occupation measure of $Y$ and by $d_{\mathrm{gr}}$ the graph distance on $\mathcal{R}_{\mathrm{b}}$. We denote by $\left(T_{H}, d_{H}, r_{h}, \mu_{H}\right)$ the normalised $\gamma$-stable Lévy tree and by $\left(T_{H, W}, d_{H, W}, r_{H, W}, \mu_{H, W}\right)$ the normalised reflected Brownian cactus with $\gamma$-stable branching mechanism as defined above. Let $\left(a_{n}\right)_{n \in \mathbb{N}}$ be as in (1.2). Then, the following limit holds weakly on $\left(\mathrm{M}, \delta_{\mathrm{GHP}}\right)^{2}$

$$
\begin{aligned}
\left(\left(\tau, \frac{1}{a_{n}} d_{\mathrm{gr}}, \text { root }, \frac{1}{n} \mathrm{~m}\right),\left(\mathcal{R}_{\mathrm{b}}, \frac{1}{\sqrt{a_{n}}} d_{\mathrm{gr}}, \varnothing, \frac{1}{n} \mathbf{m}_{\mathrm{occ}}^{\mathrm{b}}\right)\right) \text { under } \mathbf{P}(\cdot \mid \# \tau=n) \\
\stackrel{\text { (law) }}{\longrightarrow}\left(\left(T_{H}, d_{H}, r_{H}, \mu_{H}\right),\left(T_{H, W}, d_{H, W}, r_{H, W}, \mu_{H, W}\right)\right) .
\end{aligned}
$$

Moreover, denote by $\mathbf{m}_{\text {count }}^{\mathrm{b}}=\sum_{x \in \mathcal{R}_{\mathrm{b}}} \delta_{x}$ the counting measure on $\mathcal{R}_{\mathrm{b}}$; then, for all $\varepsilon \in(0, \infty)$,

$$
\lim _{n \rightarrow \infty} \mathbf{P}\left(d_{\text {Prok }}^{(n)}\left(\frac{c_{\mu, \mathrm{b}}}{n} \mathbf{m}_{\mathrm{occ}}^{\mathrm{b}}, \frac{1}{n} \mathbf{m}_{\text {count }}^{\mathrm{b}}\right)>\varepsilon \mid \# \tau=n\right)=0,
$$


where $c_{\mu, \mathrm{b}}$ is as in Theorem 1.1 and where $d_{\mathrm{Prok}}^{(n)}$ stands for the Prokhorov distance on the space of finite measures on $\left(\mathcal{R}_{\mathrm{b}}, \frac{1}{\sqrt{a_{n}}} d_{\mathrm{gr}}\right)$. It implies that the following limit holds jointly with (1.6):

$$
\left(\mathcal{R}_{\mathrm{b}}, \frac{1}{\sqrt{a_{n}}} d_{\mathrm{gr}}, \varnothing, \frac{1}{n} \mathbf{m}_{\text {count }}^{\mathrm{b}}\right) \text { under } \mathbf{P}(\cdot \mid \# \tau=n) \underset{n \rightarrow \infty}{\stackrel{\text { (law) }}{\longrightarrow}}\left(T_{H, W}, d_{H, W}, r_{H, W}, c_{\mu, \mathrm{b}} \cdot \mu_{H, W}\right) .
$$

Theorem 1.1 is the analogue of Proposition 5 and Theorem 7 in J-F. Le Gall and L. [24] established for $\mathbb{Z}^{d}$-valued BRWs. Let us mention that our strategy of proof is similar: we define a specific invariant shift for infinite tree-valued BRWs and we use the subadditive ergodic theorem; the constant $c_{\mu, \mathrm{b}}$ is interpreted as the probability that the invariant BRW visits its starting point only once.

The proof of Theorem 1.2 is distinct from that of Theorem 1.1. On one hand, it relies on general arguments on weak limits of random metrics (see Proposition 4.4). As an application of these results, we prove that the range of critical biased RWs on $N$-type supercritical GW-trees converges to the tree coded by a reflected Brownian motion (see Corollary 4.16). This result is derived from a much more difficult result due to A. Dembo and N. Sun [8] that asserts that the distance from the root of the RW converges, when suitably rescaled, to a reflected Brownian motion. The same idea allows to recover previous scaling limits for the range of RWs on supercritical GW-trees such as in D. [12] (critical biased RWs on b-ary trees) or in E. Aïdékon and L. de Raphélis [2] (biased RWs and RWs in random environment on single-type GW-trees). We refer to the end of Section 4.2 for more details. On the other hand, the proof of Theorem 1.2 uses limit theorems for discrete snakes that have been obtained by S. Janson and J-F. Marckert in [18] in the Brownian case and by C. Marzouk [29] in the stable cases.

\section{Organization of the paper}

The paper is organised as follows. In Section 2, we introduce notations on trees with an infinite line of ancestors that constitute a natural state-space for invariant tree-valued BRWs. In Section 3.1, we define the kinds of BRWs that we study; Section 3.2 is devoted to metric properties of the range of the so-called free BRWs. In Section 3.3, we state a coupling for BRWs that is a key argument in the proof of Theorem 1.1. In Section 3.4, we prove estimates that are used mostly to prove that $c_{\mu, \mathrm{b}}>0$. Theorem 1.1 is proved in Sections 3.5 and 3.6. In Section 4.1, we prove general convergence results for random metrics. In Section 4.2 we apply these results to get scaling limits for the range of RWs on $N$-type GW-trees (see Corollary 4.16). In Section 4.3 we introduce snake metrics and we prove specific results. In Sections 4.4 and 4.5, we recall definitions and properties on stable Lévy trees and Lévy snakes. Section 4.6 is devoted to basic properties of reflected Brownian cactuses. Theorem 1.2 is proved in Section 5.

\section{Tree with a possibly infinite line of ancestors}

Words. Recall that $\mathbb{N}$ stands for the set of nonnegative integers $\{0,1,2, \ldots\}$ and that $\mathbb{N}^{*}=\mathbb{N} \backslash\{0\}$. Let $A$ be a set with more than two elements that is viewed as an alphabet. We denote by $\mathbb{W}_{A}$ the finite words written with alphabet $A$ : namely,

$$
\mathrm{W}_{A}=\bigcup_{n \in \mathbb{N}} A^{n} .
$$

Here, $A^{0}$ is taken as $\{\varnothing\}, \varnothing$ being the empty word. Let $u=\left(a_{1}, \ldots, a_{n}\right) \in \mathbb{W}_{A}$ be distinct from $\varnothing$. We set $|u|=n$ that is the height of $u$, with the convention that $|\varnothing|=0$. We next set $\overleftarrow{u}=\left(a_{1}, \ldots, a_{n-1}\right)$ that is interpreted as the parent of $u$ (if $n=1$, then $\overleftarrow{u}=\varnothing$ ). More generally for all $p \in\{1, \ldots, n\}$, we set $u_{\mid p}=\left(a_{1}, \ldots, a_{p}\right)$, with the convention: $u_{\mid 0}=\varnothing$. For 
all $v=\left(b_{1}, \ldots, b_{m}\right) \in \mathbb{W}_{A}$, we set $u * v=\left(a_{1}, \ldots, a_{n}, b_{1}, \ldots, b_{m}\right)$ that is the concatenation of $u$ with $v$, with the convention that $\varnothing * u=u * \varnothing=u$. We shall also define the most recent common ancestor of $u$ and $v$ in $\mathbb{W}_{A}$ as $u \wedge v=u_{\mid p}=v_{\mid p}$ where $p=\max \left\{k \in \mathbb{N}: u_{\mid k}=v_{\mid k}\right\}$. We shall consider three cases:

- $A=\mathbb{N}^{*}$; in that case we use the notation $\mathbb{U}:=\mathbb{W}_{\mathbb{N}^{*}}$, the letter $U$ being for Ulam.

- $A=\{1, \ldots, \mathrm{b}\}, \mathrm{b}$ being an integer $\geq 2$; we use the notation $\mathbb{W}_{\mathrm{b}}:=\mathbb{W}_{\{1, \ldots, \mathrm{b}\}} ; \mathbb{W}_{\mathrm{b}}$ is the b-ary tree.

- $A=[0,1]$; we call $\mathbb{W}_{[0,1]}$ the free tree.

Definition 2.1. Rooted ordered trees can be viewed as subsets $t \subset \mathbb{U}$ that satisfy the following.

(a) $\varnothing \in t$.

(b) If $u \in t \backslash\{\varnothing\}$, then $\overleftarrow{u} \in t$

(c) For all $u \in t$, there exists $k_{u}(t) \in \mathbb{N}$ such that $u *(i) \in t$ if and only if $1 \leq i \leq k_{u}(t)$.

We denote by $\mathrm{T}$ the set of rooted ordered trees.

The quantity $k_{u}(t)$ is interpreted as the number of children of $u$ and $u *(i)$ is the $i$-th child of $u, 1 \leq i \leq k_{u}(t)$. If $k_{u}(t)=0$, then there is no child stemming from $u$ and assertion $(c)$ is empty. We next set the shift of $t$ at $u$ by $\theta_{u} t=\{v \in \mathbb{U}: u * v \in t\}$ that is also a rooted ordered tree: it is viewed as the subtree of the descendants stemming from $u$. Unless otherwise specified, all the random variables that are mentioned in this paper are defined on the same probability space $(\Omega, \mathcal{F}, \mathbf{P})$.

Definition 2.2. We equip $\mathbb{T}$ with the sigma-field $\mathscr{F}(\mathbb{T})$ generated by the sets $\{t \in \mathbb{T}: u \in t\}$, $u \in \mathbb{U}$. A Galton-Watson tree with offspring distribution $\mu$ (a $G W(\mu)$-tree, for short) is a $(\mathscr{F}, \mathscr{F}(\mathbb{T}))$-measurable r.v. $\tau: \Omega \rightarrow \mathbb{T}$ that satisfies the following.

- $k_{\varnothing}(\tau)$ has law $\mu$.

- For all $k \geq 1$ such that $\mu(k)>0$, the subtrees $\theta_{(1)} \tau, \ldots, \theta_{(k)} \tau$ under $\mathbf{P}\left(\cdot \mid k_{\varnothing}(\tau)=k\right)$ are independent with the same law as $\tau$ under $\mathbf{P}$.

Recall that $\tau$ is a.s. finite if and only if $\mu$ is critical or subcritical: $\sum_{k \geq 1} k \mu(k) \leq 1$.

Bilateral words. We shall consider branching random walks seen from the spatial and genealogical position of a tagged individual. To that end, it is convenient to introduce ordered trees that are rooted possibly at a negative generation and we also introduce their local limits that may have an infinite line of ancestors. It is therefore convenient to introduce words indexed by possibly negative numbers.

To simplify, we set $\mathbb{Z}_{-}=\mathbb{Z} \backslash \mathbb{N}^{*}$ and $\overleftarrow{\mathbb{Z}}=\mathbb{Z} \cup\{-\infty\}$. An A-bilateral word is a (possibly infinite) sequence $u=\left(a_{k}\right)_{k_{1}<k \leq k_{2}}$ where $k_{1} \in \overleftarrow{\mathbb{Z}}$ and $k_{2} \in \mathbb{Z}$ are such that $k_{2} \geq k_{1}$ and where $a_{k} \in A$ for all $k_{1}<k \leq k_{2}$. We denote by $\bar{W}_{A}$ the set of bilateral words, including the empty word denoted by $\varnothing$ (if $k_{1} \geq k_{2}$, then we agree on $u=\varnothing$ ). If $u \neq \varnothing$, we introduce the following notation.

$$
\begin{aligned}
& |u|_{-}=k_{1} \text {, be the depth of } u, \\
& |u|=k_{2}, \text { be the relative height of } u \text { (note that it may take negative values), } \\
& \overleftarrow{u}=\left(a_{k}\right)_{k_{1}<k \leq k_{2}-1} \text { be the parent of } u, \\
& u_{\left.\right|_{\left(l_{1}, l_{2}\right]}}=\left(a_{k}\right)_{k_{1} \vee l_{1}<k \leq k_{2} \wedge l_{2}}, \\
& \text { end }(u)=a_{k_{2}},
\end{aligned}
$$

for all $l_{1} \in \overleftarrow{\mathbb{Z}}, l_{2} \in \mathbb{Z}$ such that $l_{1} \leq l_{2}$. To simplify, we also set

$$
\forall l \in \mathbb{Z}, \quad u_{\mid l}=u_{\mid(-\infty, l]} .
$$


Let us stress that a bilateral word has at most a finite number of letters $a_{k}$ indexed by positive indices, $k \in \mathbb{N}^{*}$, while it can have infinitely many letters indexed by negative indices, $k \in \overleftarrow{\mathbb{Z}} \cap \mathbb{Z}_{-}$. Note that $\varnothing$ has neither relative height nor depth. Note that if $l_{1} \leq k_{1}$ and $k_{2} \leq l_{2}$, then $\left.u\right|_{\left(l_{1}, l_{2}\right]}=u$.

- Shift. For all $l \in \mathbb{Z}$, we denote the $l$-shift operator $\varphi_{l}: \overline{\mathbb{W}}_{A} \rightarrow \overline{\mathbb{W}}_{A}$ by:

$$
\varphi_{l}(u)=\left(a_{k+l}\right)_{k_{1}-l<k \leq k_{2}-l},
$$

Note that $\left|\varphi_{l}(u)\right|_{-}=|u|_{-}-l$, that $\left|\varphi_{l}(u)\right|=|u|-l$ and that $\varphi_{l}(\varnothing)=\varnothing$. Clearly, $\varphi_{l} \circ \varphi_{l^{\prime}}=\varphi_{l+l^{\prime}}$, $\varphi_{l}$ is bijective and $\varphi_{l} \circ \varphi_{-l}$ is the identity map.

- Concatenation. For all $u=\left(a_{k}\right)_{k_{1}<k \leq k_{2}} \in \overline{\mathrm{W}}_{A}$ and all $v=\left(b_{k}\right)_{1 \leq k \leq k_{3}} \in \mathbb{W}_{A}$, we define

$$
u * v=\left(c_{l}\right)_{k_{1}<k \leq k_{2}+k_{3}} \quad \text { where } \quad c_{k}= \begin{cases}a_{k} & \text { If } k_{1}<k \leq k_{2}, \\ b_{k-k_{2}} & \text { If } k_{2}<k \leq k_{2}+k_{3} .\end{cases}
$$

The bilateral word $u * v$ is the concatenation of a bilateral word $u$ on the left with a null depth word $v$ on the right. Note that $|u * v|_{-}=|u|_{-}$, that $|u * v|=|u|+|v|$ and that $u * \varnothing=u$. - Convergence in $\overline{\mathbb{W}}_{A}$. Assume that $\left(A, d_{A}\right)$ is a Polish space. We equip $\overline{\mathbb{W}}_{A}$ with the following local convergence.

Let $u^{(p)} \in \overline{\mathbb{W}}_{A}, p \in \mathbb{N}$; the sequence of words $u^{(p)}$ converges to $u$ if $\left|u^{(p)}\right|_{-} \rightarrow|u|_{-}$in $\overleftarrow{\mathbb{Z}}$ and if for all $l \in \mathbb{N}$ and for all $\varepsilon \in(0,1)$, there exists $p_{l, \varepsilon} \in \mathbb{N}$ such that for all $p \geq p_{l, \varepsilon}$, $\left|u^{(p)}\right|=|u|,(-l) \vee\left|u^{(p)}\right|_{-}=(-l) \vee|u|_{-}$and $\max _{(-l) \vee|u|_{-} \leq k \leq|u|} d_{A}\left(u_{k}^{(p)}, u_{k}\right)<\varepsilon$.

It is easy to see that this convergence corresponds to a Polish metric and we equip $\overline{\mathrm{W}}_{A}$ with the corresponding Borel sigma-field. Note that the shifts operators $\varphi_{l}$ are homeomorphisms with respect to local convergence. If $A=\mathbb{N}^{*}$, we shall use the notation $\overline{\mathbb{U}}:=\overline{\mathbb{W}}_{\mathbb{N}^{*}}$. If $A=\{1, \ldots, \mathrm{b}\}$, we shall use the notation $\overline{\mathrm{W}}_{\mathrm{b}}:=\overline{\mathbb{W}}_{\{1, \ldots, \mathrm{b}\}}$. Note that in these cases, $\mathbb{N}^{*}$ and $\{1, \ldots, \mathrm{b}\}$ are equipped with the discrete topology.

\section{Trees with infinite line of ancestors.}

Definition 2.3. A non-empty subset $R \subset \overline{\mathrm{W}}_{A}$ is a subtree of $\overline{\mathrm{W}}_{A}$ if it satisfies the following.

(a) There exists $|R|_{-} \in \overleftarrow{\mathbb{Z}}$ such that $|v|_{-}=|R|_{-}$for all $v \in R \backslash\{\varnothing\}$.

(b) For all $v \in R \backslash\{\varnothing\}$ such that $|v|>|R|_{-}, \overleftarrow{v} \in R$

(c) For all $u$ and $v$ in $R$, there exists $l \in \mathbb{Z}$ such that $u_{\mid l}=v_{\mid l}$ (see (2.3)).

We call $|R|_{-}$the depth of the subtree $R$. If $|R|_{-}>-\infty$, then (b) implies that the empty word $\varnothing$ is an element of $R$ and $|R|_{-}+1=\min _{v \in R \backslash\{\varnothing\}}|v|,(c)$ is always fulfilled since for all $l \leq|R|_{-}$and all $v \in R$, we get $v_{\mid l}=\varnothing$. If $|R|_{-}=-\infty$, then $\varnothing \notin R$.

- Common ancestor. Recall from (2.3) the notation $u_{\mid l}$. Let $R \subset \overline{\mathrm{W}}_{A}$ be a subtree as in Definition 2.3. We define the common ancestor of $u, v \in R$ by

$$
u \wedge v=u_{\mid b(u, v)}=v_{\mid b(u, v)} \quad \text { where } \quad b(u, v):=\max \left\{l \in \mathbb{Z}: u_{\mid l}=v_{\mid l}\right\},
$$

that is well-defined thanks to Definition $2.3(c)$.

- Graph distance on subtrees of $\overline{\mathrm{W}}_{A}$. A subtree $R \subset \overline{\mathrm{W}}_{A}$ as in Definition 2.3 corresponds to the following graph-tree: its set of vertices is $R$ and its set of edges is $\{\{v, \overleftarrow{v}\} ; v \in R$ : $\left.|v|>|R|_{-}\right\}$. We easily observe that the graph distance $d_{\mathrm{gr}}$ on $R$ is given by

$$
\forall x, y \in R, \quad d_{\mathrm{gr}}(x, y)=|x|+|y|-2|x \wedge y| .
$$

- Ordered trees with a possibly infinite line of ancestors. We next extend Definition 2.1 to ordered trees with a possibly infinite line of ancestors as follows. 
Definition 2.4. Recall the notation $\overline{\mathbb{U}}:=\overline{\mathbb{W}}_{\mathbb{N}^{*}}$. A subset $t \subset \overline{\mathbb{U}}$ is an ordered tree (with a possibly infinite line of ancestors) if it is a subtree of $\overline{\mathbb{U}}$ as in Definition 2.3 satisfying $(a)$, (b) and (c) with $A=\mathbb{N}^{*}$ and if it furthermore satisfies the condition that any word has a finite number of children, that is,

(d) $\forall u \in t, k_{u}(t):=\#\{v \in t: \overleftarrow{v}=u\}<\infty$ and $\left\{1, \ldots, k_{u}(t)\right\}=\{\operatorname{end}(v) ; v \in t: \overleftarrow{v}=u\}$ if $k_{u}(t) \geq 1$ We shall consider that the singleton $\{\varnothing\}$ is the only tree with one point. We denote by $\overline{\mathbb{T}}$ the set of ordered trees.

For all $k \in \overleftarrow{\mathbb{Z}}$, we set $\mathbb{T}_{k}=\{\{\varnothing\}\} \cup\left\{t \in \overline{\mathbb{T}}:|t|_{-}=k\right\}$. Note that $\mathbb{T}_{0}=\mathbb{T}$, where $\mathbb{T}$ is as in Definition 2.1 and observe that $\varphi_{-k}(\mathbb{T})=\mathbb{T}_{k}$ when $k>-\infty$.

- Lexicographical order and successor of a vertex. Let $t \in \overline{\mathrm{T}}$. By Definition $2.3(c)$, the vertices of $t$ are totally ordered by the lexicographical order $\leq_{t}$ that is formally defined as follows. Let $u, v \in t \backslash\{\varnothing\}$; recall from (2.5) the definition of $b(u, v)$ and from (2.2) the definition of end $(\cdot)$; then,

$$
u \leq_{t} v \text { if and only if } \operatorname{end}\left(u_{\mid b(u, v)+1}\right) \leq \operatorname{end}\left(v_{\mid b(u, v)+1}\right) .
$$

Note that $\leq_{t}$ actually depends on $t$ : it is not defined on the whole set of bilateral words $\overline{\mathbb{U}}$ but only on $t$ (indeed, to define $\leq_{t}$, branching points have to be well-defined, which requires possibly infinite words to share a prefix). If $\varnothing \in t$, then $\varnothing$ is the $\leq_{t}$-least element of $t$. We denote by $<_{t}$ the strict order associated with $\leq_{t}$. We also introduce the following related notation: for all $u \in t$, the successor $\operatorname{scc}(u)$ of $u$ is defined as the $\leq_{t}$-least element of $\left\{v \in t: u<_{t} v\right\}$ if this set is not empty, otherwise we $\operatorname{simply}$ take $\operatorname{scc}(u)=u$.

- Subtree. Let $t \in \overline{\mathrm{T}}$ and $u \in t$. The subtree $\theta_{u} t$ stemming from $u$ is defined as follows.

$$
\theta_{u} t=\{v \in \mathbb{U}: u * v \in t\}
$$

where we recall from (2.4) the definition of the concatenation $*$ of a bilateral word on the left with a null-depth word on the right. Note that $\left|\theta_{u} t\right|_{-}=0$, namely: $\theta_{u} t \in \mathbb{T}$, where $\mathrm{T}$ is as in Definition 2.1.

Pointed labelled trees. To deal with branching random walks, we introduce labelled trees where the label of a vertex is viewed as its position in space. More precisely, let $\left(E, d_{E}\right)$ be a Polish metric space. We define the space of pointed E-labelled trees as follows. For all $k \in \overleftarrow{\mathbb{Z}}$, we set:

$$
\mathbb{T}_{k}^{\bullet}(E)=\{\partial\} \cup\left\{\mathbf{t}=\left(t, \varrho ; \mathbf{x}=\left(x_{v}\right)_{v \in t}\right): t \in \mathbb{T}_{k}, \varrho \in t, x_{v} \in E, v \in t\right\} \quad \text { and } \quad \overline{\mathbb{T}}(E)=\bigcup_{k \in \overleftarrow{\mathbb{Z}}} \mathbb{T}_{k}^{\bullet}(E)
$$

where $\partial$ stands for a cemetery point. Here, the label of $v \in t$ is $x_{v} \in E$ that is viewed as the spatial position of $v$. If there is no label, we simply write $\mathrm{T}_{k}^{\bullet}$ and $\overline{\mathrm{T}}^{\bullet}$.

- Shift operator on labelled trees. Shift operators act naturally on the space of pointed $E$-labelled trees as follows: let $l \in \mathbb{Z}$; we set $\varphi_{l}(\partial)=\partial$ and for all $\mathbf{t}=(t, \varrho ; \mathbf{x}) \in \overline{\mathbb{T}}^{\bullet}(E) \backslash\{\partial\}$, we set

$$
\varphi_{l}(\mathbf{t})=\left(\varphi_{l}(t), \varphi_{l}(\varrho) ; \mathbf{x} \circ \varphi_{-l}\right), \quad \text { where } \quad \mathbf{x} \circ \varphi_{-l}=\left(x_{\varphi_{-l}(w)}\right)_{w \in \varphi_{l}(t)} .
$$

- Truncation. We next define a natural truncation procedure for pointed labelled trees along the line of ancestors of the distinguished point.

Definition 2.5. Let $\mathbf{t}=\left(t, \varrho ; \mathbf{x}=\left(x_{v}\right)_{v \in t}\right) \in \overline{\mathbb{T}}^{\bullet}(E)$. Let $p \in \overleftarrow{\mathbb{Z}}$ and $q \in \mathbb{Z} \cup\{\infty\}$ be such that $p<q$. We define the following.

- If $(p, q] \cap\left(|\varrho|_{-},|\varrho|\right]=\emptyset$, then we set $[\mathbf{t}]_{p}^{q}=\partial$. We also set $[\partial]_{p}^{q}=\partial$. 
- If $(p, q] \cap\left(|\varrho|_{-},|\varrho|\right] \neq \emptyset$, then we set $[\mathbf{t}]_{p}^{q}=\left(t^{\prime}, \varrho^{\prime} ; \mathbf{x}^{\prime}\right)$ where

$$
\varrho^{\prime}=\varrho_{\mid(p, q]}, \quad t^{\prime}=\left\{v_{\mid(p, q]} ; v \in t: v_{\mid p}=\varrho_{\mid p}\right\} \text { and } x_{v^{\prime}}^{\prime}=x_{v},
$$

where $v \in t$ is such that $v^{\prime}=v_{\mid(p, q]}$ and $v_{\mid p}=\varrho_{\mid p}$ (recall notation $v_{\mid p}$ from (2.3)).

We simply set $[\mathbf{t}]_{p}$ instead of $[\mathbf{t}]_{p}^{\infty}$. If $\mathbf{t} \in \mathbb{T}_{k}^{\bullet}(E)$, then note that $[\mathbf{t}]_{p}^{q} \in \mathbb{T}_{k \vee p}^{\bullet}(E)$. We use a similar notation for pointed trees without label.

- Local convergence on $\overline{\mathbb{T}}^{\bullet}(E)$. For all $\mathbf{t}=(t, \varrho ; \mathbf{x}), \mathbf{t}^{\prime}=\left(t^{\prime}, \varrho^{\prime} ; \mathbf{x}^{\prime}\right)$ in $\overline{\mathbb{T}}^{\bullet}(E)$, we first set

$$
\Delta\left(\mathbf{t}, \mathbf{t}^{\prime}\right)=\mathbb{1}_{\left\{(t, \varrho) \neq\left(t^{\prime}, \varrho^{\prime}\right)\right\}}+\mathbb{1}_{\left\{(t, \varrho)=\left(t^{\prime}, \varrho^{\prime}\right)\right\}} \max _{v \in t}\left(1 \wedge d_{E}\left(x_{v}, x_{v}^{\prime}\right)\right)
$$

with $\Delta(\mathbf{t}, \partial)=1$ and $\Delta(\partial, \partial)=0$. We easily check that $\Delta$ is a metric on $\overline{\mathbb{T}}^{\bullet}(E)$. Then, we define the local convergence as follows.

Let $\mathbf{t}_{n}=\left(t^{(n)}, \varrho^{(n)} ; \mathbf{x}^{(n)}\right) \in \overline{\mathbb{T}}^{\bullet}(E), n \in \mathbb{N}$; the sequence $\left(\mathbf{t}_{n}\right)_{n \geq 0}$ is said to converge locally to $\mathbf{t}=(t, \varrho ; \mathbf{x}) \in \overline{\mathbb{T}}^{\bullet}(E)$ if for all $\varepsilon \in(0,1)$ and all $q \in \mathbb{N}$, there exists $n_{q, \varepsilon} \in \mathbb{N}$ such that for all integers $n \geq n_{q, \varepsilon}, \Delta\left(\left[\mathbf{t}^{(n)}\right]_{-q}^{q},[\mathbf{t}]_{-q}^{q}\right)<\varepsilon$ (when there is no label, it simply means that $\left[\mathbf{t}^{(n)}\right]_{-q}^{q}=[\mathbf{t}]_{-q}^{q}$ ).

Local convergence corresponds for instance to the following metric:

$$
\forall \mathbf{t}, \mathbf{t}^{\prime} \in \mathbb{T}^{\bullet}(E), \quad \boldsymbol{\delta}_{1 \mathrm{oc}}\left(\mathbf{t}, \mathbf{t}^{\prime}\right)=\sum_{q \in \mathbb{N}} 2^{-q-1} \Delta\left([\mathbf{t}]_{-q}^{q},\left[\mathbf{t}^{\prime}\right]_{-q}^{q}\right),
$$

with $\boldsymbol{\delta}_{\text {loc }}(\mathbf{t}, \partial)=1$ and $\boldsymbol{\delta}_{\text {loc }}(\partial, \partial)=0$. We easily check that $\left(\mathbb{T}^{\bullet}(E), \boldsymbol{\delta}_{\text {loc }}\right)$ is Polish and we note that shift operators are isometries.

Definition 2.6. Let $(t, \varrho) \in \overline{\mathbb{T}}^{\bullet}$ be distinct from $\partial$.

(a) We set cent $(t, \varrho)=\varphi_{|\varrho|}(t, \varrho)$ that is the centering map: it shifts trees so that their distinguished point is at relative height 0.

(b) We next $\operatorname{set} \operatorname{scc}(t, \varrho)=\operatorname{cent}(t, \operatorname{scc}(\varrho))$ where we recall that $\operatorname{scc}(\varrho)$ stands for the vertex of $t$ coming next in the lexicographical order as defined by (2.7). We call $\operatorname{scc}(\cdot)$ the successor map.

(c) Observe that there is a unique pointed tree $\left(t^{\prime}, \varrho^{\prime}\right) \in \overline{\mathrm{T}}^{\bullet}$ and a unique one-to-one map $\psi: t^{\prime} \rightarrow\left\{\varrho_{\mid p} ; p \leq|\varrho|\right\} \cup\left\{v \in t: \varrho<_{t} v\right\}$ such that $\psi\left(\varrho^{\prime}\right)=\varrho$, that is increasing with respect to the lexicographical order and that preserves the relative height; we set $[(t, \varrho)]^{+}=\left(t^{\prime}, \varrho^{\prime}\right)$ that is called the right-part of $(t, \varrho)$. Intuitively, the difference between $\left(t^{\prime}, \rho^{\prime}\right)$ and $\left\{\varrho_{\mid p} ; p \leq|\varrho|\right\} \cup\left\{v \in t: \varrho<_{t} v\right\}$ is that $\left(t^{\prime}, \rho^{\prime}\right)$ respects the convention (c) in Definition 2.1 we have imposed on rooted ordered trees. We also set

$$
\operatorname{scc}^{+}(t, \varrho)=[\operatorname{scc}(t, \varrho)]^{+} .
$$

The map $\mathrm{scc}^{+}(\cdot)$ is called the right-successor.

By convenience, we set $\operatorname{cent}(\partial)=\operatorname{scc}(\partial)=\operatorname{scc}^{+}(\partial)=\partial$.

Note that

$$
[\operatorname{cent}(t, \varrho)]^{+}=\operatorname{cent}\left([(t, \varrho)]^{+}\right) \quad \text { and } \operatorname{scc}^{+}(t, \varrho)=\operatorname{scc}^{+}\left([(t, \varrho)]^{+}\right) .
$$

Let us state a technical result about the continuity of the maps cent, $[\cdot]^{+}, \mathrm{scc}$ and $\mathrm{scc}^{+}$. Lemma 2.7. The maps cent $(\cdot)$ and $[\cdot]^{+}$are locally continuous and the maps $\operatorname{scc}(\cdot)$ and $\operatorname{scc}^{+}(\cdot)$ are locally continuous at the pointed trees $(t, \varrho) \operatorname{such}$ that $\operatorname{scc}(\varrho) \neq \varrho$.

Proof. Since shift-operators are $\boldsymbol{\delta}_{1 \mathrm{oc}}$-isometries, the continuity of cent follows from the continuity of $(t, \varrho) \rightarrow|\varrho|$ that is a direct consequence of the definition of local convergence; $[\cdot]^{+}$is locally continuous because $\left[[\mathbf{t}]_{-q}^{q}\right]^{+}=\left[[\mathbf{t}]^{+}\right]_{-q}^{q}$ and to complete the 
proof, it is then sufficient to prove that $(t, \varrho) \mapsto(t, \operatorname{scc}(\varrho))$ is locally continuous at trees such that $\operatorname{scc}(\varrho) \neq \varrho$.

To that end, let $\left(t_{n}, \varrho_{n}\right) \rightarrow(t, \varrho)$ locally in $\overline{\mathbb{T}}^{\bullet}$. Set $p_{0}=|\operatorname{scc}(\varrho)|$ and suppose that $\varrho \neq \operatorname{scc}(\varrho)$, which implies $|\varrho \wedge \operatorname{scc}(\varrho)|=p_{0}-1$. Let $p \in \mathbb{N}$ be such that $-p<p_{0}-1<|\varrho|+1<p$; there is $n_{p} \in \mathbb{N}$ such that for all $n \geq n_{p},\left(t_{n}^{\prime}, \varrho_{n}^{\prime}\right):=\left[\left(t_{n}, \varrho_{n}\right)\right]_{-p}^{p}$ is equal to $\left(t^{\prime}, \varrho^{\prime}\right):=[(t, \varrho)]_{-p}^{p}$; thus, the successor of $\varrho_{n}^{\prime}$ in $t_{n}^{\prime}$ is equal to the successor of $\varrho^{\prime}$ in $t^{\prime}$. Consequently, $\left[\left(t_{n}, \operatorname{scc}\left(\varrho_{n}\right)\right)\right]_{-p}^{p}=\left(t_{n}^{\prime}, \operatorname{scc}\left(\varrho_{n}^{\prime}\right)\right)=\left(t^{\prime}, \operatorname{scc}\left(\varrho^{\prime}\right)\right)=[(t, \operatorname{scc}(\varrho))]_{-p}^{p}$, which entails the desired result.

Infinite pointed Galton-Watson trees. For all $t \in \overline{\mathbb{T}}$ and for all $u \in t$, recall from (2.8) the definition of the subtree $\theta_{u} t \in \mathbb{T}$.

Definition 2.8. Let $(r(j, k))_{k \geq j \geq 1}$ be a probability measure on the octant $\left\{(j, k) \in\left(\mathbb{N}^{*}\right)^{2}\right.$ : $j \leq k\}$. Let $\mu$ be a probability measure on $\mathbb{N}$. Let $\tau^{*}=\left(\tau^{*}, \varrho\right): \Omega \rightarrow \overline{\mathbb{T}}^{\bullet}$ be a Borel-measurable random pointed tree such that a.s. $|\varrho|=0$ and $\left|\tau^{*}\right|_{-}=-\infty$. We introduce the following notation.

$$
\forall p \in \mathbb{N}, \quad \varrho(p)=\varrho_{\mid]-\infty,-p]}, \quad \operatorname{Sp}=\{\varrho(p) ; p \in \mathbb{N}\} \text { and } \partial \mathrm{Sp}=\left\{u \in \tau^{*} \backslash \mathrm{Sp}: \overleftarrow{u} \in \mathrm{Sp}\right\}
$$

Then, $\tau^{*}$ is an infinite pointed Galton-Watson tree with offspring distribution $\mu$ and dispatching measure $r$ if the r.v. $S:=\left(\operatorname{end}(\varrho(p)), k_{\varrho(p+1)}\left(\tau^{*}\right)\right)_{p \in \mathbb{N}}$ are i.i.d. with law $r$ and if conditionally given $S$, the subtrees $\left(\theta_{u} \tau ; u \in \partial \mathrm{Sp}\right)$ are independent $G W(\mu)$-trees.

We shall deal with the following special cases that are well-defined if $m_{\mu}:=\sum_{k \in \mathbb{N}} k \mu(k)$ is a finite quantity.

(i) If $r(j, k)=\mu(k) / m_{\mu}$, for all $k \geq j \geq 1$, then we say that $\tau^{*}$ is an infinite pointed $G W(\mu)$-tree (an IPGW( $\mu$ )-tree for short).

(ii) If $r(j, k)=\mathbb{1}_{\{j=1\}} \bar{\mu}(k)$, for all $k \geq j \geq 1$, where $\bar{\mu}(k)=m_{\mu}^{-1} \sum_{l \geq k} \mu(l)$ for all $k \geq 1$, then we say that $\tau^{*}$ is the right part of an infinite pointed $G W(\mu)$-tree (an IPGW $W^{+}(\mu$ )-tree for short).

Note that if $\tau^{*}$ is an IPGW $(\mu)$-tree, then $\left[\tau^{*}\right]^{+}$is an IPGW' $(\mu)$-tree.

IPGW-trees are related to GW-trees via the many-to-one principle (or the one-point decomposition of $G W$-trees) that asserts the following: let $\mu$ be a probability distribution on $\mathbb{N}$ such that $m_{\mu}:=\sum_{k \in \mathbb{N}} k \mu(k)<\infty$. Let $\tau$ be a GW( $\mu$ )-tree and let $\tau^{*}$ be an IPGW $(\mu)$ tree as in Definition 2.8. Then for all Borel-measurable functions $F: \mathbb{N} \times \overline{\mathbb{T}}^{\bullet} \rightarrow[0, \infty)$,

$$
\mathbf{E}\left[\sum_{v \in \tau} F\left(|v| ; \varphi_{|v|}(\tau, v)\right)\right]=\sum_{p \geq 0} m_{\mu}^{p} \mathbf{E}\left[F\left(p ;\left[\boldsymbol{\tau}^{*}\right]_{-p}\right)\right]
$$

where we recall from Definition 2.5 the notation $\left[\tau^{*}\right]_{-p}$ for the pointed tree $\tau^{*}$ truncated above the ancestor of $\varrho$ at generation $-p$. Based on this identity, the following proposition shows that IPGW trees are local limits of critical GW-trees conditioned to be large and seen from a uniformly chosen vertex. This result is part of the folklore; its proof derives from (2.10) and it is left to the reader.

Proposition 2.9. Let $\mu$ be a probability distribution on $\mathbb{N}$ such that $\sum_{k \in \mathbb{N}} k \mu(k)=1$. We assume that $\mu$ is aperiodic. Let $\tau$ be a $G W(\mu)$-tree; let $\mathbf{u}$ be uniformly distributed on the set of vertices of $\tau$. Let $\tau^{*}$ be an IPGW $(\mu)$-tree as in Definition 2.8. Then

$$
\operatorname{cent}(\tau, \mathbf{u}) \text { under } \mathbf{P}(\cdot \mid \# \tau=n) \underset{n \rightarrow \infty}{\longrightarrow} \tau^{*}
$$

weakly on $\overline{\mathrm{T}}^{\bullet}$ with respect to local convergence. 
We use the previous proposition to prove the following one.

Proposition 2.10. Let $\mu$ be a probability measure on $\mathbb{N}$ such that $\sum_{k \in \mathbb{N}} k \mu(k)=1$. Recall $\operatorname{scc}(\cdot)$ and $\operatorname{scc}^{+}(\cdot)$ from Definition 2.6. Then, the law of IPGW( $\mu$ )-trees (resp. IPGW $W^{+}(\mu)-$ trees) is preserved by $\operatorname{scc}(\cdot)$ (resp. by $\operatorname{scc}^{+}(\cdot)$ ).

Proof. Let us first mention that a different proof of the result for $\operatorname{scc}^{+}(\cdot)$ is given in Proposition 2 in Le Gall and L. [24]. Then, note that the result for $\mathrm{scc}^{+}(\cdot)$ is implied by the result for $\operatorname{scc}(\cdot)$ by (2.9) and since the right-part of a IPGW $(\mu)$-tree is an $\operatorname{IPGW}^{+}(\mu)$ tree. Let us prove the result for $\operatorname{scc}(\cdot)$. Let $\tau^{*}=\left(\tau^{*}, \varrho\right)$ be an IPGW $(\mu)$-tree and let $\tau$ be a GW $(\mu)$-tree; let $\mathbf{u}$ be uniformly distributed on the set of vertices of $\tau$; denote by $v_{*}$ the last vertex of $\tau$ with respect to the lexicographical order. Set $\mathbf{u}^{\prime}=\operatorname{scc}(\mathbf{u})$ if $\mathbf{u} \neq v_{*}$ and $\mathbf{u}^{\prime}=\varnothing$ if $\mathbf{u}=v_{*}$ : clearly, $\mathbf{u}^{\prime}$ is uniformly distributed on $\tau$ and $\mathbf{P}\left(\operatorname{scc}(\mathbf{u}) \neq \mathbf{u}^{\prime} \mid \# \tau=n\right)=1 / n$. Let $F: \overline{\mathbb{T}}^{\bullet} \rightarrow[0, \infty)$ be locally continuous and bounded. By Definition $2.6(b), \operatorname{scc}(\tau, \mathbf{u})=$ $\operatorname{cent}(\tau, \operatorname{scc}(\mathbf{u}))$. Thus,

$$
\left|\mathbf{E}[F(\operatorname{scc}(\tau, \mathbf{u})) \mid \# \tau=n]-\mathbf{E}\left[F\left(\operatorname{cent}\left(\tau, \mathbf{u}^{\prime}\right)\right) \mid \# \tau=n\right]\right| \leq 2\|F\|_{\infty} / n .
$$

First suppose that $\mu$ is aperiodic. Then the previous inequality and Proposition 2.9 imply that

$$
\lim _{n \rightarrow \infty} \mathbf{E}[F(\operatorname{scc}(\tau, \mathbf{u})) \mid \# \tau=n]=\lim _{n \rightarrow \infty} \mathbf{E}\left[F\left(\operatorname{cent}\left(\tau, \mathbf{u}^{\prime}\right)\right) \mid \# \tau=n\right]=\mathbf{E}\left[F\left(\boldsymbol{\tau}^{*}\right)\right] .
$$

Moreover, since it is clear that a.s. $\operatorname{scc}(\varrho) \neq \varrho$, Lemma 2.7 and Proposition 2.9 entail

$$
\lim _{n \rightarrow \infty} \mathbf{E}[F(\operatorname{scc}(\tau, \mathbf{u})) \mid \# \tau=n]=\mathbf{E}\left[F\left(\operatorname{scc}\left(\boldsymbol{\tau}^{*}\right)\right)\right],
$$

which completes the proof when $\mu$ is aperiodic.

Let us consider a general $\mu$. For all $\varepsilon \in(0,1)$, we set $\mu_{\varepsilon}:=\varepsilon \delta_{1}+(1-\varepsilon) \mu$. Namely, $\mu_{\varepsilon}$ is a critical aperiodic offspring distribution. Let $\tau_{\varepsilon}^{*}$ be an infinite GW $\left(\mu_{\varepsilon}\right)$-tree. We easily check that $\boldsymbol{\tau}_{\varepsilon}^{*} \rightarrow \boldsymbol{\tau}^{*}$ locally as $\varepsilon \rightarrow 0$. The local continuity of $\operatorname{scc}(\cdot)$ (Lemma 2.7) entails the desired result.

\section{Tree-valued branching random walks}

\subsection{Definitions}

Let $E$ be a (Polish) space of labels. Let $\left(q\left(y, d y^{\prime}\right)\right)_{y \in E}$ be a transition kernel and let $\varpi$ be a Borel probability measure on $E$. For all pointed tree $\mathbf{t}=(t, u) \in \overline{\mathbb{T}}^{\bullet}$, we define the law $Q_{\varpi, \mathbf{t}}$ on $\overline{\mathbb{T}}^{\bullet}(E)$ of the $q$-branching random walk with genealogical tree $t$ and such that $\varpi$ is the law of the spatial position of the distinguished individual $u$.

To that end, we first assume that $t$ is finite and we introduce the following notation: for any $v, w \in t$, we denote by $\llbracket v, w \rrbracket$ the set of vertices on the shortest path joining $v$ to $w$ in the tree $t$. If $w \neq v$, then we denote by $\overleftarrow{w}^{v}$ the unique $v^{\prime} \in \llbracket v, w \rrbracket$ that is at graph-distance 1 from $w$; we call $\overleftarrow{w}^{v}$ the $v$-parent of $w$

Then, the r.v. $\boldsymbol{\Theta}=\left(t, u ;\left(Y_{v}\right)_{v \in \tau}\right): \Omega \rightarrow \overline{\mathbb{T}}^{\bullet}(E)$ has law $Q_{\varpi, \mathrm{t}}$ if

$$
\text { the joint law of }\left(Y_{v}\right)_{v \in t} \text { is } \varpi\left(d y_{u}\right) \prod_{v \in t \backslash\{u\}} q\left(y_{\overleftarrow{v}^{u}}, d y_{v}\right),
$$

The definition (3.1) can be extended to the case in which $\mathbf{t}$ is infinite. For such purpose it is enough to note that $[\Theta]_{p}^{q}$ has law $Q_{\varpi,[(t, \varrho)]_{p}^{q}}$ (see Definition 2.5 for the truncation []$_{p}^{q}$ ).

Let us note that

$$
\mathbf{t}_{n} \underset{n \rightarrow \infty}{\longrightarrow} \mathbf{t} \text { locally in } \overline{\mathrm{T}}^{\bullet} \Longrightarrow Q_{\varpi, \mathbf{t}_{n}} \underset{n \rightarrow \infty}{\longrightarrow} Q_{\varpi, \mathbf{t}} \text { weakly on } \overline{\mathrm{T}}^{\bullet}(E) .
$$


When $\varpi=\delta_{y}$ for some $y \in E$, we simply write $Q_{y, \mathbf{t}}$ instead of $Q_{\delta_{y}, \mathbf{t}}$. Since $y \mapsto q(y, A)$ is Borel-measurable for all Borel subsets $A$ of $E$, it is easy to check that $y \mapsto Q_{y, \mathrm{t}}$ is also Borel measurable and that $Q_{\varpi, \mathbf{t}}=\int_{E} \varpi(d y) Q_{y, \mathbf{t}}$.

As an immediate consequence of the definition, we also get the following: fix $l \in \mathbb{Z}$ and $\operatorname{set}\left(t^{\prime}, u^{\prime}\right)=\left(\varphi_{l}(t), \varphi_{l}(u)\right)$.

$$
\text { If } \boldsymbol{\Theta}=\left(t, u ;\left(Y_{v}\right)_{v \in t}\right) \text { has law } Q_{\varpi,(t, u)} \text {, then }\left(t^{\prime}, u^{\prime} ;\left(Y_{\varphi_{-l}(w)}\right)_{w \in t^{\prime}}\right) \text { has law } Q_{\varpi,\left(t^{\prime}, u^{\prime}\right)} \text {. }
$$

Definition 3.1. We fix $\mathbf{t}=(t, u) \in \overline{\mathbb{T}}^{\bullet}$. We shall consider mostly the four following cases.

(i) $E=\mathbb{W}_{\mathrm{b}}$, the b-ary tree equipped with the local convergence; $\varpi=\delta_{\varnothing}$ and $q(x, d y)=$ $p_{\mathrm{b}}^{+}(x, d y)$ where for all measurable $f: \mathbb{W}_{\mathrm{b}} \rightarrow[0, \infty)$,

$$
\int_{\mathbb{W}_{\mathrm{b}}} p_{\mathrm{b}}^{+}(x, d y) f(y)= \begin{cases}\frac{1}{2} f(\overleftarrow{x})+\frac{1}{2 \mathrm{~b}} \sum_{1 \leq i \leq \mathrm{b}} f(x *(i)) & \text { if } x \neq \varnothing \\ \frac{1}{\mathrm{~b}} \sum_{1 \leq i \leq \mathrm{b}} f((i)) & \text { if } x=\varnothing\end{cases}
$$

We denote by $Q_{\mathrm{t}}^{+\mathrm{b}}$ the law of the $\mathrm{W}_{\mathrm{b}}$-valued branching random walk with transition kernel $p_{\mathrm{b}}^{+}$and with "initial" position $\varnothing$ in $\mathbb{W}_{\mathrm{b}}$.

(ii) $E=\mathbb{W}_{[0,1]}$, the free tree equipped with the local convergence; $\varpi=\delta_{\varnothing}$ and $q(x, d y)=$ $p^{+}(x, d y)$ where for all measurable $f: \mathbb{W}_{[0,1]} \rightarrow[0, \infty)$,

$$
\int_{\mathrm{W}_{[0,1]}} p^{+}(x, d y) f(y)= \begin{cases}\frac{1}{2} f(\overleftarrow{x})+\frac{1}{2} \int_{0}^{1} d s f(x *(s)) & \text { if } x \neq \varnothing \\ \int_{0}^{1} d s f((s)) & \text { if } x=\varnothing .\end{cases}
$$

We denote by $Q_{\mathrm{t}}^{+}$the law of the $\mathbb{W}_{[0,1]}$-valued branching random walk with transition kernel $p^{+}$and with "initial" position $\varnothing$ in $\mathbb{W}_{[0,1]}$.

(iii) $E=\mathbb{W}_{\mathrm{b}}^{*}:=\left\{x \in \bar{W}_{\mathrm{b}}:|x|_{-}=-\infty\right\}$, equipped with the local convergence; we fix $\mathrm{o} \in \mathbb{W}_{\mathrm{b}}^{*}$ and we take $\varpi=\delta_{\mathrm{o}}$ and $q(x, d y)=p_{\mathrm{b}}(x, d y)$ where for all $x \in \mathbb{W}_{\mathrm{b}}^{*}$ and for all measurable $f: \mathbb{W}_{\mathrm{b}}^{*} \rightarrow[0, \infty)$,

$$
\int_{\mathbb{W}_{\mathrm{b}}^{*}} p_{\mathrm{b}}(x, d y) f(y)=\frac{1}{2} f(\overleftarrow{x})+\frac{1}{2 \mathrm{~b}} \sum_{1 \leq i \leq \mathrm{b}} f(x *(i))
$$

We denote by $Q_{\mathrm{o}, \mathrm{t}}^{\mathrm{b}}$ the law of the $\mathrm{W}_{\mathrm{b}}^{*}$-valued branching random walk with transition kernel $p_{\mathrm{b}}$ and with "initial" position o in $\mathrm{W}_{\mathrm{b}}^{*}$.

(iv) $E=\mathbb{W}_{[0,1]}^{*}:=\left\{x \in \overline{\mathbb{W}}_{[0,1]}:|x|_{-}=-\infty\right\}$, equipped with the local convergence; we fix $\mathrm{o} \in \mathbb{W}_{[0,1]}^{*}$; we take $\varpi=\delta_{\circ}$ and $q(x, d y)=p(x, d y)$, where for all $x \in \overline{\mathrm{W}}_{[0,1]}$ and for all measurable $f: \mathbb{W}_{[0,1]}^{*} \rightarrow[0, \infty)$,

$$
\int_{\mathbb{W}_{[0,1]}^{*}} p(x, d y) f(y)=\frac{1}{2} f(\overleftarrow{x})+\frac{1}{2} \int_{0}^{1} d s f(x *(s)) .
$$

We denote by $Q_{\mathrm{o}, \mathrm{t}}$ the law of the $\mathbb{W}_{[0,1]}^{*}$-valued branching random walk with transition kernel $p$ and with "initial" position o in $\mathbb{W}_{[0,1]}^{*}$ : we shall refer to this branching random walk as the free branching random walk.

Remark 3.2. Note that if $\boldsymbol{\Theta}=\left(t, u ;\left(X_{v}\right)_{v \in t}\right.$ ) has law $Q_{\mathrm{o}, \mathrm{t}}^{\mathrm{b}}$ (or $Q_{\mathrm{o}, \mathrm{t}}$ ) then $|\boldsymbol{\Theta}|:=(t, u$; $\left.\left(\left|X_{v}\right|\right)_{v \in t}\right)$ is a $\mathbb{Z}$-valued branching random walk whose spatial motion is that of the simple symmetric random walk. Similarly, if $\Theta$ has law $Q_{\mathrm{t}}^{+\mathrm{b}}$ (or $Q_{\mathrm{t}}^{+}$), then $|\Theta|$ is an $\mathbb{N}$-valued branching random walk whose spatial motion is that of the simple symmetric random walk reflected at 0 .

Definition 3.3. We define the b-contraction map $\Phi_{\mathrm{b}}: \overline{\mathrm{W}}_{[0,1]} \rightarrow \overline{\mathrm{W}}_{\mathrm{b}}$ as follows. For all $r \in(0, \infty)$, we set $\lceil r\rceil=\min \{k \in \mathbb{Z}: r \leq k\}$ and by convenience we take $\lceil 0\rceil=1$. Then, for all $x=\left(a_{k}\right)_{|x|_{-}<k \leq|x|} \in \overline{\mathbb{W}}_{[0,1]}$, we define

$$
\left.\Phi_{\mathrm{b}}(x)=\left(\left\lceil\mathrm{b} a_{k}\right\rceil\right)\right)_{|x|_{-}<k \leq|x|} \in \overline{\mathrm{W}}_{\mathrm{b}} .
$$


The b-contraction map is measurable and preserves the depth and the relative height of words.

Remark 3.4. Note that $\Phi_{\mathrm{b}}$ transforms respectively the kernel $p(x, d y)$ into $p_{\mathrm{b}}(x, d y)$ and the kernel $p^{+}(x, d y)$ into $p_{\mathrm{b}}^{+}(x, d y)$. It naturally extends to $\overline{\mathrm{W}}_{[0,1]}$-labelled pointed trees as follows: if $\Theta=\left(t, \varrho ;\left(x_{v}\right)_{v \in t}\right) \in \overline{\mathbb{T}}^{\bullet}\left(\overline{\mathbb{W}}_{[0,1]}\right)$, we set $\Phi_{\mathrm{b}}(\Theta)=\left(t, \varrho ;\left(\Phi_{\mathrm{b}}\left(x_{v}\right)\right)_{v \in t}\right)$. Clearly, $\Phi_{\mathrm{b}}(\Theta)$ is a $\bar{W}_{\mathrm{b}}$-labelled pointed tree and we easily check that the map is measurable. Moreover, if $\boldsymbol{\Theta}=\left(t, \varrho ;\left(X_{v}\right)_{v \in t}\right)$ has law $Q_{\mathrm{t}}^{+}$(resp. $\left.Q_{\mathrm{o}, \mathrm{t}}\right)$ then $\Phi_{\mathrm{b}}(\boldsymbol{\Theta})$ has law $Q_{\mathrm{t}}^{+\mathrm{b}}$ (resp. $Q_{\Phi_{\mathrm{b}}(\mathrm{o}), \mathrm{t}}^{\mathrm{b}}$ ).

\subsection{Metric properties of the range of free branching random walks}

We gather basic facts about the range of a free branching random walk in terms of the heights of the spatial positions in $\mathbb{W}_{[0,1]}^{*}$. Let $\mathbf{t}=(t, u) \in \overline{\mathrm{T}}^{\bullet}$ such that $|t|_{-}=-\infty$. For all $w \in t$, we recall that $\llbracket v, w \rrbracket$ is the shortest path (with respect to the graph-distance) that joins vertex $v$ to vertex $w$. Moreover, we set $\llbracket v, w \llbracket=\llbracket v, w \rrbracket \backslash\{w\}, \rrbracket v, w \rrbracket=\llbracket v, w \rrbracket \backslash\{v\}$ and $\rrbracket v, w \llbracket=\llbracket v, v \rrbracket \backslash\{v, w\}$; we also denote by $\rrbracket-\infty, v \rrbracket$ the lineage of $v$ : namely, $\rrbracket-\infty, v \rrbracket=$ $\left\{v_{\mid l} ; l \leq|v|\right\}$.

We next decompose a free branching random walk by first describing the heights of the vertices as a $\mathbb{Z}$-valued branching random walk $\left(\mathbf{h}_{v}\right)_{v \in t}$ and then explaining how to embed $\left(\mathbf{h}_{v}\right)_{v \in t}$ randomly in $\mathbb{W}_{[0,1]}^{*}$. More specifically, for all $v \in t$, let $\mathbf{h}_{v} \in \mathbb{Z}$ be such that

$$
\forall v \in t, \quad\left|\mathbf{h}_{v}-\mathbf{h}_{\overleftarrow{v}}\right|=1 \quad \text { and } \quad \inf _{w \in \llbracket v, u \rrbracket} \mathbf{h}_{w} \underset{|v| \rightarrow-\infty}{\longrightarrow}-\infty .
$$

Let $\left(U_{v}\right)_{v \in t}$ be a family of independent r.v. that are uniformly distributed on $[0,1]$. With $v \in t$, we associate a spatial position in $\mathbb{W}_{[0,1]}^{*}$ as follows. Since $\mathbf{h}$ takes arbitrary negative values on the lineage of $v$, for all integers $k \leq \mathbf{h}_{v}$,

$$
\text { there is a unique } v(k) \in \rrbracket-\infty, v \rrbracket \text { such that } \mathbf{h}_{v(k)}=\min _{w \in \llbracket(k), v \rrbracket} \mathbf{h}_{w}=k>\mathbf{h}_{\overleftarrow{v(k)}}
$$

Namely, $\left\{\overleftarrow{v(k)} ; k \leq \mathbf{h}_{v}\right\}$ is the set of vertices in the lineage of $v$ where $\mathbf{h}$ reaches a new infimum. In particular, note that $v=v\left(\mathbf{h}_{v}\right)$. Then, we set

$$
\forall v \in t, \quad X_{v}=\left(U_{v(k)}\right)_{k \leq \mathbf{h}_{v}} .
$$

By construction $\left|X_{v}\right|=\mathbf{h}_{v}, v \in t$. Then, $\left(t, u ;\left(X_{v}\right)_{v \in t}\right)$ is a $\mathbb{W}_{[0,1]}^{*}$-valued branching random walk satisfying

$$
X_{v}=\left\{\begin{array}{cl}
X_{\overleftarrow{v}} *\left(U_{v}\right) & \text { if }\left|X_{v}\right|-\left|X_{\overleftarrow{v}}\right|=1 \\
\overleftarrow{X_{\overleftarrow{v}}} & \text { if }\left|X_{v}\right|-\left|X_{\overleftarrow{v}}\right|=-1
\end{array}\right.
$$

We view $\left(X_{v}\right)_{v \in t}$ as a version of free branching random walk conditionally given the relative heights $\left(\mathbf{h}_{v}\right)_{v \in t}$ of the spatial positions. The following proposition is a key point to analyse the metric of the range of free branching random walks.

Proposition 3.5. Let $\mathbf{t}=(t, u) \in \overline{\mathbb{T}}^{\bullet},\left(\mathbf{h}_{v}, U_{v}, X_{v}\right)_{v \in t}$ be as above. Then, $\left\{X_{v} ; v \in t\right\}$ is a subtree of $\mathrm{W}_{[0,1]}^{*}$ as in Definition 2.3 and if we denote by $d_{\mathrm{gr}}$ it graph-distance, we get

$$
\text { P-a.s. for all } v, w \in t, \quad d_{\mathrm{gr}}\left(X_{v}, X_{w}\right)=\left|X_{v}\right|+\left|X_{w}\right|-2 \min _{v^{\prime} \in \llbracket v, w \rrbracket}\left|X_{v^{\prime}}\right| .
$$

Proof. We fix $v, w \in t$. By (2.6) we known that $d_{\mathrm{gr}}\left(X_{v}, X_{w}\right)=\left|X_{v}\right|+\left|X_{w}\right|-2\left|X_{v} \wedge X_{w}\right|$. Since $t$ is countable, to prove (3.7) it is enough to show that a.s. $\left|X_{v} \wedge X_{w}\right|=\min _{v^{\prime} \in \llbracket v, w \rrbracket}\left|\mathbf{h}_{v^{\prime}}\right|$. We prove it in two steps.

Step 1. Recall from (3.5) the definition of the $v(k), k \leq \mathbf{h}_{v}$ and define similarly the $w(k), k \leq \mathbf{h}_{w}$. We then set

$$
k=\max \left\{\ell \leq \mathbf{h}_{v}: v(\ell) \in \rrbracket-\infty, v \wedge w \rrbracket\right\} \quad \text { and } \quad m=\max \left\{\ell \leq \mathbf{h}_{w}: w(\ell) \in \rrbracket-\infty, v \wedge w \rrbracket\right\} .
$$


Let us prove that $k=\min _{\llbracket v, v \wedge w \rrbracket} \mathbf{h}$. If $k<\mathbf{h}_{v}$, then $v(k+1) \in \rrbracket v \wedge w, v \rrbracket$ and $\overleftarrow{v}(k+1) \in$ $\llbracket v \wedge w, v \rrbracket$; then $\mathbf{h}_{\overleftarrow{v}(k+1)}=\mathbf{h}_{v(k+1)}-1=k=\min _{\llbracket v, v \wedge w \rrbracket} \mathbf{h}$. If $k=\mathbf{h}_{v}$, then, by (3.5), $\mathbf{h}_{v}=\mathbf{h}_{v(k)}=\min _{\llbracket v, v(k) \rrbracket} \mathbf{h}$ and since $v(k) \in \rrbracket-\infty, v \wedge w \rrbracket$, we also get $k=\min _{\llbracket v, v \wedge w \rrbracket} \mathbf{h}$. Similarly, we get $m=\min _{\llbracket w, v \wedge w \rrbracket} \mathbf{h}$. By definition (3.6),

$$
X_{v}=X_{v(k)} *\left(U_{v(k+1)}, \ldots, U_{v\left(\mathbf{h}_{v}\right)}\right) \quad \text { and } \quad X_{w}=X_{w(m)} *\left(U_{w(m+1)}, \ldots, U_{w\left(\mathbf{h}_{w}\right)}\right)
$$

with the observation that $X_{v}=X_{v(k)}$ (resp. $X_{w}=X_{w(m)}$ ) if $k=\mathbf{h}_{v}$ (resp. if $m=\mathbf{h}_{w}$ ). Without loss of generality, we can assume that $k \leq m$. Then, $v(k-i)=w(k-i)$, for all $i \in \mathbb{N}$ and

$$
X_{w(m)}=X_{v(k)} *\left(U_{w(k+1)}, \ldots, U_{w(m)}\right) .
$$

Step 2. We conclude the proof by proving that

$$
X_{v} \wedge X_{w}=X_{v(k)} .
$$

Suppose first that $k<m$ and that $k<\mathbf{h}_{v}$. Then $w(k+1) \in \rrbracket-\infty, v \wedge w \rrbracket$ but $v(k+1) \in \rrbracket v \wedge w, v \rrbracket$. Thus, $w(k+1) \neq v(k+1)$ and since the $\left(U_{v^{\prime}}\right)_{v^{\prime} \in t}$ are independent with a diffuse law, a.s. $U_{w(k+1)} \neq U_{v(k+1)}$. By (3.8) and (3.9), we get (3.10). If $k<m$ and $k=\mathbf{h}_{v}$, then $X_{v}=X_{v(k)}$ and we immediately get (3.10) by (3.8) and (3.9).

Next, suppose that $k=m$. Thus $X_{w(m)}=X_{v(k)}$ by (3.9); if $k=m<\mathbf{h}_{v} \wedge \mathbf{h}_{w}$, then $v(k+1) \in \rrbracket v \wedge w, v \llbracket$ and $w(k+1)=w(m+1) \in \rrbracket v \wedge w, w \rrbracket$; therefore $w(k+1) \neq v(k+1)$, which implies that a.s. $U_{w(k+1)} \neq U_{v(k+1)}$ and (3.10) consequently. If $k=m$ and $k=\mathbf{h}_{v}$ (resp. $m=\mathbf{h}_{w}$ ), then $X_{v}=X_{v(k)}=X_{w(m)}\left(\right.$ resp. $\left.X_{w}=X_{w(m)}=X_{v(k)}\right)$ and (3.8) and (3.9) also entail (3.10). This completes the proof of (3.10).

We shall use Proposition 3.5 under the following form.

Corollary 3.6. Let $t^{\prime} \in \mathbb{T}$ be a finite rooted ordered tree. Let $\left(t^{\prime}, \varnothing ;\left(Y_{v}\right)_{v \in t^{\prime}}\right)$ be a $\mathbb{W}_{[0,1]^{-}}$ valued branching random walk with law $Q_{\mathrm{t}^{\prime}}^{+}$as in Definition $3.1(i i)$. Then, a.s. for all $v, w \in t^{\prime}$, we get $d_{\mathrm{gr}}\left(Y_{v}, Y_{w}\right)=\left|Y_{v}\right|+\left|Y_{w}\right|-2 \min _{v^{\prime} \in \llbracket v, w \rrbracket}\left|Y_{v^{\prime}}\right|$.

Proof. For all $k \in \mathbb{Z}_{-}$we set $\varrho_{k}=\left(a_{n}\right)_{n \leq k}$, where $a_{n}=1$ for all $n \in \mathbb{Z}_{-}$, and we also define $t=\left\{\varrho_{0} * u ; u \in t^{\prime}\right\} \cup\left\{\varrho_{k} ; k \in \mathbb{Z}_{-}\right\}$. Then, $t \in \overline{\mathbb{T}}^{\bullet},|t|_{-}=-\infty$ and $t^{\prime}=\theta_{\varrho_{0}} t$. Let $\left(\mathbf{h}_{v}^{\prime}\right)_{v \in t^{\prime}}$ be distributed as an $\mathbb{N}$-valued branching random walk whose initial position $\mathbf{h}_{\varnothing}^{\prime}$ is 0 and whose transition kernel $q\left(y, d y^{\prime}\right)$ is that of the simple symmetric random walk on $\mathbb{N}$ reflected at 0 : namely, $q\left(0, d y^{\prime}\right)=\delta_{1}$ and $q\left(y, d y^{\prime}\right)=\frac{1}{2}\left(\delta_{y-1}\left(d y^{\prime}\right)+\delta_{y+1}\left(d y^{\prime}\right)\right)$, for all integers $y^{\prime} \geq 1$ (see (3.1)). We next set $\mathbf{h}_{\varrho_{0} * v}=\mathbf{h}_{v}^{\prime}$, for all $v \in t^{\prime}$ and $\mathbf{h}_{\varrho_{k}}=k$, for all $k \in \mathbb{Z}_{-}$. Clearly $\mathbf{h}$ satisfies (3.4). We assume that $\left(U_{v}\right)_{v \in t}$ is independent from $\left(\mathbf{h}_{v}\right)_{v \in t}$ and we define $\left(X_{v}\right)_{v \in t}$ as in (3.6). For all $v \in t^{\prime}$, we finally set $Y_{v}=\theta_{X_{\varrho_{0}}} X_{\varrho_{0} * v}$. Then, it is easy to see that $\left(t^{\prime}, \varnothing,\left(Y_{v}\right)_{v \in t^{\prime}}\right)$ has law $Q_{\mathbf{t}^{\prime}}^{+}$. Since $\mathbf{h}_{\varrho_{0} * v} \geq 0$, for all $v \in t^{\prime}$, we get $d_{\mathrm{gr}}\left(X_{\varrho_{0} * v}, X_{\varrho_{0} * w}\right)=d_{\mathrm{gr}}\left(Y_{v}, Y_{w}\right)$ and $\left|X_{\varrho_{0} * v}\right|=\left|Y_{v}\right|$, for all $v, w \in t^{\prime}$, which implies the desired result by (3.7).

We next consider subranges of free branching random walks. More precisely, let $\mathbf{t}=(t, u) \in \overline{\mathbb{T}}^{\bullet}$ be such that $|t|_{-}=-\infty$; for all $v \in t$, let $\mathbf{h}_{v} \in \mathbb{Z}$ satisfy (3.4); let $\left(U_{v}\right)_{v \in t}$ be a family of independent r.v. that are uniformly distributed on $[0,1]$ and let $\left(X_{v}\right)_{v \in t}$ be derived from $\left(U_{v}\right)_{v \in t}$ as specified in (3.5) and (3.6). Let $\mathbf{a} \subset t$ and $v_{0} \in \mathbf{a}$ be such that

$$
\forall v \in \mathbf{a} \backslash\left\{v_{0}\right\}, \overleftarrow{v} \in \mathbf{a}, v \text { is a descendent of } v_{0} \text { (namely } v=v_{0} *\left(\theta_{v_{0}} v\right) \text { ) and } \mathbf{h}_{v} \geq \mathbf{h}_{v_{0}}
$$

Observe that for all $v \in \mathbf{a}, X_{v_{0}}$ is a prefix of $X_{v}$ and it makes sense to define "the subrange":

$$
\mathcal{R}(\mathbf{a})=\left\{\theta_{X_{v_{0}}} X_{v} ; v \in \mathbf{a}\right\}
$$


that is a subtree of $\mathbb{W}_{[0,1]}^{*}$ whose elements have a null depth. We define the following.

$$
\forall v, w \in t, \quad d(v, w)=\mathbf{h}_{v}+\mathbf{h}_{w}-2 \min _{\llbracket v, w \rrbracket} \mathbf{h} .
$$

Note that $\llbracket v, w \rrbracket \subset \mathbf{a}$; thus, the pseudo-metric $d$ on $\mathbf{a} \times \mathbf{a}$ only depends on $\mathbf{a}$ and on $\left(\mathbf{h}_{v}-\mathbf{h}_{v_{0}}\right)_{v \in \mathbf{a}}$. We define the relation $\sim$ on a by setting $v \sim w$ if and only if $d(v, w)=0$ and we introduce

$$
T(\mathbf{a})=\mathbf{a} / \sim, \quad \operatorname{proj}: \mathbf{a} \rightarrow T(\mathbf{a}), \text { the canonical projection, } \quad r=\operatorname{proj}\left(v_{0}\right)
$$

and we keep denoting $d$ the (true) metric induced by $d$ on $T(\mathbf{a})$. If $\mathbf{t}=(t, \varrho) \in \overline{\mathbb{T}}^{\bullet}$ and $\left(\mathbf{h}_{v}, U_{v}, X_{v}\right)_{v \in t}$ are as in Proposition 3.5, then (3.7) implies that $(T(\mathbf{a}), d)$ is a graph-tree that is isometric to the subtree $\mathcal{R}(\mathbf{a})$. More precisely, let $x \in T(\mathbf{a})$ and let $v, w \in \mathbf{a}$ such that $\operatorname{proj}(v)=\operatorname{proj}(w)=x$; by (3.7), we get a.s. $X_{v}=X_{w}$ and it makes sense to set $Z_{x}=\theta_{X_{v_{0}}} X_{v}$. Then (3.7) asserts that $Z: T(\mathbf{a}) \rightarrow \mathcal{R}(\mathbf{a})$ is an isometry:

$$
\forall x, y \in T(\mathbf{a}), \quad d(x, y)=\left|Z_{x}\right|+\left|Z_{y}\right|-2\left|Z_{x} \wedge Z_{y}\right|=d_{\mathrm{gr}}\left(Z_{x}, Z_{y}\right) .
$$

Thus, the graph-metric of the subtree $\mathcal{R}(\mathbf{a})$ only depends on a and on $\left(\mathbf{h}_{v}-\mathbf{h}_{v_{0}}\right)_{v \in \mathbf{a}}$. Next, the conditional law of $\mathcal{R}(\mathbf{a})$ given $T(\mathbf{a})$ is characterized as follows. For all $x \in T(\mathbf{a}) \backslash\{r\}$, let $V_{x}$ be the unique real number of $[0,1]$ such that

$$
Z_{x}=\overleftarrow{Z}_{x} *\left(V_{x}\right)
$$

We easily check the following.

Conditionally given $T(\mathbf{a})$, the $V_{x}$ are i.i.d. [0,1]-uniform r.v.

Recall from Definition 3.3 the b-contraction map $\Phi_{\mathrm{b}}: \overline{\mathrm{W}}_{[0,1]} \rightarrow \overline{\mathrm{W}}_{\mathrm{b}}$. Then, first note that $\left|Z_{x} \wedge Z_{y}\right| \leq\left|\Phi_{\mathrm{b}}\left(Z_{x}\right) \wedge \Phi_{\mathrm{b}}\left(Z_{y}\right)\right|$, where common ancestors are taken respectively in $\mathbb{W}_{[0,1]}$ and in $\mathbb{W}_{\mathrm{b}}$. Moreover, (3.16) implies that $\mathbf{P}\left(\left|\Phi_{\mathrm{b}}\left(Z_{x}\right) \wedge \Phi_{\mathrm{b}}\left(Z_{y}\right)\right|-\left|Z_{x} \wedge Z_{y}\right| \geq k\right) \leq \mathrm{b}^{-k}$, for all $k \in \mathbb{N}$. This inequality combined with the argument of the proof of Corollary 3.6 implies the following lemma that will be used in Theorem 1.2.

Lemma 3.7. Let $t \in \mathbb{T}$ be a finite rooted ordered tree. Let $\left(t, \varnothing ;\left(Y_{v}\right)_{v \in t}\right)$ be a $\mathbb{W}_{[0,1]}$-valued branching random walk with law $Q_{\mathrm{t}}^{+}$as in Definition $3.1(i i)$. Recall the b-contraction map $\Phi_{\mathrm{b}}: \mathbb{W}_{[0,1]} \rightarrow \mathbb{W}_{d}$ from Definition 3.3 and recall that $\left(t, \varnothing,\left(\Phi_{\mathrm{b}}\left(Y_{v}\right)\right)_{v \in t}\right)$ has law $Q_{\mathrm{t}}^{+\mathrm{b}}$ as in Definition $3.1(i)$. We denote the graph distance on $\mathbb{W}_{[0,1]}$ and $\mathbb{W}_{\mathrm{b}}$ in the same way by $d_{\mathrm{gr}}$. Then, for all $v, w \in t$, there exists an $\mathbb{N}$-valued r.v. $G_{v, w}$ such that

$$
2 G_{v, w}=d_{\mathrm{gr}}\left(X_{v}, X_{w}\right)-d_{\mathrm{gr}}\left(\Phi_{\mathrm{b}}\left(X_{v}\right), \Phi_{\mathrm{b}}\left(X_{w}\right)\right) \quad \text { and } \quad \mathbf{P}\left(G_{v, w} \geq k\right) \leq \mathrm{b}^{-k}, k \in \mathbb{N} .
$$

\subsection{A coupling between $W_{b}$ - and $W_{b}^{*}$-valued branching random walks}

This section is devoted to the proof of the following proposition.

Proposition 3.8. Let $t \in \mathbb{T}$ be a finite rooted ordered tree as in Definition 2.1 (namely, $\left.|t|_{-}=0\right)$. We set $\mathbf{t}=(t, \varnothing)$. For all $n \in\{0, \ldots, \# t-1\}$, we set $t_{n}=\left\{v \in t: v \leq_{t} v_{n}\right\}$ where $v_{n}$ is the $n$-th smallest vertex of $t$ with respect to the lexicographical order $\leq_{t}$. Then, there exists two branching random walks $\boldsymbol{\Theta}_{\mathrm{b}}=\left(t, \varnothing ;\left(Y_{v}\right)_{v \in t}\right)$ and $\boldsymbol{\Theta}_{\mathrm{b}}^{+}=\left(t, \varnothing ;\left(Y_{v}^{+}\right)_{v \in t}\right)$ that satisfy the following.

(a) $\Theta_{\mathrm{b}}^{+}$is a $\mathbb{W}_{\mathrm{b}}$-valued branching random walk with law $Q_{\mathrm{t}}^{+\mathrm{b}}$ as in Definition $3.1(i)$.

(b) $\mathbf{o}_{\mathrm{b}}:=Y_{\varnothing}$ is a $\mathbb{Z}_{-}$-indexed sequence of independent r.v. that are uniformly distributed on $\{1, \ldots, \mathrm{b}\}$ and conditionally given $\mathbf{o}_{\mathrm{b}}, \boldsymbol{\Theta}_{\mathrm{b}}$ is a $\overline{\mathrm{W}}_{\mathrm{b}}$-valued branching random walk with law $Q_{\mathbf{o}_{b}, \mathrm{t}}^{\mathrm{b}}$ as in Definition 3.1 (iii).

(c) For all $c \in \mathbb{N}^{*}$, there exists an event $B_{c}$ such that $\mathbf{P}\left(B_{c}\right) \leq 2 \mathrm{~b}^{-c}(\# t)^{3}$ and such that on $\Omega \backslash B_{c}$,

$$
\forall n \in\{0, \ldots, \# t-1\},\left|\#\left\{Y_{v} ; v \in t_{n}\right\}-\#\left\{Y_{v}^{+} ; v \in t_{n}\right\}\right| \leq \#\left\{v \in t:\left|Y_{v}^{+}\right| \leq c+1\right\} .
$$


Overlap of independent trees. We first prove a result concerning the overlap of independent trees that are randomly embedded in the b-ary tree $\mathbb{W}_{\mathrm{b}}$. More precisely, let $\left(\mathrm{T}_{u}, r(u)\right), u \in S$ be a finite family of rooted graph-trees (not necessarily ordered) equipped with their graph-distance $d_{\mathrm{gr}}$. To simplify notation, we set $|x|=d_{\mathrm{gr}}(r(u), x)$, for all $x \in \mathrm{T}_{u}$. Let $x, y \in \mathrm{T}_{u}$; recall that $\llbracket x, y \rrbracket$ stands for the shortest path joining $x$ to $y$ and that $x \wedge y$ is the most recent common ancestor of $x$ and $y$ in $\mathrm{T}_{u}$ rooted at $r(u)$. Namely, $\llbracket r(u), x \rrbracket \cap \llbracket r(u), y \rrbracket=\llbracket r(u), x \wedge y \rrbracket$. Let $x \in \mathrm{T}_{u} \backslash\{r(u)\}$; for all $i \in\{1, \ldots,|x|\}$ we denote by $x_{i}$ the unique ancestor of $x$ at height $i$. Let $V_{x}^{u}, x \in \mathrm{T}_{u}, u \in S$ be independent uniform r.v. on $\{1, \ldots, b\}$. Then, we define the random word:

$$
Z_{x}^{u}=\left(V_{x_{1}}^{u}, V_{x_{2}}^{u}, \ldots, V_{x_{|x|-1}}^{u}, V_{x}^{u}\right)
$$

We also set $Z_{r(u)}^{u}=\varnothing$ and we introduce the following random subsets of the b-ary tree $\mathrm{W}_{\mathrm{b}}$ :

$$
\forall u \in S, \quad \mathrm{R}_{u}:=\left\{Z_{x}^{u} ; x \in \mathrm{T}_{u}\right\} .
$$

For all $c \in \mathbb{N}^{*}$, we also set $M_{c}=\sum_{u \in S} \#\left\{x \in \mathrm{T}_{u}:|x| \leq c\right\}$.

Lemma 3.9. We keep the notation from above. Let $c \in \mathbb{N}^{*}$. Let $w_{u} \in \mathbb{W}_{\mathrm{b}}, u \in S$. Then, there exists an event $A_{c}$ of probability $\mathbf{P}\left(A_{c}\right) \leq \mathrm{b}^{-c} M_{c}^{2}$ such that on $\Omega \backslash A_{c}$,

$$
0 \leq \sum_{u \in S} \# \mathrm{R}_{u}-\#\left(\bigcup_{u \in S} w_{u} *\left(\mathrm{R}_{u}\right)\right) \leq M_{c} .
$$

Proof. Let $u$ and $u^{\prime}$ be distinct elements of $S$; let $x \in \mathrm{T}_{u}$ and $y \in \mathrm{T}_{u^{\prime}}$ be such that $|x|=|y|=c$. We introduce the event $A(x, y)=\left\{\left(w_{u} * Z_{x}^{u}\right) \wedge\left(w_{u^{\prime}} * Z_{y}^{u^{\prime}}\right) \in\left\{w_{u} * Z_{x}^{u}, w_{u^{\prime}} * Z_{y}^{u^{\prime}}\right\}\right\}$. Note that on $A(x, y), w_{u} \wedge w_{u^{\prime}} \in\left\{w_{u}, w_{u^{\prime}}\right\}$; so without loss of generality, we can suppose that $\left|w_{u}\right| \leq\left|w_{u^{\prime}}\right|$ and we easily check that $\mathbf{P}\left(A(x, y) \mid Z_{y}^{u^{\prime}}\right)=\mathrm{b}^{-c}$ and thus $\mathbf{P}(A(x, y))=\mathrm{b}^{-c}$. We then set for all $u \in S$,

$$
\mathrm{T}_{u}(c)=\left\{x \in \mathrm{T}_{u}:|x|=c\right\} \quad \text { and } \quad A_{c}=\bigcup\left\{A(x, y) ; x \in \mathrm{T}_{u}(c), y \in \mathrm{T}_{u^{\prime}}(c), u, u^{\prime} \in S \text {, distinct }\right\} .
$$

Thus, $\mathbf{P}\left(A_{c}\right) \leq \mathrm{b}^{-c} \sum_{u, u^{\prime} \in S, u \neq u^{\prime}} \# \mathrm{~T}_{u}(c) \# \mathrm{~T}_{u^{\prime}}(c) \leq \frac{1}{2} \mathrm{~b}^{-c}\left(\sum_{u \in S} \# \mathrm{~T}_{u}(c)\right)^{2} \leq \mathrm{b}^{-c} M_{c}^{2}$.

The first inequality in (3.19) is true everywhere on $\Omega$. For the second inequality in (3.19), we argue deterministically on $\Omega \backslash A_{c}$. Let $u \in S$. We first set $R_{u}(c)=\left\{w_{u} * Z_{x}^{u} ; x \in\right.$ $\left.\mathrm{T}_{u}(c)\right\}$. Let $u^{\prime} \in S$ be distinct from $u$; suppose that $W \in R_{u}(c)$ and that $W^{\prime} \in R_{u^{\prime}}(c)$; by definition of $A_{c}, W \wedge W^{\prime} \notin\left\{W, W^{\prime}\right\}$ and $\theta_{W}\left(w_{u} * \mathrm{R}_{u}\right) \cap \theta_{W^{\prime}}\left(w_{u^{\prime}} * \mathrm{R}_{u^{\prime}}\right)=\emptyset$. Moreover, if $W, W^{\prime} \in R_{u}(c)$ are distinct, since $|W|=\left|W^{\prime}\right|=c+\left|w_{u}\right|$, we also get $W \wedge W^{\prime} \notin\left\{W, W^{\prime}\right\}$ and thus $\theta_{W}\left(w_{u} * \mathrm{R}_{u}\right) \cap \theta_{W^{\prime}}\left(w_{u} * \mathrm{R}_{u}\right)=\emptyset$. Consequently, on $\Omega \backslash A_{c}$, the subsets $\theta_{W}\left(w_{u} * \mathrm{R}_{u}\right)$, $W \in R_{u}(c), u \in S$, are pairwise disjoint.

Now observe that $\left(w_{u} * \mathrm{R}_{u}\right) \backslash\left(\bigcup_{W \in R_{u}(c)} \theta_{W}\left(w_{u} * \mathrm{R}_{u}\right)\right)=\left\{w_{u} * Z_{x}^{u} ;|x|<c\right\}$ and note that $\#\left(\left\{w_{u} * Z_{x}^{u} ;|x|<c\right\}\right) \leq \#\left(\left\{x \in \mathrm{T}_{u}:|x|<c\right\}\right)$. Thus, on $\Omega \backslash A_{c}$, we get

$$
\begin{aligned}
\left(\sum_{u \in S} \# \mathrm{R}_{u}\right)-M_{c} & =\sum_{u \in S}\left(\# \mathrm{R}_{u}-\#\left\{x \in \mathrm{T}_{u}:|x|<c\right\}\right) \\
& \leq \sum_{u \in S}\left(\#\left(w_{u} * \mathrm{R}_{u}\right)-\#\left(\left\{w_{u} * Z_{x}^{u} ;|x|<c\right\}\right)\right) \\
& \leq \sum_{u \in S} \#\left(\bigcup_{W \in R_{u}(c)} \theta_{W}\left(w_{u} * \mathrm{R}_{u}\right)\right) \\
& =\#\left(\bigcup_{u \in S} \bigcup_{W \in R_{u}(c)} \theta_{W}\left(w_{u} *\left(\mathrm{R}_{u}\right)\right)\right) \leq \#\left(\bigcup_{u \in S} w_{u} *\left(\mathrm{R}_{u}\right)\right),
\end{aligned}
$$

that implies the desired result. 
Path coupling. We first state the following elementary coupling.

Lemma 3.10. Let $K: \mathbb{N} \rightarrow \mathbb{N}$ be such that $K(0)=0$ and $K(2 p+1)=K(2 p+2)=2 p+2$, for all $p \in \mathbb{N}$. Let $\left(\mathbf{h}_{n}\right)_{n \in \mathbb{N}}$ be a $\mathbb{Z}$-valued simple symmetric random walk such that a.s. $\mathbf{h}_{0}=0$. For all $n \in \mathbb{N}$, we set $I_{n}=\inf _{0 \leq k \leq n} \mathbf{h}_{k}$ and $\mathbf{h}_{n}^{+}=\mathbf{h}_{n}+K\left(-I_{n}\right)$. Then, $\mathbf{h}^{+}$is an $\mathbb{N}$-valued simple symmetric random walk reflected at 0 .

Proof. For all $n \geq 1$, set $\xi_{n}=\mathbf{h}_{n}-\mathbf{h}_{n-1}$; the r.v. are i.i.d. and uniform on $\{1,-1\}$. Observe that $\mathbf{h}_{n}^{+}=\mathbf{h}_{n}-I_{n}+\mathbb{1}_{\left\{I_{n} \text { odd }\right\}}$. It is easy to check that $\mathbf{h}_{n+1}^{+}-\mathbf{h}_{n}^{+}=\xi_{n+1} \mathbb{1}_{\left\{\mathbf{h}_{n}^{+} \geq 1\right\}}+\mathbb{1}_{\left\{\mathbf{h}_{n}^{+}=0\right\}}$, which implies the desired result.

The next lemma state the branching random walk version of this coupling.

Lemma 3.11. Let $t \in \mathbb{T}$ be a rooted ordered tree as in Definition 2.1 (namely, $|t|_{-}=0$ ). Let $\left(t, \varnothing ;\left(\mathbf{h}_{u}\right)_{u \in t}\right)$ be a $\mathbb{Z}$-valued branching random walk whose spatial motion is that of a simple symmetric random walk on $\mathbb{Z}$ and whose initial position is $\mathbf{h}_{\varnothing}=0$. Recall the function $K$ from Lemma 3.10 and set

$$
\forall u \in t, \quad I_{u}=\min _{u^{\prime} \in \llbracket \varnothing, u \rrbracket} \mathbf{h}_{u^{\prime}} \quad \text { and } \quad \mathbf{h}_{u}^{+}=\mathbf{h}_{u}+K\left(-I_{u}\right) .
$$

Then, $\left(t, \varnothing ;\left(\mathbf{h}_{u}^{+}\right)_{u \in t}\right)$ is a branching random walk whose spatial motion is that of an $\mathbb{N}$-valued simple symmetric random walk reflected at 0 and whose initial position is $\mathbf{h}_{\varnothing}^{+}=0$.

Proof. We denote by $q_{+}(x, d y)$ the transition kernel of the $\mathbb{N}$-valued simple symmetric random walk reflected at 0 : namely, $q_{+}(0, d y)=\delta_{1}(d y)$ and $q_{+}(x, d y)=\frac{1}{2}\left(\delta_{x-1}(d y)+\right.$ $\left.\delta_{x+1}(d y)\right)$, if $x \geq 1$. For all $n \in \mathbb{N}$, we set $t_{\mid n}=\{u \in t:|u| \leq n\}$ and we assume the following property

$\left(P_{n}\right)$ : the spatial motion of the branching random walk $\mathbf{\Theta}_{n}=\left(t_{\mid n}, \varnothing ;\left(\mathbf{h}_{u}^{+}\right)_{u \in t_{\mid n}}\right)$ is that of a $\mathbb{N}$-valued simple symmetric random walk reflected at 0 with initial position $\mathbf{h}_{\varnothing}^{+}=0$.

We next set $S=\{u \in t:|u|=n+1\}$ and $\xi_{u}=\mathbf{h}_{u}-\mathbf{h}_{\overleftarrow{u}}$ for all $u \in S$; the r.v. $\left(\xi_{u}\right)_{u \in S}$ are independent from $\boldsymbol{\Theta}_{n}$ and they are also i.i.d. and uniform on $\{1,-1\}$; by definition of $\mathbf{h}^{+}$, we also get $\mathbf{h}_{u}^{+}=\mathbf{h}_{\frac{t}{u}}^{+}+\xi_{u} \mathbb{1}_{\left\{\mathbf{h}_{\frac{\pi}{u}}^{+} \geq 1\right\}}+\mathbb{1}_{\left\{\mathbf{h}_{\frac{1}{u}}^{+}=0\right\}}$. This entails that the r.v. $\mathbf{h}_{u}^{+}, u \in S$ are conditionally independent given $\boldsymbol{\Theta}_{n}$ and that the conditional law of $\mathbf{h}_{u}^{+}$is $q_{+}\left(\mathbf{h}_{\overleftarrow{u}}^{+}, d y\right)$. This shows that $\left(P_{n}\right)$ implies $\left(P_{n+1}\right)$, which recursively proves the lemma since $\left(P_{0}\right)$ holds true trivially.

The coupling of $\Theta_{\mathrm{b}}$ and $\Theta_{\mathrm{b}}^{+}$. Let $t \in \mathbb{T}$ be a finite rooted ordered tree as in Definition 2.1 (namely, $|t|_{-}=0$ ). We shall use the notation $\mathbf{t}=(t, \varnothing)$ for the pointed tree. We denote by $\varrho=(\ldots, 1, \ldots, 1)$ the infinite sequence of 1 indexed by $\mathbb{Z}_{-}$. For all $l \in \mathbb{Z}_{-}, \varrho_{\mid l}$ is the infinite sequence of 1 indexed by the integers $\leq l$. We then set

$$
t^{*}=\left\{\varrho_{\mid l} ; l \in \mathbb{Z}_{-}\right\} \cup\{\varrho * u ; u \in t\} .
$$

Then we define $\mathbf{t}^{*}:=\left(t^{*}, \varrho\right) \in \overline{\mathbb{T}}^{\bullet}$ and we observe that $\left|t^{*}\right|_{-}=-\infty$.

Let $\left(t^{*}, \varrho ;\left(\mathbf{h}_{v}^{*}\right)_{v \in t^{*}}\right)$ be a $\mathbb{Z}$-valued branching random walk whose transition kernel is that of a simple symmetric random walk on $\mathbb{Z}$ and whose "initial" position is $\mathbf{h}_{e}^{*}=0$. Let $\left(U_{v}^{*}\right)_{v \in t^{*}}$ be independent r.v. that are uniformly distributed on $[0,1]$ and that are independent from $\left(\mathbf{h}_{v}^{*}\right)_{v \in t^{*}}$. We define $\left(X_{v}^{*}\right)_{v \in t^{*}}$ as in (3.5) and (3.6): namely, $X_{v}^{*}=$ $\left(U_{v(k)}^{*}\right)_{k \leq \mathbf{h}_{v}^{*}}$, where $v(k) \in \rrbracket-\infty, v \rrbracket, \mathbf{h}_{v(k)}^{*}=k$ and where $\left\{\overleftarrow{v}(k) ; k \leq \mathbf{h}_{v}^{*}\right\}$ is the set of vertices of $\rrbracket-\infty, v \rrbracket$ where $\mathbf{h}^{*}$ reaches a new strict infimum. We set $\mathbf{o}=X_{e}^{*}$ that is a $\mathbb{Z}_{-}$-indexed sequence of mutually independent r.v. that are uniformly distributed on 
$[0,1]$. We easily see that conditionally given $\mathbf{o}, \boldsymbol{\Theta}^{*}=\left(t^{*}, \varrho ;\left(X_{v}^{*}\right)_{v \in t^{*}}\right)$ has law $Q_{\mathbf{o}, \mathbf{t}^{*}}$ as in Definition $3.1(\mathrm{iv})$. Then we set the following

$$
\forall u \in t, \quad U_{u}=U_{\varrho * u}^{*}, X_{u}=X_{\varrho * u}^{*}, \mathbf{h}_{u}=\mathbf{h}_{\varrho * u}^{*}, Y_{u}=\Phi_{\mathrm{b}}\left(X_{u}\right),
$$

and we also set $\boldsymbol{\Theta}=\left(t, \varnothing ;\left(X_{u}\right)_{u \in t}\right)$ and $\boldsymbol{\Theta}_{\mathrm{b}}=\left(t, \varnothing ;\left(Y_{u}\right)_{u \in t}\right)$, where we recall the definition of the b-contraction map $\Phi_{\mathrm{b}}$ from Remark 3.3. Clearly, conditionally given $\mathbf{o}, \boldsymbol{\Theta}$ has law $Q_{\mathbf{o}, \mathbf{t}}$ as in Definition $3.1(i v)$ and by Remark 3.3, the conditional law of $\Theta_{\mathrm{b}}$ is $Q_{\Phi_{\mathrm{b}}(\mathrm{o}), \mathrm{t}}^{\mathrm{b}}$ as in Definition 3.1 (iii). Namely, $\boldsymbol{\Theta}_{\mathrm{b}}$ is distributed as in Proposition $3.8(b)$.

Let $\left(U_{u}^{\prime}\right)_{u \in t}$ be i.i.d. r.v. that are uniformly distributed on $[0,1]$. We suppose that $\left(U_{u}^{\prime}\right)_{u \in t}$ is independent from $\left(U_{v}^{*}\right)_{v \in t^{*}}$ (and thus from $\left(U_{u}\right)_{u \in t}$ ) and from $\left(\mathbf{h}_{v}^{*}\right)_{v \in t^{*}}$. We next construct $\boldsymbol{\Theta}_{\mathrm{b}}^{+}$thanks to $\left(U_{u}^{\prime}, U_{u}, \mathbf{h}_{u}\right)_{u \in t}$ as follows: For all $u \in t$, we define $I_{u}$ and $\mathbf{h}_{u}^{+}$by (3.20) in Lemma 3.11 and we introduce the following notation.

$$
\forall p \in \mathbb{N}, \quad S_{p}=\left\{u \in t:-p=I_{u}<I_{\overleftarrow{u}}\right\}, \quad S=\bigcup_{p \in \mathbb{N}} S_{p}, U_{u}^{+}= \begin{cases}U_{u}^{\prime} & \text { if } u \in \bigcup_{p \in \mathbb{N}} S_{2 p+1} \\ U_{u} & \text { otherwise. }\end{cases}
$$

The (disjoint) union $S$ is clearly a finite set, since $t$ is a finite tree. Then, $\left(U_{u}^{+}\right)_{u \in t}$ are i.i.d. $[0,1]$-uniform r.v. that are independent from $\left(\mathbf{h}_{u}^{+}\right)_{u \in t}$. Recall from Lemma 3.11 that $\left(t, \varnothing ;\left(\mathbf{h}_{u}^{+}\right)_{u \in t}\right)$ is a branching random walk whose spatial motion is that of an $\mathbb{N}$-valued simple symmetric random walk reflected at 0 and whose initial position is $\mathbf{h}_{\varnothing}^{+}=0$. In particular, $\mathbf{h}_{u}^{+} \geq 0$ for all $u \in t$. We then define $\left(X_{u}^{+}\right)_{u \in t}$ and $\left(Y_{u}^{+}\right)_{u \in t}$ as follows.

- If $\mathbf{h}_{u}^{+}=0$, then we set $X_{u}^{+}=Y_{u}^{+}=\varnothing$.

- If $\mathbf{h}_{u}^{+} \geq 1$, then for all $i \in\left\{1, \ldots, \mathbf{h}_{u}^{+}\right\}$, we denote by $u(i)$ is the unique $v \in \llbracket \varnothing, u \rrbracket$ such that $\mathbf{h}_{\overleftarrow{v}}^{+}<i=\mathbf{h}_{v}^{+}=\min _{\llbracket v, u \rrbracket} \mathbf{h}^{+}$and we set

$$
X_{u}^{+}=\left(U_{u(1)}^{+}, \ldots, U_{u\left(\mathbf{h}_{u}^{+}\right)}^{+}\right) \text {and } Y_{u}^{+}=\Phi_{\mathrm{b}}\left(X_{u}^{+}\right) .
$$

We set $\boldsymbol{\Theta}^{+}=\left(t, \varnothing ;\left(X_{u}^{+}\right)_{u \in t}\right)$ and $\boldsymbol{\Theta}_{\mathrm{b}}^{+}=\left(t, \varnothing ;\left(Y_{u}^{+}\right)_{u \in t}\right)$. Then, observe that $\mathbf{h}_{u}^{+}=\left|X_{u}^{+}\right|$and that $X_{u}^{+}=X_{\breve{u}}^{+} *\left(U_{u}^{+}\right)$if $\mathbf{h}_{u}^{+}=\mathbf{h}_{\overleftarrow{u}}^{+}+1$ and note that $X_{u}^{+}$is the parent of $X_{\breve{u}}^{+}$if $\mathbf{h}_{u}^{+}=\mathbf{h}_{\breve{u}}^{+}-1$. Thus, it proves that $\Theta^{+}$as law $Q_{\mathrm{t}}^{+}$as in Definition $3.1(\mathrm{ii})$ and by Remark 3.3, $\Theta_{\mathrm{b}}^{+}$is distributed as in Proposition $3.8(a)$ (namely its law is $Q_{\mathrm{t}}^{+\mathrm{b}}$ as in Definition $3.1(i)$ ).

Proof of Proposition 3.8. We keep the previous notations. We fix $n \in\{0, \ldots, \# t-1\}$. Recall that $t_{n}=\left\{v \in t: v \leq_{t} v_{n}\right\}$ where $v_{n}$ is the $n$-th smallest vertex of $t$ in the lexicographical order $\leq_{t}$ on $t$. Note that $t_{n} \in \mathbb{T}$, namely it is a rooted ordered tree with null depth. Recall from (3.21) the definition of $S$. For all $u \in S$, we set

$\mathbf{a}_{u}=\left\{v \in t_{n}: v:=u * \theta_{u} v\right.$ and $\left.\min _{w \in \llbracket u, v \rrbracket} \mathbf{h}_{w}=\mathbf{h}_{u}\right\} \subseteq t \quad$ and $\quad \mathcal{R}\left(\mathbf{a}_{u}\right)=\left\{\theta_{X_{u}} X_{v} ; v \in \mathbf{a}_{u}\right\} \subseteq \overline{\mathbb{W}}_{[0,1]}$.

Note that $\mathbf{a}_{u}$ depends on $n$ and that if $u \notin t_{n}$, then $\mathbf{a}_{u}$ and $\mathcal{R}\left(\mathbf{a}_{u}\right)$ are empty. If $u \in t_{n}$, then the previous definitions make sense because $\mathbf{a}_{u}$ satisfies (3.11) (with $u=v_{0}$ ) and $X_{u}$ is necessarily a prefix of $X_{v}$ since $v$ is a descendent of $u$ and $I_{v}=I_{u}$. Observe that $\mathcal{R}\left(\mathbf{a}_{u}\right)$ is a subtree of $\overline{\mathrm{W}}_{[0,1]}$ as in Definition 2.3.

Recall from (3.12) the notation $d(v, w)=\mathbf{h}_{v}+\mathbf{h}_{w}-2 \min _{\llbracket v, w \rrbracket} \mathbf{h}$, for all $v, w \in t$, that is a pseudo-metric on $t$. We denote by $v \sim w$ the equivalence relation relation $d(v, w)=0$ and as in (3.13), we set for all $u \in S$,

$$
T\left(\mathbf{a}_{u}\right)=\mathbf{a}_{u} / \sim, \quad \operatorname{proj}: \mathbf{a}_{u} \rightarrow T\left(\mathbf{a}_{u}\right) \text {, the canonical projection, } \quad r(u)=\operatorname{proj}(u) .
$$

Note that $T\left(\mathbf{a}_{u}\right)$ only depends on $\left(\mathbf{h}_{v}-I_{u}\right)_{v \in \mathbf{a}_{u}}$ and that $\mathcal{R}\left(\mathbf{a}_{u}\right)$ only depends on $\left(\mathbf{h}_{v}-\right.$ $\left.I_{u} ; U_{v}\right)_{v \in \mathbf{a}_{u}}$. Let $x \in T\left(\mathbf{a}_{u}\right)$; by (3.7) in Lemma 3.5, for all $v, w \in \mathbf{a}_{u}$ such that $\operatorname{proj}(v)=$ 
$\operatorname{proj}(w)=x$, we get $X_{v}=X_{w}$ and it makes sense to set $Z_{x}^{u}=\theta_{X_{u}} X_{v}$; then, $Z^{u}: T\left(\mathbf{a}_{u}\right) \rightarrow$ $\mathcal{R}\left(\mathbf{a}_{u}\right)$ is an isometry given in (3.14). As in (3.15), for all $x \in T\left(\mathbf{a}_{u}\right) \backslash\{r(u)\}$ we denote by $V_{x}^{u}$ the unique real number of $[0,1]$ such that $Z_{x}^{u}=\overleftarrow{Z}_{x}^{u} *\left(V_{x}^{u}\right)$.

Then by (3.16), for all $u \in S$, conditionally given $\mathbf{h}$, the $V_{x}^{u}, x \in T\left(\mathbf{a}_{u}\right)$ are i.i.d. [0,1]uniform r.v. Since the subsets $\left(\mathbf{a}_{u}\right)_{u \in S}$ are pairwise disjoint and since $\mathcal{R}\left(\mathbf{a}_{u}\right)$ only depends on $\left(\mathbf{h}_{v}-I_{u} ; U_{v}\right)_{v \in \mathbf{a}_{u}}$, the $\left(\mathcal{R}\left(\mathbf{a}_{u}\right)\right)_{u \in S}$ are conditionally independent given $\mathbf{h}$. Thus, conditionally given $\mathbf{h}$, the $V_{x}^{u}, x \in T\left(\mathbf{a}_{u}\right) \backslash\{r(u)\}, u \in S$, are i.i.d. [0,1]-uniform r.v.

Next observe that

$$
\left\{X_{v} ; v \in t_{n}\right\}=\bigcup_{u \in S} X_{u} *\left(\mathcal{R}\left(\mathbf{a}_{u}\right)\right)
$$

Therefore, using (3.21) and noting that $\left(X_{u}\right)_{u \in S}$ only depends on $\mathbf{h}$ and on $\left(U_{\varrho_{\mid l}}^{*}\right)_{l \in \mathbb{Z}_{-}}$ that is independent from $\left(U_{u}\right)_{u \in t}$, we conclude that, conditionally given $\mathbf{h}$ and $\left(X_{u}\right)_{u \in S}$, the $V_{x}^{u}, x \in T\left(\mathbf{a}_{u}\right) \backslash\{r(u)\}, u \in S$, are i.i.d. [0,1]-uniform r.v. From the coupling defined in (3.21) and (3.22) we also derive easily the following.

$$
\left\{X_{v}^{+} ; v \in t_{n}\right\}=\bigcup_{u \in S} X_{u}^{\prime} *\left(\mathcal{R}\left(\mathbf{a}_{u}\right)\right), \text { where for all } u \text { in } S, X_{u}^{\prime}:= \begin{cases}U_{u}^{\prime} & \text { if } u \in \bigcup_{p \in \mathbb{N}} S_{2 p+1} \\ \varnothing & \text { otherwise }\end{cases}
$$

Since the $\left(U_{u}^{\prime}\right)_{u \in t}$ are independent from $\mathbf{h}$ and from $\left(U_{u}\right)_{u \in t}$, conditionally given $\mathbf{h}$ and $\left(U_{u}^{\prime}\right)_{u \in S}$, the r.v. $V_{x}^{u}, x \in T\left(\mathbf{a}_{u}\right) \backslash\{r(u)\}, u \in S$, are i.i.d. [0,1]-uniform. Denote by $\mathscr{G}$ the sigma-field generated by $\mathbf{h}$ and by $\left(X_{u}, U_{u}^{\prime}\right)_{u \in S}$. Therefore, we have proved the following.

Conditionally given $\mathscr{G}$, the $V_{x}^{u}, x \in T\left(\mathbf{a}_{u}\right) \backslash\{r(u)\}, u \in S$, are i.i.d. [0,1]-uniform r.v.

Then we set

$$
\forall u \in S, \quad \mathrm{R}_{u}=\Phi_{\mathbf{b}}\left(\mathcal{R}\left(\mathbf{a}_{u}\right)\right) \quad \text { and } \quad \forall c \in \mathbb{N}, \quad M_{c}(n)=\sum_{u \in S} \#\left\{x \in T\left(\mathbf{a}_{u}\right): d(r(u), x) \leq c\right\} .
$$

Note that $M_{c}(n) \leq \# t_{n} \leq \# t$. By (3.25), conditionally given $\mathscr{G}$ we can apply Lemma 3.9 to $\mathrm{T}_{u}:=T\left(\mathbf{a}_{u}\right)$ and $w_{u}:=\Phi_{\mathrm{b}}\left(X_{u}\right), u \in S$, to get an event $A_{c}(n)$ such that $\mathbf{P}\left(A_{c}(n)\right) \leq \mathrm{b}^{-c}(\# t)^{2}$ and such that on $\Omega \backslash A_{c}(n)$,

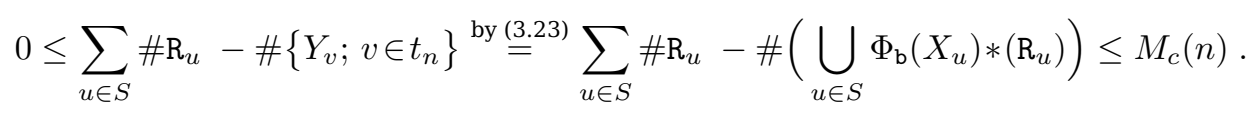

Similarly, conditionally given $\mathscr{G}$, we apply Lemma 3.9 to $\mathrm{T}_{u}:=T\left(\mathbf{a}_{u}\right)$ and to $w_{u}:=\Phi_{\mathrm{b}}\left(X_{u}^{\prime}\right)$, $u \in S$, to get an event $A_{c}^{\prime}(n)$ such that $\mathbf{P}\left(A_{c}^{\prime}(n)\right) \leq \mathrm{b}^{-c}(\# t)^{2}$ and such that on $\Omega \backslash A_{c}^{\prime}(n)$,

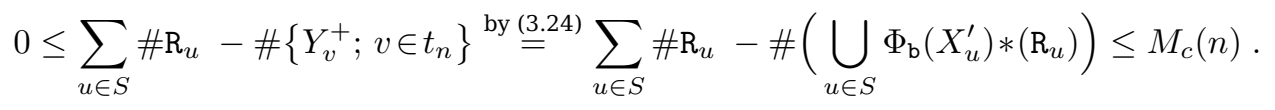

Then, we set $B_{c}(n)=A_{c}(n) \cup A_{c}^{\prime}(n)$; thus, $\mathbf{P}\left(B_{c}(n)\right) \leq 2 \mathrm{~b}^{-c}(\# t)^{2}$ and on $\Omega \backslash B_{c}(n)$, we get $\left|\#\left\{Y_{v} ; v \in t_{n}\right\}-\#\left\{Y_{v}^{+} ; v \in t_{n}\right\}\right| \leq M_{c}(n)$. Now observe that if $x \in T\left(\mathbf{a}_{u}\right)$, then $\mathbf{h}_{x}^{+}=\left|X_{x}^{+}\right|=\left|X_{u}^{\prime}\right|+\left|Z_{x}^{u}\right| \leq 1+d(r(u), x)$. This implies $M_{c}(n) \leq \#\left\{v \in t ;\left|Y_{v}^{+}\right| \leq c+1\right\}$. Thus, on $\Omega \backslash B_{c}(n)$, we get

$$
\left|\#\left\{Y_{v} ; v \in t_{n}\right\}-\#\left\{Y_{v}^{+} ; v \in t_{n}\right\}\right| \leq \#\left\{v \in t ;\left|Y_{v}^{+}\right| \leq c+1\right\} .
$$

We completes the proof of Proposition 3.8 by taking $B_{c}=\bigcup_{0 \leq n<\# t} B_{c}(n)$. 


\subsection{Estimates}

The goal of this section is to establish Proposition 3.16 below. To that end, we first state preliminary estimates. Recall that $\mathbb{W}_{\mathrm{b}}^{*}=\left\{x \in \overline{\mathrm{W}}_{\mathrm{b}}:|x|_{-}=-\infty\right\}$. We denote by $\left(Y_{n}\right)_{n \in \mathbb{N}}$ the canonical process on the space $\left(\mathbb{W}_{\mathrm{b}}^{*}\right)^{\mathbb{N}}$ equipped with product topology (that is Polish) and with the corresponding Borel sigma-field. In this section, let us denote by $P_{\mathrm{o}}$ (instead of $Q_{\mathrm{o}, \mathbb{N}}^{\mathrm{b}}$ ) the law of a Markov chain on $\mathbb{W}_{\mathrm{b}}^{*}$ with transition kernel $p_{\mathrm{b}}$ as defined in (3.3) and whose initial position is o. The following result only contains some standard estimates.

Lemma 3.12. Let $\mathrm{o} \in \mathbb{W}_{\mathrm{b}}^{*}$ be such that $|\mathrm{o}|=0$. For all $k, p \in \mathbb{N}$, we set

$$
\mathrm{o}(p)=\mathrm{o}_{\mid]-\infty,-p]}, \quad \mathrm{Sp}=\{\mathrm{o}(p) ; p \in \mathbb{N}\}, \quad \mathbf{r}_{0}=0 \text { and } \mathbf{r}_{k+1}=\inf \left\{n>\mathbf{r}_{k}: Y_{n} \in \mathrm{Sp}\right\} .
$$

The following holds true.

(i) $P_{\mathrm{o}}$-almost surely for all $k \in \mathbb{N}, \mathbf{r}_{k}<\infty$ and there exists $Z_{k} \in \mathbb{N}$ such that $\mathrm{o}\left(Z_{k}\right)=Y_{\mathbf{r}_{k}}$. Moreover, $\left(Z_{k}\right)_{k \in \mathbb{N}}$ is an $\mathbb{N}$-valued birth-and-death Markov chain whose transition probabilities $(\rho(p, q))_{p, q \in \mathbb{N}}$ are given as follows: for all $p \in \mathbb{N}^{*}$,

$$
\rho(p, p+1)=\frac{1}{2}, \quad \rho(p, p-1)=\frac{1}{2 \mathrm{~b}}, \quad \rho(p, p)=\frac{\mathrm{b}-1}{2 \mathrm{~b}},
$$

and $\rho(0,0)=\rho(0,1)=1 / 2$. Then, $Z$ is transient which implies that almost surely $\left|\mathrm{o} \wedge Y_{n}\right| \rightarrow-\infty$ and that $\left(Y_{n}\right)_{n \in \mathbb{N}}$ is transient under $P_{\mathrm{o}}$.

(ii) For all $x, y \in \mathbb{W}_{\mathrm{b}}^{*}$, we $\operatorname{set} G_{\mathrm{b}}(x, y)=\sum_{n \in \mathbb{N}} P_{x}\left(Y_{n}=y\right)$. Then, for all $p \in \mathbb{N}$,

$$
G_{\mathrm{b}}(x, y)=\frac{2 \mathrm{~b}}{\mathrm{~b}-1} \mathrm{~b}^{|x \wedge y|-|y|} .
$$

(iii) For all $y \in \mathbb{W}_{\mathbf{b}}^{*}$, we set $\mathrm{H}_{y}=\inf \left\{n \in \mathbb{N}: Y_{n}=y\right\}$, with the convention that $\inf \emptyset=\infty$. For all $s \in[0, \infty)$, we set $g(s)=-\log \left(1-\sqrt{1-e^{-2 s}}\right)$. Then, for all $x \in \mathbb{W}_{\mathbf{b}}^{*}, E_{x}\left[e^{-s\left(1+\mathrm{H}_{\overleftarrow{x}}\right)}\right]=$ $\exp (-g(s))$. Moreover, there exists a constant $C \in(1, \infty)$ such that

$$
P_{\mathrm{o}} \text {-a.s. for all } k \in \mathbb{N} \text { and for all } s \in[0,1], \quad E_{\mathrm{o}}\left[e^{-s\left(\mathbf{r}_{k+1}-\mathbf{r}_{k}\right)} \mid Z\right] \geq e^{-C \sqrt{s}} .
$$

Proof. Since under $P_{0},\left(\left|Y_{n}\right|\right)_{n \in \mathbb{N}}$ is distributed as a simple symmetric random walk on $\mathbb{Z}$, we easily see that $P_{\mathrm{o}}$-a.s. for all $k \in \mathbb{N}, \mathbf{r}_{k}<\infty$. The strong Markov property at the stopping times $\mathbf{r}_{k}$ implies that $\left(Z_{k}\right)_{k \in \mathbb{N}}$ is an $\mathbb{N}$-valued birth-and-death Markov chain whose transition probabilities $\rho(p, q)$ are given by (3.27) which easily implies that $Z_{k} \rightarrow \infty$ which entails $(i)$.

Let us prove (ii). For all $x \in \mathbb{W}_{\mathrm{b}}^{*}$, we introduce the stopping times $\mathrm{H}_{x}=\inf \{n \in \mathbb{N}$ : $\left.Y_{n}=x\right\}$ and $\mathrm{H}_{x}^{\circ}=\inf \left\{n \in \mathbb{N}^{*}: Y_{n}=x\right\}$. First observe that for all $x, y \in \mathbb{W}_{\mathrm{b}}^{*}, P_{x}\left(\mathrm{H}_{y}<\infty\right)=$ $P_{x \wedge y}\left(\mathrm{H}_{y}<\infty\right)$. By adapting the argument of $(i)$, the height of the process $Y$ restricted to $\rrbracket-\infty, y \rrbracket$ is a birth-and-death process with transition $\rho(\cdot, \cdot)$ and the Gambler's ruin estimate implies that $P_{x \wedge y}\left(\mathrm{H}_{y}<\infty\right)=\mathrm{b}|x \wedge y|-|y|$. Similarly, we get $P_{y}\left(\mathrm{H}_{y}^{\circ}=\infty\right)=(\mathrm{b}-1) / 2 \mathrm{~b}$. The Markov property at resp. $\mathrm{H}_{y}$ and $\mathrm{H}_{y}^{\circ}$ implies resp. that $G_{\mathrm{b}}(x, y)=P_{x}\left(\mathrm{H}_{y}<\infty\right) G_{\mathrm{b}}(y, y)$ and that $G_{\mathrm{b}}(y, y)=1+P_{y}\left(\mathrm{H}_{y}^{\circ}<\infty\right) G_{\mathrm{b}}(y, y)$, which entails (3.28).

Let us prove $($ iii $)$. Note that under $P_{x},\left(\left|Y_{n}\right|\right)_{n \in \mathbb{N}}$ is distributed as a simple symmetric random walk on $\mathbb{Z}$ with initial position $|x|$. Then $H_{\overleftarrow{x}}$ is the first time $\left(\left|Y_{n}\right|\right)_{n \in \mathbb{N}}$ reaches $|x|-1$; therefore, the law of $\mathrm{H}_{\overleftarrow{x}}$ does not depend on $x$ and we get $E_{x}\left[\exp \left(-s\left(\mathrm{H}_{\overleftarrow{x}}+1\right)\right)\right]=$ $\exp (-g(s))$ by well-known arguments. Next, let us work conditionally given $Z$; we set $x=Y_{\mathbf{r}_{k}+1}$; on the event $\left\{Z_{k}=Z_{k+1}\right\}, \overleftarrow{x}=\mathrm{o}\left(Z_{k}\right)$ and $\mathbf{r}_{k+1}-\mathbf{r}_{k}-1$ is the time at which the shifted random walk $\left(Y_{\mathbf{r}_{k}+1+n}\right)_{n \in \mathbb{N}}$ returns for the first time to $\overleftarrow{x}$; thus, by Markov at $\mathbf{r}_{k}$ and by the previous argument, $P_{\mathrm{o}}$-a.s. on the event $\left\{Z_{k}=Z_{k+1}\right\}, E_{\mathrm{o}}\left[\exp \left(-s\left(\mathbf{r}_{k+1}-\mathbf{r}_{k}\right)\right) \mid Z\right]=$ $\exp (-g(s))$. If $Z_{k} \neq Z_{k+1}$, then $\mathbf{r}_{k+1}-\mathbf{r}_{k}=1$. Consequently, $E_{\mathrm{o}}\left[\exp \left(-s\left(\mathbf{r}_{k+1}-\mathbf{r}_{k}\right)\right) \mid Z\right]=$ $\exp \left(-g(s) \mathbb{1}_{\left\{Z_{k}=Z_{k+1}\right\}}-s \mathbb{1}_{\left\{Z_{k} \neq Z_{k+1}\right\}}\right.$, which implies (3.29) because there exists $C \in(1, \infty)$ such that $g(s) \leq C \sqrt{s}$ for all $s \in[0,1]$ and since $s \leq \sqrt{s}$ for all $s \in[0,1]$. 
Proposition 3.13. Let $\mathrm{o} \in \mathrm{W}_{\mathrm{b}}^{*}$ such that $|\mathrm{o}|=0$. Recall that $P_{\mathrm{o}}$ stands for the canonical law of $\mathrm{W}_{\mathrm{b}}^{*}$-valued Markov chains with transition kernel $p_{\mathrm{b}}$ defined by (3.3) and with initial position o. Let $f:[0, \infty) \rightarrow[0,1]$ be such that $\sum_{p \in \mathbb{N}} \sqrt{f(p)}<\infty$.

$$
P_{\mathrm{o}} \text {-a.s. } \sum_{n \in \mathbb{N}} f\left(-\left|\mathrm{o} \wedge Y_{n}\right|\right)<\infty .
$$

Proof. Recall from (3.26) in Lemma 3.12 notations $o(p)$, Sp and $\mathbf{r}_{k}$ and recall from Lemma $3.12(i)$ the definition of the $\mathbb{N}$-valued birth-and-death process $\left(Z_{k}\right)_{k \in \mathbb{N}}$. Let $\ell \in \mathbb{N}^{*}$. First observe that

$$
W_{\ell}:=\sum_{0 \leq n<\mathbf{r}_{\ell}} f\left(-\left|\mathrm{o} \wedge Y_{n}\right|\right)=\sum_{0 \leq k<\ell} \sum_{\mathbf{r}_{k} \leq n<\mathbf{r}_{k+1}} f\left(Z_{k}\right)=\sum_{0 \leq k<\ell}\left(\mathbf{r}_{k+1}-\mathbf{r}_{k}\right) f\left(Z_{k}\right) .
$$

By the Markov property, the sequence of random times $\mathbf{r}_{k+1}-\mathbf{r}_{k}$ are conditionally independent given $Z$. Thus by (3.29) in Lemma 3.12 (iii), there is $C \in(1, \infty)$ such that for all $s \in[0,1]$,

$$
\begin{aligned}
E_{\mathrm{o}}\left[e^{-s W_{\ell}} \mid Z\right] & =\prod_{0 \leq k<\ell} \mathbf{E}\left[e^{-s\left(\mathbf{r}_{k+1}-\mathbf{r}_{k}\right) f\left(Z_{k}\right)} \mid Z\right] \geq \exp \left(-C \sum_{0 \leq k<\ell} \sqrt{s f\left(Z_{k}\right)}\right) \\
& \geq \exp \left(-C \sqrt{s} \sum_{p \in \mathbb{N}} N_{p} \sqrt{f(p)}\right), \text { where } N_{p}=\#\left\{k \in \mathbb{N}: Z_{k}=p\right\} .
\end{aligned}
$$

Next observe that $E_{\mathrm{o}}\left[N_{p}\right]=G_{\mathrm{b}}(\mathrm{o}, \mathrm{o}(p))=2 \mathrm{~b} /(\mathrm{b}-1)$ by (3.28) in Lemma 3.12 (ii). Set $W=\sum_{n \in \mathbb{N}} f\left(-\left|\mathrm{o} \wedge Y_{n}\right|\right)$; by letting $\ell \rightarrow \infty$ in (3.30) and by Jensen's inequality, we get

$$
E_{\mathrm{o}}\left[e^{-s W}\right] \geq \exp \left(-C \sqrt{s} \sum_{p \in \mathbb{N}} \mathbf{E}\left[N_{p}\right] \sqrt{f(p)}\right)=\exp \left(-C^{\prime} \sqrt{s} \sum_{p \in \mathbb{N}} \sqrt{f(p)}\right),
$$

where $C^{\prime}=2 \mathrm{~b} C /(\mathrm{b}-1)$. Since $\sum_{p \in \mathbb{N}} \sqrt{f(p)}<\infty$, we get $\lim _{s \rightarrow 0+} \mathbf{E}\left[e^{-s W}\right]=1$ which entails that $P_{\mathrm{o}}$-a.s. $W<\infty$. This completes the proof of the proposition.

Lemma 3.14. Let $\mu$ be a probability measure on $\mathbb{N}$ such that $\sum_{k \in \mathbb{N}} k \mu(k)=1$. Let $\boldsymbol{\tau}=(\tau, \varnothing)$ be a random pointed tree such that a.s. $|\tau|_{-}=0, k_{\varnothing}(\tau)=1$ and such that $\theta_{(1)} \tau$ is a $G W(\mu)$-tree. Let $x \in \mathbb{W}_{\mathrm{b}}^{*}$ and let $\Theta=\left(\tau, \varnothing ;\left(X_{v}\right)_{v \in \tau}\right)$ be a random $\mathbb{W}_{\mathrm{b}}^{*}$-valued branching random walk that has law $Q_{x, \tau}^{b}$ conditionally given $\tau$, as defined in Definition 3.1 (iii). For all $y \in \mathbb{W}_{\mathrm{b}}^{*}$ we set

$$
\xi(x, y)=\mathbf{P}\left(y \notin\left\{X_{v} ; v \in \tau \backslash\{\varnothing\}\right\}\right),
$$

which turns out to be strictly positive. Then, we get $1-\xi(x, y) \leq G_{\mathrm{b}}(x, y)=\frac{2 \mathrm{~b}}{\mathrm{~b}-1} \mathrm{~b}|x \wedge y|-|y|$.

Proof. By a simple union bound we first get the following.

$$
\mathbf{P}\left(y \in\left\{X_{v} ; v \in \tau \backslash\{\varnothing\}\right\} \mid \boldsymbol{\tau}\right) \leq \mathbf{E}\left[\sum_{v \in \tau \backslash\{\varnothing\}} \mathbb{1}_{\left\{X_{v}=y\right\}} \mid \boldsymbol{\tau}\right] \leq \sum_{v \in \tau \backslash\{\varnothing\}} Q_{x, \boldsymbol{\tau}}^{\mathrm{b}}\left(X_{v}=y\right) .
$$

By definition of branching random walks, $Q_{x, \tau}^{\mathrm{b}}\left(X_{v}=y\right)=P_{x}\left(Y_{|v|}=y\right)$, where $P_{x}$ stands for the canonical law of the random walk that starts at $x$ in $\mathrm{W}_{\mathrm{b}}^{*}$ and whose transition kernel is $p_{\mathrm{b}}$ as defined in (3.3) in Definition 3.1 (iii). Thus, $\mathbf{P}\left(y \in\left\{X_{v} ; v \in \tau \backslash\{\varnothing\}\right\}\right) \leq$ $\sum_{n \geq 1} \mathbf{E}\left[N_{n}\right] P_{x}\left(Y_{n}=y\right)$, where $N_{n}=\#\{v \in \tau:|v|=n\}$. Since the offspring distribution $\mu$ is critical, $\mathbf{E}\left[N_{n}\right]=1$. Therefore, recalling Lemma (3.12) (ii), we have that the right member is smaller or equal to $G_{\mathrm{b}}(x, y)$, which concludes the proof.

Lemma 3.15. Let $\mu$ be a probability measure on $\mathbb{N}$ such that $\sum_{k \in \mathbb{N}} k \mu(k)=1$. Let us suppose that there exists $\beta \in(0, \infty)$ such that $\sum_{k \in \mathbb{N}^{*}} \mu(k) k(\log k)^{1+\beta}<\infty$. For all $r \in[0,1]$, we set $\psi(r)=\sum_{k \in \mathbb{N}} r^{k} \bar{\mu}(k+1)$, where we recall that for all $k \in \mathbb{N} \backslash\{0\}, \bar{\mu}(k)$ stands for $\sum_{\ell \geq k} \mu(\ell)$. Then, there exists $C_{\beta, \mu} \in(0, \infty)$ such that

$$
\forall r \in(0,1 / 2), \quad 0 \leq 1-\psi(1-r) \leq \frac{C_{\beta, \mu}}{(\log 1 / r)^{1+\beta}} .
$$


Proof. Let $Z$ be an $\mathbb{N} \backslash\{0\}$-valued random variable distributed according to $\bar{\mu}$. Observe that $C:=\mathbf{E}\left[(\log Z)^{1+\beta}\right]=\sum_{l \geq k \geq 1} \mu(l)(\log k)^{1+\beta} \leq \sum_{k \geq 1} \mu(k) k(\log k)^{1+\beta}<\infty$. Then, for all $t>0$ and for all $r \in(0,1 / 2)$, we get

$$
\begin{aligned}
1-\psi(1-r) & =\mathbf{E}\left[\left(1-(1-r)^{Z-1}\right) \mathbb{1}_{\{Z \leq t+1\}}\right]+\mathbf{E}\left[\left(1-(1-r)^{Z-1}\right) \mathbb{1}_{\{Z>t+1\}}\right] \\
& \leq 1-(1-r)^{t}+\mathbf{P}(Z>t+1) \leq 2 r t+\frac{\mathbf{E}\left[(\log Z)^{1+\beta}\right]}{(\log (1+t))^{1+\beta}}=2 r t+C(\log (1+t))^{-1-\beta} .
\end{aligned}
$$

We choose $1+t=r^{-1}(\log 1 / r)^{-1-\beta}$ and we easily get (3.32).

Proposition 3.16. Let $\mu$ be a probability measure on $\mathbb{N}$ such that

$$
\sum_{k \in \mathbb{N}} k \mu(k)=1 \text { and } \exists \beta \in(1, \infty) \text { such that } \sum_{k \in \mathbb{N}} k(\log k)^{1+\beta} \mu(k)<\infty .
$$

Let $\mathrm{o} \in \mathrm{W}_{\mathrm{b}}^{*}$ be such that $|\mathrm{o}|=0$ and let $\Theta:=\left(\tau^{*}, \varrho ;\left(X_{v}\right)_{v \in \tau^{*}}\right)$ be a $\overline{\mathrm{W}}_{\mathrm{b}}$-valued branching random walk whose distribution is the following: $\tau^{*}=\left(\tau^{*}, \varrho\right)$ is an $I P G W^{+}(\mu)$-tree as in Definition 2.8 (ii) and conditionally given $\tau^{*}, \Theta$ has law $Q_{\mathrm{o}, \tau^{*}}^{\mathrm{b}}$ as in Definition 3.1 (iii). Then, with positive probability $\left(X_{v}\right)_{v \in \tau}$ visits its initial position only once. Namely,

$$
\kappa_{\mu, \mathrm{b}}:=\mathbf{P}\left(\mathrm{o} \notin\left\{X_{v} ; v \in \tau^{*} \backslash\{\varrho\}\right\}\right)>0 .
$$

Proof. Let us recall the following notation

$\forall p \in \mathbb{N}, \varrho(p)=\varrho_{\mid]-\infty,-p]}, \quad Y_{p}=X_{\varrho(p)}, \quad \mathrm{Sp}=\{\varrho(p) ; p \in \mathbb{N}\}$ and $\partial \mathrm{Sp}=\left\{u \in \tau^{*} \backslash \mathrm{Sp}: \overleftarrow{u} \in \mathrm{Sp}\right\}$.

By Definition 2.8 (ii) of the right part of an infinite pointed GW $(\mu)$-tree, the subtrees $\left(\theta_{u} \tau^{*}\right)_{u \in \partial \text { Sp }}$ are i.i.d. GW $(\mu)$-trees and the r.v. $\left(k_{\varrho(p)}\left(\tau^{*}\right)\right)_{p \in \mathbb{N}}$ are independent, $k_{\varrho}\left(\tau^{*}\right)$ has law $\mu$ and for all $p \geq 1, k_{\varrho(p)}\left(\tau^{*}\right)$ has law $\bar{\mu}$ that is defined by $\bar{\mu}(k)=\sum_{l \geq k} \mu(l), k \in \mathbb{N}^{*}$ and $\bar{\mu}(0)=0$. For all $u \in \partial \mathrm{Sp}$, we define $\Theta_{u}:=\left(\tau_{u} ; \varnothing ;\left(X_{v}^{u}\right)_{v \in \tau_{u}}\right)$ as follows:

$-\tau_{u}$ is the unique tree such that $k_{\varnothing}\left(\tau_{u}\right)=1$ and $\theta_{(1)} \tau_{u}=\theta_{u} \tau^{*}$ (see (2.8) for the definition of $\left.\theta_{u} \tau^{*}\right)$.

- Since $\overleftarrow{u} \in \mathrm{Sp}$, there exists $p \in \mathbb{N}$ such that $\overleftarrow{u}=\varrho(p)$ and we set $X_{\varnothing}^{u}=Y_{p}$.

- For all $v \in \theta_{u} \tau, X_{(1) * v}^{u}=X_{u * v}$ (recall from (2.4) the definition of the concatenation $u * v$ ). Namely, $\Theta_{u}$ is the restriction of the branching walk $\Theta$ to the subtree stemming from $u$, including the spatial position of $\overleftarrow{u}$. Conditionally given $Y=\left(Y_{p}\right)_{p \in \mathbb{N}}$ and $\left(k_{\varrho(p)}\left(\tau^{*}\right)\right)_{p \in \mathbb{N}}$. the branching random walks $\Theta_{u}, u \in \partial \mathrm{Sp}$, are independent; moreover, conditionally given $\left(Y_{p}, \boldsymbol{\tau}_{u}\right), \Theta_{u}$ has law $Q_{Y_{p}, \tau_{u}}^{d}$; here $\boldsymbol{\tau}_{u}$ stands for $\left(\tau_{u}, \varnothing\right)$ and $p$ is such that $\overleftarrow{u}=\varrho(p)$. Then, for all $q \in \mathbb{N}^{*}$, we set

$$
A_{q}=\left\{0 \notin\left\{X_{v} ; v \in \tau^{*} \backslash\{\varrho\}:|v \wedge \varrho| \geq-q\right\}\right\} \quad \text { and } \mathrm{H}_{\mathrm{o}}^{\circ}=\inf \left\{p \geq 1: Y_{p}=0\right\} .
$$

The event $A_{q}$ decreases as $q \rightarrow \infty$ to the event that the branching walk $\left(X_{v}\right)_{v \in \tau^{*}}$ only visits o once. The previous independence properties then imply the following.

$$
\begin{aligned}
\mathbf{P}\left(A_{q} \mid Y, \tau^{*}\right) & =\mathbb{1}_{\left\{\mathrm{H}_{\circ}^{\circ}>q\right\}} \prod_{u \in \partial \mathrm{Sp}:|u|>-q} Q_{Y_{|\overleftarrow{u}|}^{\mathrm{b}}, \tau_{u}}^{\mathrm{b}}\left(\mathrm{o} \notin\left\{X_{v}^{u} ; v \in \tau_{u} \backslash\{\varnothing\}\right\}\right) \\
\text { and thus } \mathbf{P}\left(A_{q} \mid Y, \mathrm{Sp} \cup \partial \mathrm{Sp}\right) & =\mathbb{1}_{\left\{\mathrm{H}_{\mathrm{o}}^{\circ}>q\right\}} \prod_{u \in \partial \mathrm{Sp}:|u|>-q} \xi\left(Y_{|\overleftarrow{u}|}, \mathrm{o}\right),
\end{aligned}
$$

where we recall the notation $\xi(x, y)$ from (3.31) in Lemma 3.14. Since $Y$ and the r.v. $k_{\varrho(p)}\left(\tau^{*}\right)$ are independent, we get

$$
\begin{aligned}
\mathbf{P}\left(A_{q} \mid Y\right) & =\mathbb{1}_{\left\{\mathrm{H}_{\mathrm{o}}^{\circ}>q\right\}} \mathbf{E}\left[\xi(\mathrm{o}, \mathrm{o})^{k_{\varrho}\left(\tau^{*}\right)}\right] \prod_{1 \leq p \leq q} \mathbf{E}\left[\xi\left(Y_{p}, \mathrm{o}\right)^{k_{\varrho(p)}\left(\tau^{*}\right)-1} \mid Y\right] \\
& =\mathbb{1}_{\left\{\mathrm{H}_{\mathrm{o}}^{\circ}>q\right\}} \phi(\xi(\mathrm{o}, \mathrm{o})) \prod_{1 \leq p \leq q} \psi\left(\xi\left(Y_{p}, \mathrm{o}\right)\right),
\end{aligned}
$$


where we have set

$$
\forall r \in[0,1], \quad \phi(r)=\sum_{k \in \mathbb{N}} r^{k} \mu(k) \quad \text { and } \quad \psi(r)=\sum_{k \in \mathbb{N}} r^{k} \bar{\mu}(k+1)=\frac{1-\phi(r)}{1-r} .
$$

Notice that as $q \rightarrow \infty$, the event $\left\{\mathrm{H}_{\mathrm{O}}^{o}>q\right\}$ decreases to the event $\left\{\mathrm{H}_{\mathrm{O}}^{o}=\infty\right\}$, which has strictly positive probability by Lemma $3.12(i)$. Then, we easily see that (3.34) holds true if

$$
\sum_{p \in \mathbb{N}}\left(1-\psi\left(\xi\left(Y_{p}, o\right)\right)\right)<\infty .
$$

But by Lemma 3.14, $1-\xi\left(Y_{p}, \mathrm{o}\right) \leq \frac{2 \mathrm{~b}}{\mathrm{~b}-1} \mathrm{~b}^{\left|Y_{p} \wedge \mathrm{o}\right|}$ since $|\mathrm{o}|=0$. By (3.32) in Lemma 3.15, there are two constants $C, C^{\prime} \in(0, \infty)$ such that if $-\left|Y_{p} \wedge \mathrm{o}\right| \geq C$, we get

$$
1-\psi\left(\xi\left(Y_{p}, \mathrm{o}\right)\right) \leq \frac{C_{\beta, \mu}}{\left(\log \left(\frac{\mathrm{b}-1}{2 \mathrm{~b}} \mathrm{~b}^{-\left|Y_{p} \wedge \mathrm{o}\right|}\right)\right)^{1+\beta}} \leq \frac{C^{\prime}}{\left(1-\left|Y_{p} \wedge \mathrm{o}\right|\right)^{1+\beta}}
$$

By Proposition 3.13 with $f(x)=(1+x)^{-1-\beta}$, if $\beta>1$, then a.s. $\sum_{p>0}\left(1-\mid Y_{p} \wedge \text { o } \mid\right)^{-1-\beta}<\infty$, which implies (3.35) since $\left|Y_{p} \wedge \mathrm{o}\right| \rightarrow-\infty$, as $p \rightarrow \infty$ as stated in Lemma $3.12(i)$. This completes the proof of the proposition.

We conclude this section with a general estimate for recurrent biased random walks on a (deterministic) rooted ordered tree $T$ that is infinite. More precisely, we fix $\lambda \in(1, \infty)$ and we denote by $\left(Y_{n}\right)_{n \in \mathbb{N}}$ the $\lambda$-biased RW whose transition probabilities are given for all $x, y \in T$ by

$$
\mathbf{P}\left(Y_{n+1}=y \mid Y_{n}=x\right)= \begin{cases}\frac{\lambda}{\lambda+k_{x}(T)} & \text { if } y=\overleftarrow{x} \text { and } x \neq \varnothing \\ \frac{1}{\lambda+k_{x}(T)} & \text { if } x=\overleftarrow{y} \text { and } x \neq \varnothing \\ \frac{1}{k_{\varnothing}(T)} & \text { if } x=\overleftarrow{y}=\varnothing\end{cases}
$$

and $\mathbf{P}\left(Y_{n+1}=y \mid Y_{n}=x\right)=0$ otherwise. Here, recall that $k_{x}(T)$ is the number of children of $x$ in $T$. We also recall that $|x|$ is the height of $x$ in $T$, that $x \wedge y$ is the most recent common ancestor of $x$ and $y$ and that $d_{\mathrm{gr}}$ stands for the graph-distance on $T: d_{\mathrm{gr}}(x, y)=$ $|x|+|y|-2|x \wedge y|$.

Lemma 3.17. We keep the above notations. We assume that $Y$ is recurrent. Then, for all $\ell, n_{1}, n_{2} \in \mathbb{N}$ such that $n_{1}<n_{2}$, we get

$$
\mathbf{P}\left(\left|Y_{n_{1}}\right|+\left|Y_{n_{2}}\right|-2 \min _{n_{1} \leq n \leq n_{2}}\left|Y_{n}\right| \geq 2 \ell+d_{\mathrm{gr}}\left(Y_{n_{1}}, Y_{n_{2}}\right)\right) \leq\left(n_{2}-n_{1}\right) \frac{\lambda-1}{\lambda^{\ell}-1} .
$$

Proof. For all $x \in T$, we set $\mathrm{H}_{x}^{\circ}=\inf \left\{n \in \mathbb{N}^{*}: Y_{n}=x\right\}$ and $\pi(x)=\left(\lambda+k_{x}(T)\right) \lambda^{-|x|}$ if $x \neq \varnothing$ and $\pi(\varnothing)=k_{\varnothing}(T)$. Note that $\pi$ is an invariant measure and standard arguments on electrical networks and random walks on graphs imply that for all distinct $x, y \in T, \pi(x) \mathbf{P}\left(\mathrm{H}_{x}^{\circ}<\right.$ $\mathrm{H}_{y}^{\circ} \mid Y_{0}=y$ ) is the effective conductance between $x$ and $y$ (see R. Lyons and Y. Peres [28] p. 25). If $y \in \rrbracket \varnothing, x \rrbracket$, then it easily implies $\mathbf{P}\left(\mathrm{H}_{x}^{\circ}<\mathrm{H}_{\overleftarrow{y}}^{\circ} \mid Y_{0}=y\right)=(\lambda-1) /\left(\lambda^{|x|-|y|+1}-1\right)$. Similarly, $\mathbf{P}\left(\mathrm{H}_{x}^{\circ}<\mathrm{H}_{\varnothing}^{\circ} \mid Y_{0}=\varnothing\right)=k_{\varnothing}(T)^{-1}(\lambda-1) /\left(\lambda^{|x|}-1\right)$. Thus, for all distinct $x, y \in T$ such that $y \in \llbracket \varnothing, x \rrbracket$,

$$
\mathbf{P}\left(\mathrm{H}_{x}^{\circ}<\mathrm{H}_{\overleftarrow{y}}^{\circ} \mid Y_{0}=y\right) \leq(\lambda-1) /\left(\lambda^{|x|-|y|}-1\right) .
$$

We next define the following sequence of stopping times $\left(\sigma_{k}\right)_{k \in \mathbb{N}}$ by setting $\sigma_{0}=0$ and $\sigma_{k+1}=\inf \left\{n>\sigma_{k}:\left|Y_{n}\right| \leq\left(\left|Y_{0}\right|-k-1\right)_{+}\right\}$that are a.s. finite since $Y$ is recurrent. We then consider the (possibly empty) event $A_{k}=\left\{\exists n \in\left\{\sigma_{k}, \ldots, \sigma_{k+1}-1\right\}: Y_{n} \in \llbracket \varnothing, Y_{0} \rrbracket\right.$ and $\left|Y_{n}\right| \geq$ $\left.\ell+\left(\left|Y_{0}\right|-k-1\right)_{+}\right\}$. We fix $z \in T$ such that $|z| \geq \ell+(|z|-k-1)_{+}$, and let $x, y \in \llbracket \varnothing, z \rrbracket$ be such that $|x|=\ell+(|z|-k-1)_{+}$and $|y|=(|z|-k-1)_{+}$. By the strong Markov property 
at time $\sigma_{k}$ and by the previous inequality for hitting times we get $\mathbf{P}\left(A_{k} \cap\left\{Y_{0}=z\right\}\right) \leq$ $(\lambda-1) /\left(\lambda^{\ell}-1\right) \mathbf{P}\left(Y_{0}=z\right)$. Since on $A_{k}$, we have $\left|Y_{0}\right| \geq \ell+\left(\left|Y_{0}\right|-k-1\right)_{+}$, the previous inequality implies $\mathbf{P}\left(A_{k}\right) \leq(\lambda-1) /\left(\lambda^{\ell}-1\right)$.

Next observe that $\left|Y_{0}\right|+\left|Y_{n}\right|-2 \min _{0 \leq m \leq n}\left|Y_{m}\right|-d_{\mathrm{gr}}\left(Y_{0}, Y_{n}\right)=2\left(\left|Y_{0} \wedge Y_{n}\right|-\min _{0 \leq m \leq n}\left|Y_{m}\right|\right)$ and that

$$
\left\{\left|Y_{0} \wedge Y_{n}\right|-\min _{0 \leq m \leq n}\left|Y_{m}\right| \geq \ell\right\} \subset A_{0} \cup A_{1} \cup \ldots \cup A_{n-1} .
$$

Thus, $\mathbf{P}\left(\left|Y_{0}\right|+\left|Y_{n}\right|-2 \min _{0 \leq m \leq n}\left|Y_{m}\right| \geq 2 \ell+d_{\mathrm{gr}}\left(Y_{0}, Y_{n}\right)\right) \leq n(\lambda-1) /\left(\lambda^{\ell}-1\right)$ and we get (3.37) by the Markov property at time $n_{1}$.

\subsection{Law invariance}

In this section, we first define a successor map for $\overline{\mathrm{W}}_{[0,1]}$-labelled trees that centers the spatial positions and the genealogical tree at the individual coming next in the lexicographical order, generalizing Definition 2.6 for the pointed $\overline{\mathrm{W}}_{[0,1]}$-labelled trees. We then show that free branching random walks are invariant in law under this successor map.

Definition 3.18. Let $\Theta=\left(t, \varrho ; \mathbf{x}=\left(x_{v}\right)_{v \in t}\right)$ be a labelled tree in $\overline{\mathbb{T}}^{\bullet}\left(\overline{\mathbb{W}}_{[0,1]}\right)$ or in $\overline{\mathbb{T}}^{\bullet}\left(\overline{\mathbb{W}}_{\mathrm{b}}\right)$.

(a) We set cent $(\Theta)=\left(\varphi_{|\varrho|}(t), \varphi_{|\varrho|}(\varrho) ;\left(\varphi_{\left|x_{\ell}\right|}\left(x_{\varphi_{-|\varrho|}(v)}\right)\right)_{v \in \varphi_{|\varrho|}(t)}\right)$. We keep calling cent $(\cdot)$ the centering map. As already mentioned, the spatial marks are also shifted.

(b) We next set $\operatorname{scc}(\Theta)=\operatorname{cent}(t, \operatorname{scc}(\varrho) ; \mathbf{x})$ where we recall that $\operatorname{scc}(\varrho)$ stands for the vertex of $t$ coming next in the lexicographical order as defined by (2.7). We keep calling $\operatorname{scc}(\cdot)$ the successor map.

(c) Recall from Definition $2.6(c)$ the right-part $[(t, \varrho)]^{+}$of $(t, \varrho)$. To simplify the notation, we set $\left(t^{\prime}, \varrho^{\prime}\right)=[(t, \varrho)]^{+}$and we recall from Definition $3.18(c)$ that there exists a unique one-to-one map $\psi: t^{\prime} \rightarrow\left\{\varrho_{\mid p} ; p \leq|\varrho|\right\} \cup\left\{v \in t: \varrho<_{t} v\right\}$ such that $\psi\left(\varrho^{\prime}\right)=$ $\varrho$ that preserves the relative height and that is increasing with respect to the lexicographical order; then, we set $[\Theta]^{+}=\left(t^{\prime}, \varrho^{\prime}, \mathbf{x}^{\prime}=\left(x_{v}^{\prime}\right)_{v \in t^{\prime}}\right)$ where $x_{v}^{\prime}=x_{\psi(v)}$, for all $v \in t^{\prime}$. We keep calling $[\Theta]^{+}$the right-part of $\Theta$.

(d) We set $\operatorname{scc}^{+}(\Theta)=[\operatorname{scc}(\Theta)]^{+}$; we keep calling $\mathrm{scc}^{+}(\cdot)$ the right-successor map.

We next explain a way to generate a free branching random walk from an i.i.d. field that is suited to the successor map. To that end we fix $\mathbf{t}=(t, u) \in \overline{\mathbb{T}}^{\bullet}$ such that $|t|_{-}=-\infty$. Let $\mathbf{o}$ be a $\mathbb{Z}_{-}$-indexed sequence of mutually independent uniform r.v. on $[0,1]$. Let $\Theta$ be a $\overline{\mathbb{W}}_{[0,1]}$-valued branching random walk that has conditional law $Q_{\mathbf{o}, \mathbf{t}}$ given $\mathbf{o}$ as in Definition $3.1(i v)$. To simplify we denote by $\mathbf{Q}_{\mathbf{t}}$ the (unconditional) law of $\boldsymbol{\Theta}$. Let $\varepsilon_{v}, U_{v}, v \in t$ be independent r.v. such that $U_{v}$ is uniformly distributed on $[0,1]$ and $\mathbf{P}\left(\varepsilon_{v}=1\right)=\mathbf{P}\left(\varepsilon_{v}=-1\right)=1 / 2$. From the field of i.i.d. r.v. $\boldsymbol{\Theta}_{0}:=\left(t, u ;\left(\varepsilon_{v}, U_{v}\right)_{v \in t}\right)$, we now explain how to construct a branching random walk that has law $\mathbf{Q}_{\mathbf{t}}$.

Recall that $\llbracket v, w \rrbracket$ stands for the shortest path (with respect to the graph-distance) that joins $v$ and $w$ in $t$ (along with the previous notation $\llbracket v, w \llbracket, \rrbracket v, w \rrbracket$ and $\rrbracket v, w \llbracket$, introduced at the beginning of Section 3.2). Recall also that $\rrbracket-\infty, v \rrbracket$ stands for the lineage of $v$ : namely, $\rrbracket-\infty, v \rrbracket=\left\{v_{\mid l} ; l \leq|v|\right\}$. Recall from (2.5) the definition of the most recent common ancestor $v \wedge w$ in $t$. We first set

$$
\forall v \in t, \quad H_{v}=\sum_{w \in \llbracket u, u \wedge v \llbracket}\left(-\varepsilon_{w}\right)+\sum_{w \in \llbracket u \wedge v, v \llbracket} \varepsilon_{w},
$$

with the convention that $H_{u}=0$. Note that $H$ takes arbitrary negative values on the lineage of $v$. Namely $H$ satisfies (3.4). It is easy to see that $\left(t, u ;\left(H_{v}\right)_{v \in t}\right)$ is a $\mathbb{Z}$-valued branching random walk whose spatial motion is that of a simple symmetric random walk on $\mathbb{Z}$ and whose initial position is a.s. $H_{u}=0$. Recall from (3.5) the notation $v(k)$ for all integers $k \leq H_{v}$ : namely, $v(k) \in \rrbracket-\infty, v \rrbracket$ is such that $H_{v(k)}=\min _{w \in \llbracket v(k), v \rrbracket} H_{w}=k>H_{\overleftarrow{v(k)}}$. 
As in (3.6), for all $v \in t$ we set $X_{v}=\left(U_{v(k)}\right)_{k \leq H_{v}}$. We denote $X_{u}$ by o. It shows that there exists a deterministic map $F$ such that

$$
F\left(\boldsymbol{\Theta}_{0}\right):=\left(t, u ;\left(X_{v}\right)_{v \in t}\right) \text {, which has law } \mathbf{Q}_{\mathbf{t}} .
$$

Let $u^{\prime} \in t$ and set $F\left(t, u^{\prime} ;\left(\varepsilon_{v}, U_{v}\right)_{t \in t}\right)=:\left(t, u^{\prime},\left(X_{v}^{\prime}\right)_{v \in t}\right)$. Then, set $H_{v}^{\prime}=\left|X_{v}^{\prime}\right|, v \in t$. We deterministically check that

$$
\forall v \in t, \quad H_{v}^{\prime}-H_{v}=-H_{u^{\prime}} \text { and } X_{v}^{\prime}=\varphi_{\left|X_{u^{\prime}}\right|}\left(X_{v}\right) .
$$

We next define $\left[\Theta_{0}\right]^{+}$as in Definition $3.18(c)$ : to simplify notation, we set $\left(t^{\prime}, u^{\prime}\right)=$ $[(t, u)]^{+}$and we recall from Definition $2.6(c)$ that there exists a unique one-to-one map $\psi: t^{\prime} \rightarrow\left\{u_{\mid p} ; p \leq|u|\right\} \cup\left\{v \in t: u<_{t} v\right\}$ such that $\psi\left(u^{\prime}\right)=u$ that preserves the relative height and that is increasing with respect to the lexicographical order. Then, we simply define $\left[\boldsymbol{\Theta}_{0}\right]^{+}=\left(t^{\prime}, u^{\prime},\left(\varepsilon_{v}^{\prime}, U_{v}^{\prime}\right)_{v \in t^{\prime}}\right)$ where $\left(\varepsilon_{v}^{\prime}, U_{v}^{\prime}\right)=\left(\varepsilon_{\psi(v)}, U_{\psi(v)}\right)$ for all $v \in t^{\prime}$ and also set

$$
\widetilde{\operatorname{scC}^{+}}\left(\Theta_{0}\right)=\left[\operatorname{scc}(t, u) ;\left(\varepsilon_{v}, U_{v}\right)_{v \in t}\right]^{+} .
$$

By (3.39) and since $X_{v}$ is a deterministic function of $\left(\left(\varepsilon_{w}, U_{w}\right), w \in \rrbracket-\infty, u \rrbracket \cup \rrbracket-\infty, v \rrbracket\right)$, it is easy to check deterministically

$$
F\left(\widetilde{\operatorname{scc}}^{+}\left(\Theta_{0}\right)\right)=\operatorname{scc}^{+}\left(F\left(\Theta_{0}\right)\right) .
$$

Then, (3.2), (3.38), (3.39) and (3.41) immediately entail the following lemma.

Lemma 3.19. Let $\mathbf{t}=(t, u) \in \overline{\mathbb{T}}^{\bullet}$. We assume that $|t|_{-}=-\infty$. Let $\boldsymbol{\Theta}=\left(t, u ;\left(X_{v}\right)_{v \in t}\right)$ have law $\mathbf{Q}_{\mathbf{t}}$. Then $\operatorname{scc}(\boldsymbol{\Theta})$ has law $\mathbf{Q}_{\mathbf{s c c}(\mathbf{t})}$ and $\operatorname{scc}^{+}(\boldsymbol{\Theta})$ has law $\mathbf{Q}_{\mathbf{s c c}+(\mathbf{t})}$.

The next result is the key point in the proofs of the various laws of large numbers for the range of branching random walks that we prove in the next section.

Theorem 3.20. Let $\mu$ be a probability measure on $\mathbb{N}$ such that $\sum_{k \in \mathbb{N}} k \mu(k)=1$. Let $\mathbf{o}:=\left(U_{k}\right)_{k \in \mathbb{Z}_{-}}$be a sequence of independent uniform r.v. on $\{1, \ldots, \mathrm{b}\}$. Let $\Theta=$ $\left(\tau^{*}, \varrho ;\left(X_{v}\right)_{v \in \tau^{*}}\right)$ be a $\overline{\mathrm{W}}_{\mathrm{b}}$-valued branching random walk whose distribution is the following: $\tau^{*}=\left(\tau^{*}, \varrho\right)$ is an IPGW $W^{+}(\mu)$-tree as in Definition $2.8(i i)$ and conditionally given $\tau^{*}$ and $\mathbf{o}, \Theta$ has law $Q_{\mathbf{o}, \tau^{*}}^{\mathrm{b}}$ as in Definition 3.1 (iii). Then

$$
\operatorname{scc}^{+}(\Theta) \stackrel{(\text { law) }}{=} \Theta
$$

where we recall that $\mathrm{scc}^{+}$stands for the right-successor map as in Definition $3.18(d)$.

Next, denote by $\left(v_{n}\right)_{n \in \mathbb{N}}$ the sequence of the vertices that are not direct ancestors of $\varrho$ listed in the lexicographical order. Namely, $v_{0}=\varrho, v_{n}<_{\tau^{*}} v_{n+1}$ and $\left\{v_{n} ; n \in \mathbb{N}\right\}=\{v \in \tau$ : $\left.\varrho \leq_{\tau^{*}} v\right\}$. Then,

$$
\mathbf{P} \text {-a.s. } \quad \frac{1}{n} \#\left\{X_{v_{k}} ; 1 \leq k \leq n\right\} \underset{n \rightarrow \infty}{\longrightarrow} c_{\mu, \mathrm{b}}:=\mathbf{P}\left(\mathbf{o} \notin\left\{X_{v_{n}} ; n \geq 1\right\}\right) .
$$

Furthermore, if $\mu$ satisfies (3.33), then $c_{\mu, \mathrm{b}}>0$.

Proof. Let $\boldsymbol{\Theta}_{0}:=\left(\tau^{*}, \varrho ;\left(\varepsilon_{v}, U_{v}\right)_{v \in \tau^{*}}\right)$ be distributed as follows: conditionally given $\boldsymbol{\tau}^{*}, \varepsilon_{v}$, $U_{v}, v \in t$ are independent r.v. such that $U_{v}$ is uniformly distributed on $[0,1]$ and $\varepsilon_{v}$ is uniformly distributed on $\{-1,1\}$. Then, we set $F\left(\boldsymbol{\Theta}_{0}\right)=\boldsymbol{\Theta}^{\prime}=\left(\tau^{*}, \varrho ;\left(X_{v}^{\prime}\right)_{v \in \tau^{*}}\right)$ as in (3.38). Conditionally given $\boldsymbol{\tau}^{*}, \boldsymbol{\Theta}^{\prime}$ has law $\mathbf{Q}_{\tau^{*}}$ and Remarks 3.3 allows to take $\boldsymbol{\Theta}=\Phi_{\mathrm{b}}\left(\boldsymbol{\Theta}^{\prime}\right)$ that has the desired law. Lemma 3.19 combined with proposition 2.10 imply that $\operatorname{scc}^{+}\left(\Theta^{\prime}\right)$ has the same law as $\Theta^{\prime}$ and since obviously $\Phi_{\mathrm{b}}\left(\operatorname{scc}^{+}\left(\Theta^{\prime}\right)\right)=\operatorname{scc}^{+}(\Theta)$, we get (3.42).

For all $n \geq m \geq 0$, we set $R_{m, n}(\boldsymbol{\Theta})=\#\left\{X_{v_{k}} ; m<k \leq n\right\}$ and we denote by $\mathbf{s c c}_{\ell}^{+}$the $\ell$-th iterate of scc ${ }^{+}$. Observe that $R_{m, n}\left(\operatorname{scc}_{\ell}^{+}(\Theta)\right)=R_{m+\ell, n+\ell}(\Theta)$, with an obvious notation. Then, the r.v. $\left(R_{m, n}\left(\boldsymbol{\Theta}^{+}\right)_{m \geq n \geq 1}\right)$ satisfy Assumptions (1.7), (1.8) and (1.9) of Liggett's 
version of Kingman's subadditive ergodic theorem (see Theorem 1.10 in [26] p. 1280) that asserts that there exists a $[0,1]$-valued r.v. $R$ such that $R_{0, n}(\boldsymbol{\Theta}) / n \rightarrow R$ almost surely (and in $L^{1}$ ).

We next prove that $R$ is a.s. constant. Recall from (3.40) the definition of $\widetilde{\operatorname{scc}}^{+}\left(\boldsymbol{\Theta}_{0}\right)$ and we denote by $\widetilde{\mathrm{scc}_{\ell}^{+}}$the $\ell$-th iterate of $\widetilde{\mathrm{scc}}+$ : namely, $\widetilde{\mathrm{scc}_{\ell}^{+}}\left(\boldsymbol{\Theta}_{0}\right)=\left[\left(\tau^{*}, v_{\ell}\right) ;\left(\varepsilon_{v}, U_{v}\right)_{v \in \tau^{*}}\right]^{+}$. Denote by $\mathscr{G}_{\ell}$ the sigma-field generated by $\widetilde{\operatorname{scc}}_{\ell}^{+}\left(\Theta_{0}\right)$. As a consequence of (3.41), $\operatorname{scc}_{\ell}^{+}(\boldsymbol{\Theta})=\Phi_{d}\left(F\left(\widetilde{\operatorname{scc}}_{\ell}^{+}\left(\boldsymbol{\Theta}_{0}\right)\right)\right.$, the r.v. $\left(R_{\ell, \ell+n}(\boldsymbol{\Theta})\right)_{n \in \mathbb{N}}$ are $\mathscr{G}_{\ell}$-measurable. Note that the sigma-fields $\mathscr{G}_{\ell}$ decrease in $\ell$. Next, we set $\mathscr{G}=\bigcap_{\ell \in \mathbb{N}} \mathscr{G}_{\ell}$ that can be viewed as the tail sigma-field of the subtrees grafted on the infinite line of ancestors; since additional marks $\left(\varepsilon_{v}, U_{v}\right)$ are i.i.d., Kolmogorov's zero-one law applies and $\mathscr{G}$ is $\mathbf{P}$-trivial. Furthermore, the subadditivity for the $R_{m, n}$ entail that a.s. $R=\lim _{n \rightarrow \infty} n^{-1} R_{\ell, \ell+n}(\boldsymbol{\Theta})$, for all $\ell$. Thus, $R$ is $\mathscr{G}$-measurable which implies that it is a.s. constant.

Consequently, $R=\lim _{n \rightarrow \infty} \mathbf{E}\left[R_{0, n}(\mathbf{\Theta})\right] / n$. By an elementary argument,

$$
\mathbf{E}\left[R_{0, n}(\boldsymbol{\Theta})\right]=\sum_{1 \leq k \leq n} \mathbf{P}\left(X_{v_{k}} \notin\left\{X_{v_{k+1}}, \ldots, X_{v_{n}}\right\}\right) .
$$

Since the law of $\boldsymbol{\Theta}$ is preserved by the map scc $\operatorname{sc}^{+}(\cdot)$, we get $\mathbf{P}\left(X_{v_{k}} \notin\left\{X_{v_{k+1}}, \ldots, X_{v_{n}}\right\}\right)=$ $\mathbf{P}\left(X_{\varrho} \notin\left\{X_{v_{1}}, \ldots, X_{v_{n-k}}\right\}\right)$ and thus

$$
\frac{1}{n} \mathbf{E}\left[R_{0, n}(\boldsymbol{\Theta})\right]=\frac{1}{n} \sum_{0 \leq k \leq n-1} \mathbf{P}\left(X_{\varrho} \notin\left\{X_{v_{1}}, \ldots, X_{v_{k}}\right\}\right) \underset{n \rightarrow \infty}{\longrightarrow} c_{\mu, \mathbf{b}}
$$

by Cesàro. Finally, observe that $c_{\mu, \mathrm{b}} \geq \kappa_{\mu, \mathrm{b}}$, where $\kappa_{\mu, \mathrm{b}}$ is defined in (3.34) in Proposition 3.16 that completes the proof of the theorem.

\subsection{Proof of Theorem 1.1}

We fix $\gamma \in(1,2]$. Let $\tau$ be a GW( $\mu$-tree whose offspring distribution $\mu$ satisfies $(\mathbf{H})$ in (1.1). We set $\boldsymbol{\tau}=(\tau, \varnothing)$. Let $\mathbf{o}:=\left(U_{k}\right)_{k \in \mathbb{Z}_{-}}$be a sequence of independent uniform r.v. on $\{1, \ldots, \mathrm{b}\}$. Let the $\overline{\mathrm{W}}_{\mathrm{b}}$-valued branching random walk $\boldsymbol{\Theta}_{d}=\left(\tau, \varnothing ;\left(Y_{v}\right)_{v \in \tau}\right)$ have conditional law $Q_{\mathbf{o}, \tau}^{\mathrm{b}}$ given $\tau$ and $\mathbf{o}$ as in Definition 3.1 (iii). We also introduce the $\mathbb{W}_{\mathrm{b}}$-valued branching random walk $\boldsymbol{\Theta}_{\mathrm{b}}^{+}=\left(\tau, \varnothing ;\left(Y_{v}^{+}\right)_{v \in \tau}\right)$ that has conditional law $Q_{\tau}^{+\mathrm{b}}$ given $\tau$ as in Definition $3.1(i)$. For all integers $0 \leq k<\# \tau$, we denote

$$
R_{k}\left(\boldsymbol{\Theta}_{\mathrm{b}}\right)=\#\left\{Y_{v} ; v \in \tau: v \leq_{\tau} v_{k}\right\} \text { and } R_{k}\left(\boldsymbol{\Theta}_{\mathrm{b}}^{+}\right)=\#\left\{Y_{v}^{+} ; v \in \tau: v \leq_{\tau} v_{k}\right\} \text {, }
$$

where $v_{k}$ stands for the $k$-th smallest vertex of $\tau$ with respect to the lexicographical order $\leq_{\tau}$. Arguing as in Le Gall and L. [24], we derive the following result from Theorem 3.20.

Proposition 3.21 (adapted from Theorem 7 [24]). Let $\gamma \in(1,2]$ and let $\mu$ satisfy $(\mathbf{H})$ as in (1.1). We keep the above notation and we recall from (3.43) the definition of $c_{\mu, \mathrm{b}}$ and from (3.44) the definition of $R_{n}\left(\boldsymbol{\Theta}_{\mathrm{b}}\right)$. Then,

$$
\forall \varepsilon \in(0, \infty), \quad \mathbf{P}\left(\sup _{t \in[0,1]} \frac{1}{n}\left|R_{\lfloor n t\rfloor}\left(\boldsymbol{\Theta}_{\mathrm{b}}\right)-c_{\mu, \mathrm{b}} n t\right|>\varepsilon \mid \# \tau=n\right) \underset{n \rightarrow \infty}{\longrightarrow} 0 .
$$

Proof. The proof can be adapted verbatim from the way Proposition 6 and Theorem 7 pp. 284-289 in [24] are deduced from Proposition 3 and Theorem 4 pp. 280-284 in [24]. Here our Theorem 3.20 plays the role of Proposition 3 and Theorem 4 in [24] and we only give a brief sketch of the proof. Following the same arguments as in Proposition 6 in [24], we first prove that for any fixed $s \in[0,1]$,

$$
\forall \varepsilon \in(0, \infty), \quad \mathbf{P}\left(\frac{1}{n}\left|R_{\lfloor n s\rfloor}\left(\mathbf{\Theta}_{\mathrm{b}}\right)-c_{\mu, \mathrm{b}} n s\right|>\varepsilon \mid \# \tau>n\right) \underset{n \rightarrow \infty}{\longrightarrow} 0 .
$$


From this limit and the same absolute continuity argument as in the proof of Theorem 7 of [24], we get for any fixed $s \in[0,1]$,

$$
\forall \varepsilon \in(0, \infty), \quad \mathbf{P}\left(\frac{1}{n}\left|R_{\lfloor n s\rfloor}\left(\boldsymbol{\Theta}_{\mathrm{b}}\right)-c_{\mu, \mathrm{b}} n s\right|>\varepsilon \mid \# \tau=n\right) \underset{n \rightarrow \infty}{\longrightarrow} 0 .
$$

We then get (3.45) thanks to the following variant of the second Dini's theorem.

Lemma 3.22. For all $n \in \mathbb{N}$, let $\left(\Omega_{n}, \mathscr{F}_{n}, \mathbf{P}_{n}\right)$ be a probability space on which a nondecreasing right-continuous process $\left(X_{t}^{(n)}\right)_{t \in[0, \infty)}$ is defined. Let $(x(t))_{t \in[0, \infty)}$ be a (deterministic) continuous function. Suppose that for all $t \in[0, \infty)$, the real valued r.v. $X_{t}^{(n)}$ under $\mathbf{P}_{n}$ tends to $x(t)$ in law. Then $\lim _{n \rightarrow \infty} \mathbf{P}_{n}\left(\sup _{t \in[0, p]}\left|X_{t}^{(n)}-x(t)\right|>\varepsilon\right)=0$ for all $\varepsilon \in(0, \infty)$ and all $p \in \mathbb{N}$.

Proof of Lemma 3.22. We fix $p \in \mathbb{N}$. Set $w_{n, q}=\max _{0 \leq k \leq p 2^{q}}\left|X_{k 2^{-q}}^{(n)}-x\left(k 2^{-q}\right)\right|$ for all $n, q \in$ $\mathbb{N}$. Since the convergence in law to a constant implies the convergence in probability, we easily get for all $q$ that $\lim _{n \rightarrow \infty} \mathbf{P}_{n}\left(w_{n, q}>\varepsilon\right)=0$. Next set $v_{q}=\max \{|x(t)-x(s)| ; s, t \in[0, p]$ : $|s-t| \leq 2^{-q}$ \} that tends to 0 as $q \rightarrow \infty$ since $x$ is continuous. By monotonicity of $X_{t}^{(n)}$, it is then easy to see that for all $t \in[0, p]$, that $\left|X_{t}^{(n)}-x(t)\right| \leq 2 v_{q}+3 w_{n, q}$, which easily implies the desired result.

End of the proof of Theorem 1.1. We keep the notation from above and recall from (3.43) the definition of $c_{\mu, \mathrm{b}}$ and from (3.44) the definition of $R_{n}\left(\boldsymbol{\Theta}_{\mathrm{b}}^{+}\right)$. We now derive Theorem 1.1 from (3.45) and Proposition 3.8 that allows to change the state space from $\overline{\mathrm{W}}_{\mathrm{b}}$ to $\mathrm{W}_{\mathrm{b}}$.

For all positive integers $n$, we set $c_{n}=4 \log _{\mathrm{b}} n, L_{n}=\#\left\{v \in \tau:\left|Y_{v}^{+}\right| \leq c_{n}\right\}, N_{n}=$ $\#\{v \in \tau:|v|=n\}$ and $C_{n}=N_{0}+N_{1}+\ldots+N_{n}$. Let $\left(S_{n}\right)_{n \in \mathbb{N}}$ be a simple symmetric random walk on $\mathbb{Z}$ with initial position $S_{0}=0$. By definition of $\Theta_{\mathrm{b}}^{+}$, for all $v \in \tau$, we get $\mathbf{E}\left[f\left(\left|Y_{v}^{+}\right|\right) \mid \tau\right]=\mathbf{E}\left[f\left(\left|S_{|v|}\right|\right) \mid \tau\right]$. Then, for all $b_{n} \in \mathbb{N}^{*}$ we get

$$
\mathbf{E}\left[L_{n} \mid \tau\right]=\sum_{k \in \mathbb{N}} N_{k} \mathbf{P}\left(\left|S_{k}\right| \leq c_{n}\right) \leq C_{b_{n}}+\sigma\left(b_{n}, \frac{c_{n}}{\sqrt{b_{n}}}\right) \# \tau,
$$

where we have set $\sigma(p, s)=\sup _{k \geq p} \mathbf{P}\left(\left|S_{k}\right| k^{-\frac{1}{2}} \leq s\right)$ for all $p \in \mathbb{N}^{*}$ and all $s \in(0, \infty)$. Observe that any fixed $p, \lim _{s \rightarrow 0+} \sigma(p, s)=0$. We now take $b_{n}=c_{n}^{4}$.

We next claim that for all $\eta \in(0, \infty)$,

$$
\lim _{n \rightarrow \infty} \mathbf{P}\left(\frac{1}{n} C_{b_{n}} \geq \eta \mid \# \tau=n\right)=0 .
$$

Proof of (3.47). For all $\varepsilon \in(0, \infty)$ and all $y \in \mathbb{R}$, we set $f_{\varepsilon}(y)=\left(1-\varepsilon^{-1}(|y|-\varepsilon)_{+}\right)_{+}$that is continuous and such that $\mathbb{1}_{[-\varepsilon, \varepsilon]} \leq f_{\varepsilon} \leq \mathbb{1}_{[-2 \varepsilon, 2 \varepsilon]}$. Recall from the introduction that $\left(H_{k}\right)_{0 \leq k<\# \tau}$ stands for the height process of the tree $\tau$ and recall the convergence (1.4). Then note that

$$
\frac{1}{n} C_{a_{n} \varepsilon} \leq Q_{\varepsilon}^{n}:=\int_{0}^{1} f_{\varepsilon}\left(\frac{1}{a_{n}} H_{\lfloor n s\rfloor}\right) d s .
$$

By (1.4), $Q_{\varepsilon}^{n}$ under $\mathbf{P}(\cdot \mid \# \tau=n)$ tends in law to $Q_{\varepsilon}:=\int_{0}^{1} f_{\varepsilon}\left(H_{s}\right) d s$. We recall here that a.s. for all $s \in(0,1), H_{s}>0$ (see D. [11] or Section 4.4). Thus $\lim _{\varepsilon \rightarrow 0+} Q_{\varepsilon}=0$ and we get for all $\eta \in(0, \infty)$

$$
\begin{aligned}
\limsup _{\varepsilon \rightarrow 0+} \limsup _{n \rightarrow \infty} \mathbf{P}\left(\frac{1}{n} C_{a_{n} \varepsilon} \geq \eta \mid \# \tau=n\right) & \leq \limsup _{\varepsilon \rightarrow 0+} \limsup _{n \rightarrow \infty} \mathbf{P}\left(Q_{\varepsilon}^{n} \geq \eta \mid \# \tau=n\right) \\
& \leq \limsup _{\varepsilon \rightarrow 0+} \mathbf{P}\left(Q_{\varepsilon}^{n} \geq \eta\right)=0 .
\end{aligned}
$$

Since $\left(a_{n}\right)_{n \in \mathbb{N}}$ is a $\frac{\gamma-1}{\gamma}$-regularly varying sequence, $b_{n} / a_{n} \rightarrow 0$ and (3.48) entails (3.47). 
Since $\frac{1}{n} C_{b_{n}} \in(0,1]$, it also implies that $\mathbf{E}\left[\frac{1}{n} C_{b_{n}} \mid \# \tau=n\right] \rightarrow 0$ as $n \rightarrow \infty$. By (3.46) we get $\lim _{n \rightarrow \infty} \mathbf{E}\left[\frac{1}{n} L_{n} \mid \# \tau=n\right]=0$. We next apply Proposition 3.8 to $c=\left\lfloor 4 \log _{\mathrm{b}} n\right\rfloor+1$ : (3.18) implies the following.

$$
\begin{aligned}
& \mathbf{P}\left(\sup _{s \in[0,1]} \frac{1}{n}\left|R_{\lfloor n s\rfloor}\left(\mathbf{\Theta}_{\mathrm{b}}^{+}\right)-c_{\mu, \mathrm{b}} n s\right|>2 \varepsilon \mid \# \tau=n\right) \\
& \quad \leq 2 \mathrm{~b} n^{-1}+\mathbf{P}\left(\frac{1}{n} L_{n}>\varepsilon \mid \# \tau=n\right)+\mathbf{P}\left(\sup _{s \in[0,1]} \frac{1}{n}\left|R_{\lfloor n s\rfloor}\left(\mathbf{\Theta}_{\mathrm{b}}\right)-c_{\mu, \mathrm{b}} n s\right|>\varepsilon \mid \# \tau=n\right),
\end{aligned}
$$

which implies (1.3) in Theorem 1.1 by (3.45) since $\mathbf{E}\left[\frac{1}{n} L_{n} \mid \# \tau=n\right] \rightarrow 0$ as $n \rightarrow \infty$.

\section{Snake metrics and the Brownian cactus}

\subsection{Pseudo-metrics on a closed interval}

Definition 4.1. Let $\zeta \in(0, \infty)$. We introduce the following spaces.

(a) We denote by $\mathbf{C}\left([0, \zeta]^{2}, \mathbb{R}\right)$ the space of continuous functions from $[0, \zeta]^{2}$ to $\mathbb{R}$ that is a Banach space when equipped with uniform norm $\|\cdot\|$.

(b) We denote by $\mathbf{M}([0, \zeta])$ the set of continuous pseudo-metrics on $[0, \zeta]$. Namely, it is the set of $d \in \mathbf{C}\left([0, \zeta]^{2}, \mathbb{R}\right)$ such that for all $s_{1}, s_{2}, s_{3} \in[0, \zeta]$,

$$
d\left(s_{1}, s_{2}\right) \geq 0, d\left(s_{1}, s_{1}\right)=0, d\left(s_{1}, s_{2}\right)=d\left(s_{2}, s_{1}\right) \text { and } d\left(s_{1}, s_{3}\right) \leq d\left(s_{1}, s_{2}\right)+d\left(s_{2}, s_{3}\right) .
$$

(c) We denote by $\mathbf{M T}([0, \zeta])$ the space of continuous pseudo-metrics $d \in \mathbf{M}([0, \zeta])$ such that for all $s_{1}, s_{2}, s_{3}, s_{4} \in[0, \zeta]$,

$$
d\left(s_{1}, s_{2}\right)+d\left(s_{3}, s_{4}\right) \leq \max \left(d\left(s_{1}, s_{3}\right)+d\left(s_{2}, s_{4}\right) ; d\left(s_{1}, s_{4}\right)+d\left(s_{2}, s_{3}\right)\right) .
$$

We call the latter inequality the four points inequality.

We easily check that $\mathbf{M T}([0, \zeta])$ and $\mathbf{M}([0, \zeta])$ are closed subsets of $\left(\mathbf{C}\left([0, \zeta]^{2}, \mathbb{R}\right),\|\cdot\|\right)$. We shall need the following compactness criterion in $\mathbf{M}([0, \zeta])$.

Lemma 4.2. For all $\eta \in(0, \infty)$ and all $d \in \mathbf{M}([0, \zeta])$, we set

$$
q_{\eta}(d)=\max \left\{d\left(s, s^{\prime}\right) ; s, s^{\prime} \in[0, \zeta]:\left|s-s^{\prime}\right| \leq \eta\right\} .
$$

Let $\mathcal{D}$ be a subset of $\mathbf{M}([0, \zeta])$. Then, the closure of $\mathcal{D}$ is compact if and only if $\sup _{d \in \mathcal{D}} q_{\eta}(d) \longrightarrow 0$ as $\eta \rightarrow 0+$.

Proof. For all $f \in \mathbf{C}\left([0, \zeta]^{2}, \mathbb{R}\right)$ and all $\eta \in(0, \infty)$, set

$$
\omega_{\eta}(f)=\max \left\{\left|f\left(s_{1}, s_{1}^{\prime}\right)-f\left(s_{2}, s_{2}^{\prime}\right)\right| ; s_{1}, s_{2}, s_{1}^{\prime}, s_{2}^{\prime} \in[0, \zeta]:\left|s_{1}-s_{2}\right| \vee\left|s_{1}^{\prime}-s_{2}^{\prime}\right| \leq \eta\right\}
$$

that is the $\eta$-modulus of uniform continuity of $f$. The Arzelà-Ascoli Theorem asserts that the closure of $\mathcal{A} \subset \mathbf{C}\left([0, \zeta]^{2}, \mathbb{R}\right)$ is $\|\cdot\|$-compact if and only if $\sup _{f \in \mathcal{A}} \omega_{\eta}(f) \rightarrow 0$ as $\eta \rightarrow 0+$ and $\sup _{f \in \mathcal{A}}|f(0,0)|<\infty$. The desired result follows from the easy observation that $q_{\eta}(d) \leq \omega_{\eta}(d) \leq 2 q_{\eta}(d)$ (here, the second inequality is a consequence of the triangle inequality) and from the obvious fact that $d(0,0)=0$ for any $d \in \mathcal{D}$.

This lemma immediately implies the following tightness criterion.

Proposition 4.3. Let $\left(\mathbf{d}_{n}\right)_{n \in \mathbb{N}}$ be a sequence of $\mathbf{M}([0, \zeta])$-valued random variables. Their laws are tight on $\left(\mathbf{C}\left([0, \zeta]^{2}, \mathbb{R}\right),\|\cdot\|\right)$ if and only if $\lim _{\eta \rightarrow 0+} \sup _{n \in \mathbb{N}} \mathbf{P}\left(q_{\eta}\left(\mathbf{d}_{n}\right)>\varepsilon\right)=0$ for all $\varepsilon \in(0, \infty)$.

We now prove the following specific proposition that is used later in the proof of Theorem 1.2.

Proposition 4.4. Let $\mathbf{d}_{n}, \mathbf{d}_{n}^{*}, n \in \mathbb{N}$, be $\mathbf{M}([0, \zeta])$-valued r.v. such that 
(i) $\mathbf{d}_{n} \longrightarrow \mathbf{d}$ weakly on $\left(\mathbf{C}\left([0, \zeta]^{2}, \mathbb{R}\right),\|\cdot\|\right)$;

(ii) For all $n \in \mathbb{N}$ and for all $s, s^{\prime} \in[0, \zeta]$, a.s. $\mathbf{d}_{n}^{*}\left(s, s^{\prime}\right) \leq \mathbf{d}_{n}\left(s, s^{\prime}\right)$;

(iii) For all $s, s^{\prime} \in[0, \zeta],\left|\mathbf{d}_{n}^{*}\left(s, s^{\prime}\right)-\mathbf{d}_{n}\left(s, s^{\prime}\right)\right| \longrightarrow 0$ in probability.

Then, $\left(\mathbf{d}_{n}^{*}, \mathbf{d}_{n}\right) \longrightarrow(\mathbf{d}, \mathbf{d})$ weakly on $\left(\mathbf{C}\left([0, \zeta]^{2}, \mathbb{R}\right),\|\cdot\|\right)^{2}$.

Proof. By $(i)$ and Proposition 4.3, for all $\varepsilon \in(0, \infty), \lim _{\eta \rightarrow 0} \sup _{n \in \mathbb{N}} \mathbf{P}\left(q_{\eta}\left(\mathbf{d}_{n}\right)>\varepsilon\right)=0$. Since $\mathbf{d}_{n}$ and $\mathbf{d}_{n}^{*}$ are continuous, (ii) actually implies that a.s. for all $n \in \mathbb{N}$ and for all $s, s^{\prime} \in[0, \zeta], \mathbf{d}_{n}^{*}\left(s, s^{\prime}\right) \leq \mathbf{d}_{n}\left(s, s^{\prime}\right)$, which immediately entails $q_{\eta}\left(\mathbf{d}_{n}^{*}\right) \leq q_{\eta}\left(\mathbf{d}_{n}\right)$. Thus, $\lim _{\eta \rightarrow 0} \sup _{n \in \mathbb{N}} \mathbf{P}\left(q_{\eta}\left(\mathbf{d}_{n}^{*}\right)>\varepsilon\right)=0$, for all $\varepsilon \in(0, \infty)$. By Proposition 4.3, the laws of the r.v. $\left(\mathbf{d}_{n}^{*}, \mathbf{d}_{n}\right)$ are tight in $\mathbf{C}\left([0, \zeta]^{2}, \mathbb{R}\right)^{2}$ and we get the desired result because $(i)$ and (iii) easily entail the weak convergence of the finite dimensional marginals of $\left(\mathbf{d}_{n}^{*}, \mathbf{d}_{n}\right)$ to those of $(\mathbf{d}, \mathbf{d})$.

Induced metric spaces. Let $d \in \mathbf{M}([0, \zeta])$. We define the relation $\sim_{d}$ on $[0, \zeta]$ as follows: for all $s_{1}, s_{2} \in[0, \zeta], s_{1} \sim_{d} s_{2}$ if and only if $d\left(s_{1}, s_{2}\right)=0$. Clearly $\sim_{d}$ is an equivalence relation and we define the quotient space:

$$
E_{d}=[0, \zeta] / \sim_{d}, \quad \operatorname{proj}_{d}:[0, \zeta] \rightarrow E_{d}, \text { the canonical projection, } r_{d}=\operatorname{proj}_{d}(0) .
$$

We keep denoting $d$ the metric induced by $d$ on $E_{d}$. Since $d$ is continuous on $[0, \zeta]^{2}$, proj is continuous and $\left(E_{d}, d, r_{d}\right)$ is a pointed compact and connected metric space. We also equip $E_{d}$ with the pushforward measure $\mu_{d}$ of the Lebesgue measure on $[0, \zeta]$ via the canonical projection: namely, for all nonnegative measurable functions $f$ on $E_{d}$,

$$
\int_{E_{d}} f(x) \mu_{d}(d x)=\int_{0}^{\zeta} f\left(\operatorname{proj}_{d}(s)\right) d s .
$$

Note that $\mu_{d}$ is a finite measure with total mass $\zeta$.

Remark 4.5. Let $\left(E_{d}, d, r_{d}, \mu_{d}\right)$ be the compact metric space corresponding to the pseudometric $d \in \mathbf{M}([0, \zeta])$. Let $a, b \in(0, \infty)$. Set $d^{\prime}\left(s_{1}, s_{2}\right)=a d\left(s_{1} / b, s_{2} / b\right), s_{1}, s_{2} \in[0, b \zeta]$. Then, we easily check that $d^{\prime} \in \mathbf{M}([0, b \zeta])$ and $\left(E_{d^{\prime}}, d^{\prime}, r_{d^{\prime}}, \mu_{d^{\prime}}\right)$ is isometric to $\left(E_{d}, a d, r_{d}\right.$, $\left.b \mu_{d}\right)$.

Real trees. When $d \in \mathbf{M T}([0, \zeta])$, the resulting space $E_{d}$ is a real tree. More precisely, real trees are metric spaces that extend the definition of graph-trees; they are defined as follows.

Definition 4.6. let $(T, d)$ be a metric space; it is a real tree if and only if the following holds true.

(a) For any $\sigma_{1}, \sigma_{2} \in T$, there is a unique isometry $f:\left[0, d\left(\sigma_{1}, \sigma_{2}\right)\right] \rightarrow T$ such that $f(0)=\sigma_{1}$ and $f\left(d\left(\sigma_{1}, \sigma_{2}\right)\right)=\sigma_{2}$. Then, we set $\llbracket \sigma_{1}, \sigma_{2} \rrbracket:=f\left(\left[0, d\left(\sigma_{1}, \sigma_{2}\right)\right]\right)$.

(b) For any continuous injective function $g$ : $[0,1] \rightarrow T$, such that $g(0)=\sigma_{1}$ and $g(1)=\sigma_{2}$, $g([0,1])=\llbracket \sigma_{1}, \sigma_{2} \rrbracket$.

It turns out that the four points inequality is a metric characterisation of real trees. More precisely, if $(T, d)$ is a connected metric space, then $(T, d)$ is a real tree if and only if for any $\sigma_{1}, \sigma_{2}, \sigma_{3}, \sigma_{4} \in T$,

$$
d\left(\sigma_{1}, \sigma_{2}\right)+d\left(\sigma_{3}, \sigma_{4}\right) \leq\left(d\left(\sigma_{1}, \sigma_{3}\right)+d\left(\sigma_{2}, \sigma_{4}\right)\right) \vee\left(d\left(\sigma_{1}, \sigma_{4}\right)+d\left(\sigma_{2}, \sigma_{3}\right)\right) .
$$

We refer to Evans [15] or to Dress, Moulton and Terhalle [9] for a detailed account on this property.

Let us introduce some notation about real trees. Let $(T, d)$ be a compact pointed real tree. We distinguish a point $r \in T$ that is viewed as a root. We then define the length 
measure Length $(\cdot)$ on $T$ as the one-dimensional Hausdorff measure: namely, it is the unique Borel measure such that Length $\left(\llbracket \sigma, \sigma^{\prime} \rrbracket\right)=d\left(\sigma, \sigma^{\prime}\right)$, for all $\sigma, \sigma^{\prime} \in T$. Let us next introduce branch points: let $\sigma_{1}, \sigma_{2}, \sigma_{3} \in T$; then the geodesic paths $\llbracket \sigma_{1}, \sigma_{2} \rrbracket$, $\llbracket \sigma_{1}, \sigma_{3} \rrbracket$ and $\llbracket \sigma_{2}, \sigma_{3} \rrbracket$ have exactly one point in common that is called the branch point of $\sigma_{1}, \sigma_{2}, \sigma_{3}$ and that is denoted by $\operatorname{br}\left(\sigma_{1}, \sigma_{2}, \sigma_{3}\right)$; namely

$$
\left\{\operatorname{br}\left(\sigma_{1}, \sigma_{2}, \sigma_{3}\right)\right\}=\llbracket \sigma_{1}, \sigma_{2} \rrbracket \cap \llbracket \sigma_{1}, \sigma_{3} \rrbracket \cap \llbracket \sigma_{2}, \sigma_{3} \rrbracket .
$$

If we view $T$ as a family tree whose ancestor is $r$, then $\mathrm{br}\left(\sigma_{1}, \sigma_{2}, r\right)$ is the most recent common ancestor of $\sigma_{1}$ and $\sigma_{2}$ and we use the following notation

$$
\sigma_{1} \wedge \sigma_{2}=\operatorname{br}\left(\sigma_{1}, \sigma_{2}, r\right) .
$$

We next introduce the (extended) degree of any point $\sigma \in T$ as follows.

$\operatorname{deg}(\sigma)$ is the (possibly infinite) number of connected components of the open set $T \backslash\{\sigma\}$.

We say that $\sigma$ is a branch point if $\operatorname{deg}(\sigma) \geq 3$; we say that $\sigma$ is a leaf if $\operatorname{deg}(\sigma)=1$ and we say that $\sigma$ is simple if $\operatorname{deg}(\sigma)=2$. We shall use the following notation for the set of leaves of $T$

$$
\operatorname{Lf}(T):=\{\sigma \in T \backslash\{r\}: \operatorname{deg}(\sigma)=1\} .
$$

Definition 4.7. Let $\mu$ be a finite Borel measure on $T$; then $(T, d, r, \mu)$ is a continuum real tree in the sense of Aldous [3] if $T$ is compact and if

$$
\mu \text { is diffuse, the topological support of } \mu \text { is } T \text { and } \mu(T \backslash \operatorname{Lf}(T))=0 \text {. }
$$

Lemma 4.8. Let $\zeta \in(0, \infty)$ and $d \in \mathbf{M T}([0, \zeta])$. To simplify we denote by $(T, d, r, \mu)$ the pointed and measured compact real tree induced by the pseudo-metric $d$ and we denote by $p:[0, \zeta] \rightarrow T$ the canonical projection. We set

$$
U=\left\{s \in(0, \zeta): \forall s^{\prime} \in[0, \zeta] \backslash\{s\}, d(0, s)+d\left(s, s^{\prime}\right)>d\left(0, s^{\prime}\right)\right\} .
$$

We assume that $[0, \zeta] \backslash U$ is Lebesgue-negligible. Then, $T$ is a continuum real tree as defined in (4.6).

Proof. By construction and since $p$ is continuous, the topological support of $\mu$ is $T$. We next show that $p(U) \subset \operatorname{Lf}(T)$. Let $s \in(0, \zeta)$ be such that $p(s) \neq r$ and such that $\operatorname{deg}(p(s)) \geq 2$. Let $s^{\prime}$ be such that $p\left(s^{\prime}\right)$ belongs to a connected component of $T \backslash\{p(s)\}$ that does not contain $r$. Then, $p(s) \in \rrbracket r, p\left(s^{\prime}\right) \llbracket$, which implies that $d\left(p\left(s^{\prime}\right), r\right)=d(r, p(s))+d\left(p(s), p\left(s^{\prime}\right)\right)$, namely, $d\left(0, s^{\prime}\right)=d(0, s)+d\left(s, s^{\prime}\right)$. Thus, $s \notin U$. This proves that $p(U) \subset \operatorname{Lf}(T)$. Since $[0, \zeta] \backslash U$ is Lebesgue negligible, $T \backslash \operatorname{Lf}(T)$ is $\mu$-negligible.

It remains to prove that $\mu$ is diffuse. Let $s \in[0, \zeta]$. If $s \notin U$, then $p(s) \in T \backslash \operatorname{Lf}(T)$ that is $\mu$-negligible; thus $\mu(\{p(s)\})=0$. Assume now that $s \in U$ and suppose that $s^{\prime}$ is such that $d\left(s, s^{\prime}\right)=0$. The triangle inequality for $d$ implies that $d(0, s)=d\left(0, s^{\prime}\right)$. Since $s \in U$, we get $s=s^{\prime}$. Thus $p^{-1}(\{p(s)\})=\{s\}$ and $\mu(\{p(s)\})=0$, which completes the proof of the lemma.

Example 4.9. (Real trees spanned by graph-trees) Let $\mathrm{T}$ be a discrete graph-tree with a special vertex $\rho$ viewed as a root. We denote by $d_{\text {gr }}$ the graph-distance on $\mathrm{T}$. The real tree spanned by $\mathrm{T}$ is obtained by joining neighbouring vertices of $\mathrm{T}$ by a unit-length segment of the real line with its own metric. Formally, we can take $\widetilde{\mathrm{T}}=\{(\rho, 1)\} \cup \bigcup_{v \in \mathbb{T} \backslash\{\rho\}}\{v\} \times(0,1]$ and for all $\sigma=(v, s)$ and $\sigma^{\prime}=\left(v^{\prime}, s^{\prime}\right)$ in $\widetilde{\mathrm{T}}$, we set:

$$
\widetilde{d}_{\mathrm{gr}}^{\mathrm{T}}\left(\sigma, \sigma^{\prime}\right)= \begin{cases}d_{\mathrm{gr}}\left(v, v^{\prime}\right)+s+s^{\prime}-2 & \text { if } v \wedge v^{\prime} \notin\left\{v, v^{\prime}\right\}, \\ d_{\mathrm{gr}}\left(v, v^{\prime}\right)+s-s^{\prime} & \text { if } v^{\prime}=v \wedge v^{\prime} \text { and } v \neq v^{\prime}, \\ \left|s-s^{\prime}\right| & \text { if } v=v^{\prime}\end{cases}
$$


where $v \wedge v^{\prime}$ is the most recent common ancestor of $v$ and $v^{\prime}$ when we view $\mathrm{T}$ as a family tree whose ancestor is $\rho$. Clearly, $\left(\widetilde{\mathrm{T}}, \widetilde{d}_{\mathrm{gr}}^{\mathrm{T}}\right)$ is a real tree and if we identify $\mathrm{T}$ with $\mathrm{T} \times\{1\} \subset \widetilde{\mathrm{T}}$, we easily check that $\widetilde{d}_{\mathrm{gr}}^{\mathrm{T}}$ extends $d_{\mathrm{gr}}$. Note that $\left(\widetilde{\mathrm{T}}, \widetilde{d}_{\mathrm{gr}}^{\mathrm{T}}\right)$ is compact if and only if $\mathrm{T}$ is finite.

Example 4.10. (Real trees coded by continuous functions) Let $\zeta \in(0, \infty)$ and let $h$ : $[0, \zeta] \rightarrow \mathbb{R}$ be continuous. For all $s_{1}, s_{2} \in[0, \zeta]$, we set

$$
m_{h}\left(s_{1}, s_{2}\right)=\inf _{s \in\left[s_{1} \wedge s_{2}, s_{1} \vee s_{2}\right]} h\left(s_{2}\right) \text { and } d_{h}\left(s_{1}, s_{2}\right)=h\left(s_{1}\right)+h\left(s_{2}\right)-2 m_{h}\left(s_{1}, s_{2}\right) .
$$

We easily check that $d_{h} \in \mathbf{M T}([0, \zeta])$ and to simplify we denote by $\left(T_{h}, d_{h}, r_{h}, \mu_{h}\right)$ the induced metric space $\left(E_{d_{h}}, d_{h}, r_{d_{h}}, \mu_{d_{h}}\right)$ as defined in (4.2) and (4.3); we also denote by $p_{h}:[0, \zeta] \rightarrow T_{h}$ the canonical projection (instead of $\left.\operatorname{proj}_{d_{h}}\right) ;\left(T_{h}, d_{h}, r_{h}, \mu_{h}\right)$ is a pointed measured compact real tree that is the tree coded by the function $h$.

Let $h^{\prime}:[0, \zeta] \rightarrow \mathbb{R}$ be continuous. Then observe that

$$
\forall s_{1}, s_{2}, \in[0, \zeta], \quad\left|d_{h}\left(s_{1}, s_{2}\right)-d_{h^{\prime}}\left(s_{1}, s_{2}\right)\right| \leq 4 \sup _{s \in[0, \zeta]}\left|h(s)-h^{\prime}(s)\right|,
$$

which shows the continuity in $\mathbf{M T}([0, \zeta])$ of the application $h \mapsto d_{h}$.

Remark 4.11. Let $\left(T_{h}, d_{h}, r_{h}\right)$ be the real tree coded by the continuous function $h:[0, \zeta] \rightarrow$ $\mathbb{R}$ and the canonical projection $p_{h}$ as explained above. Suppose that $h$ is nonnegative and that $h(0)=0$. Then $d_{h}(0, s)=h(s)$ and for all $s_{1}, s_{2} \in[0, \zeta]$, we easily get $m_{h}\left(s_{1}, s_{2}\right)=$ $d_{h}\left(r_{h}, p_{h}\left(s_{1}\right) \wedge p_{h}\left(s_{2}\right)\right)$.

However, let $d \in \mathbf{M T}([0, \zeta])$ and set $h(s)=d(0, s), s \in[0, \zeta]$ that is a continuous function. The real tree $T=[0, \zeta] / \sim_{d}$ is in general different from the the real tree $T_{h}$ coded by $h$ : namely $d \neq d_{h}$. But let us mention that all compact real trees can be coded by (many) continuous functions (see D. [10] for more details on the coding of real trees).

Gromov-Hausdorff-Prokhorov metric. Let $\left(E_{1}, d_{1}, r_{1}, \mu_{1}\right)$ and $\left(E_{2}, d_{2}, r_{2}, \mu_{2}\right)$ be two pointed measured compact metric spaces: here $\mu_{1}$ and $\mu_{2}$ are finite measures on the respective Borel sigma-fields of $E_{1}$ and $E_{2}$, and $r_{1} \in E_{1}$ and $r_{2} \in E_{2}$ are distinguished points. The pointed Gromov-Hausdorff-Prokhorov distance (the GHP-distance for short) between $E_{1}$ and $E_{2}$ is defined by

$$
\begin{aligned}
\boldsymbol{\delta}_{\mathrm{GHP}}\left(E_{1}, E_{2}\right)=\inf \left\{d _ { E } ^ { \text { Haus } } \left(\phi_{1}\left(E_{1}\right)\right.\right. & \left., \phi_{2}\left(E_{2}\right)\right) \\
& \left.+d_{E}\left(\phi_{1}\left(r_{1}\right), \phi_{2}\left(r_{2}\right)\right)+d_{E}^{\text {Prok }}\left(\mu_{1} \circ \phi_{1}^{-1}, \mu_{2} \circ \phi_{2}^{-1}\right)\right\} .
\end{aligned}
$$

Here, the infimum is taken over all Polish spaces $\left(E, d_{E}\right)$ and all isometric embeddings $\phi_{i}: E_{i} \hookrightarrow E, i \in\{1,2\} ; d_{E}^{\text {Haus }}$ stands for the Hausdorff distance on the space of compact subsets of $E$ (namely, $d_{E}^{\text {Haus }}\left(K_{1}, K_{2}\right)=\inf \left\{\varepsilon \in(0, \infty): K_{1} \subset K_{2}^{(\varepsilon)}\right.$ and $\left.K_{2} \subset K_{1}^{(\varepsilon)}\right\}$, where $A^{(\varepsilon)}=\left\{y \in E: d_{E}(y, A) \leq \varepsilon\right\}$ for all non-empty $\left.A \subset E\right) ; d_{E}^{\text {Prok }}$ stands for the Prokhorov distance on the space of finite Borel measures on $E$ (namely, $d_{E}^{\text {Prok }}(\mu, \nu)=\inf \{\varepsilon \in(0, \infty)$ : $\mu(K) \leq \nu\left(K^{(\varepsilon)}\right)+\varepsilon$ and $\nu(K) \leq \mu\left(K^{(\varepsilon)}\right)+\varepsilon, \forall K \subset E$ compact $\}$ ); for all $i \in\{1,2\}, \mu_{i} \circ \phi_{i}^{-1}$ stands for the pushforward measure of $\mu_{i}$ via $\phi_{i}$.

Remark 4.12. Let $(E, d)$ be a Polish space and let $a, b, c \in(0, \infty)$. We denote by $d_{\text {Prok }}$ (resp. $\left.d_{\text {Prok }}^{a}\right)$ the Prokhorov distance on the space of finite Borel measures on $(E, d)$ (resp. $(E, a d)$ ) and we denote by $d_{\text {Haus }}$ (resp. $d_{\text {Haus }}^{a}$ ) the Hausdorff distance on the space of the compact subsets of $(E, d)$ (resp. $(E, a d)$ ). First note that $d_{\text {Haus }}^{a}=a d_{\text {Haus. }}$ Then, observe that $d_{\text {Prok }}(b \mu, c \mu)=|b-c| \mu(E)$ and that $(a \wedge b) d_{\text {Prok }}(\mu, \nu) \leq d_{\text {Prok }}^{a}(b \mu, b \nu) \leq(a \vee b) d_{\text {Prok }}(\mu, \nu)$.

Let $\left(E_{1}, d_{1}, r_{1}, \mu_{1}\right)$ and $\left(E_{2}, d_{2}, r_{2}, \mu_{2}\right)$ be two pointed measured compact metric spaces. We set $\left(E_{i}^{\prime}, d_{i}^{\prime}, r_{i}^{\prime}, \mu_{i}^{\prime}\right)=\left(E_{i}, a d_{i}, r_{i}, b \mu_{i}\right), i \in\{1,2\}$. Then, it is easy to check that $(a \wedge$ $b) \boldsymbol{\delta}_{\mathrm{GHP}}\left(E_{1}, E_{2}\right) \leq \boldsymbol{\delta}_{\mathrm{GHP}}\left(E_{1}^{\prime}, E_{2}^{\prime}\right) \leq(a \vee b) \boldsymbol{\delta}_{\mathrm{GHP}}\left(E_{1}, E_{2}\right)$. 
Example 4.13. Let $\mathrm{T}$ be a finite graph-tree that is equipped with its graph distance and with its counting measure: $\mathrm{m}=\sum_{v \in \mathrm{T}} \delta_{v}$. Let $\left(\widetilde{\mathrm{T}}, \widetilde{d}_{\mathrm{gr}}^{\mathrm{T}}\right)$ be the compact real tree spanned by $\mathrm{T}$ (see Example 4.9). We equip $\widetilde{\mathrm{T}}$ with its length measure Length. Up to obvious identifications, we can assume that $\mathrm{T} \subset \widetilde{\mathrm{T}}$. Then, we easily get $d_{\text {Haus }}(\mathrm{T}, \widetilde{\mathrm{T}}) \leq 1$, $d_{\text {Prok }}(\mathrm{m}$, Length $) \leq 2$ and thus, $\boldsymbol{\delta}_{\mathrm{GHP}}(\mathrm{T}, \widetilde{\mathrm{T}}) \leq 3$.

We next recall from Theorem 2.5 in Abraham, Delmas and Hoscheit [1] the following assertions: $\boldsymbol{\delta}_{\mathrm{GHP}}$ is a pseudo-metric (i.e. it is symmetric and it satisfies the triangle inequality) and $\boldsymbol{\delta}_{\mathrm{GHP}}\left(E_{1}, E_{2}\right)=0$ if and only if $E_{1}$ and $E_{2}$ are isometric, namely if and only if there exists a bijective isometry $\phi: E_{1} \rightarrow E_{2}$ such that $\phi\left(r_{1}\right)=r_{2}$ and such that $\mu_{2}=\mu_{1} \circ \phi^{-1}$. Denote by $\mathbb{M}$ the isometry classes of pointed measured compact metric spaces. Then, Theorem 2.5 in Abraham, Delmas and Hoscheit [1] asserts that

$$
\left(\mathrm{M}, \boldsymbol{\delta}_{\mathrm{GHP}}\right) \text { is a complete and separable metric space. }
$$

Proposition 4.14. Let $d, d^{\prime} \in \mathbf{M}([0, \zeta])$ and let $E_{d}$ and $E_{d^{\prime}}$ be the induced pointed measured compact metric spaces as defined by (4.2) and (4.3). Then

$$
\boldsymbol{\delta}_{\mathrm{GHP}}\left(E_{d}, E_{d^{\prime}}\right) \leq \frac{3}{2}\left\|d-d^{\prime}\right\| .
$$

Proof. We use the notation from (4.2) and we set $\mathcal{R}=\left\{\left(\operatorname{proj}_{d}(s), \operatorname{proj}_{d^{\prime}}(s)\right) ; s \in[0, \zeta]\right\}$ that is a relation on $E_{d} \times E_{d^{\prime}}$ since for all $x \in E_{d}$ and all $x^{\prime} \in E_{d^{\prime}}, \mathcal{R} \cap\left(\{x\} \times E_{d^{\prime}}\right)$ and $\mathcal{R} \cap\left(E_{d} \times\left\{x^{\prime}\right\}\right)$ are not empty. By the triangle inequality for pseudo-metrics, we get

$$
\operatorname{dis}(\mathcal{R}):=\sup \left\{\left|d(x, y)-d^{\prime}\left(x^{\prime}, y^{\prime}\right)\right| ;\left(x, x^{\prime}\right),\left(y, y^{\prime}\right) \in \mathcal{R}\right\} \leq\left\|d-d^{\prime}\right\| .
$$

We next set $G=E_{d} \sqcup E_{d^{\prime}}$ that is a disjoint union, and we define a metric $\delta$ on $G$ as follows: $\delta$ coincides with $d$ on $E_{d} \times E_{d}$ and with $d^{\prime}$ on $E_{d^{\prime}} \times E_{d^{\prime}}$ and for all $x \in E_{d}$ and all $x^{\prime} \in E_{d^{\prime}}$, we set

$$
\delta\left(x, x^{\prime}\right)=\inf \left\{d(x, y)+\frac{1}{2} \operatorname{dis}(\mathcal{R})+d^{\prime}\left(y^{\prime}, x^{\prime}\right) ;\left(y, y^{\prime}\right) \in \mathcal{R}\right\} .
$$

We easily check that $\delta$ is a separable compact metric on $G$. Thus, $(G, \delta)$ is Polish. The inclusions $E_{d} \hookrightarrow G$ and $E_{d^{\prime}} \hookrightarrow G$ are isometries and since $\left(r_{d}, r_{d^{\prime}}\right)=\left(\operatorname{proj}_{d}(0), \operatorname{proj}_{d^{\prime}}(0)\right) \in$ $\mathcal{R}$, we get

$$
\delta_{\text {Haus }}\left(E_{d}, E_{d^{\prime}}\right) \vee \delta\left(r_{d}, r_{d^{\prime}}\right) \leq \frac{1}{2} \operatorname{dis}(\mathcal{R}),
$$

where $\delta_{\text {Haus }}$ stands for the Hausdorff distance of the space of compacts subsets of $G$.

Let $K$ be a closed (and thus compact) subset of $G$. We set $Q=E_{d} \cap K$ and $Q^{\prime}=E_{d^{\prime}} \cap K$ that are also compact subsets of $G$. Note that $\mu_{d}(K)=\mu_{d}(Q)$ and $\mu_{d^{\prime}}(K)=\mu_{d^{\prime}}\left(Q^{\prime}\right)$. We set $C=\operatorname{proj}_{d}^{-1}(Q), Q_{0}^{\prime}=\operatorname{proj}_{d^{\prime}}(C)$ and $C^{\prime}=\operatorname{proj}_{d^{\prime}}^{-1}\left(Q_{0}^{\prime}\right)$. Since $\operatorname{proj}_{d}$ and $\operatorname{proj}_{d^{\prime}}$ are continuous, $C$ and $C^{\prime}$ are compact subsets of $[0, \zeta]$ and $Q_{0}^{\prime}$ is a compact subset of $E_{d^{\prime}}$. Denote by $\ell$ the Lebesgue measure on $[0, \infty)$. Observe that $C \subset C^{\prime}$, which implies that $\mu_{d}(K)=\mu_{d}(Q)=\ell(C) \leq \ell\left(C^{\prime}\right)=\mu_{d^{\prime}}\left(Q_{0}^{\prime}\right)$. Let $x^{\prime} \in Q_{0}^{\prime}$. There exists $s \in C$ such that $\operatorname{proj}_{d^{\prime}}(s)=x^{\prime}$. But note that $x:=\operatorname{proj}_{d}(s) \in Q$ and thus $\left(x, x^{\prime}\right) \in \mathcal{R}$. Set $\eta:=\frac{1}{2} \operatorname{dis}(\mathcal{R})$; then we have proved that $\mu_{d}(K) \leq \mu_{d^{\prime}}\left(K^{\eta}\right)$ where $K^{\eta}=\{z \in G: \delta(z, K) \leq \eta\}$. Similarly, we prove that $\mu_{d^{\prime}}(K) \leq \mu_{d}\left(K^{\eta}\right)$ which implies that $\delta_{\text {Prok }}\left(\mu_{d}, \mu_{d^{\prime}}\right) \leq \eta$, where $\delta_{\text {Prok }}$ stands for the Prohorov distance on the space of finite Borel measures on $G$. This inequality combined with (4.14) implies that $\delta_{\mathrm{GHP}}\left(E_{d}, E_{d^{\prime}}\right) \leq \frac{3}{2} \operatorname{dis}(\mathcal{R})$, which entails (4.12) by (4.13).

\subsection{Scaling limit of the range of biased RWs on trees}

Let $T$ be an infinite rooted ordered tree. We fix $\lambda \in(1, \infty)$ and we denote by $\left(Y_{n}\right)_{n \in \mathbb{N}}$ the $\lambda$-biased RW on $T$ whose transition probabilities are given by (3.36). We make $T$ a real tree by joining neighbouring vertices by a line isometric to $[0,1]$ as explained in 
Example 4.9 and we keep denoting by $\left(T, d_{\mathrm{gr}}, \varnothing\right)$ the resulting rooted real tree. We also denote simply by $\left(Y_{s}\right)_{s \in[0, \infty)}$ the continuous interpolation of $Y$ : namely, for all $n \in \mathbb{N}$ and all $s \in[n, n+1], Y_{s}$ is the unique point of the line $\llbracket Y_{n}, Y_{n+1} \rrbracket$ in the (spanned) real tree $T$ such that $d_{\mathrm{gr}}\left(Y_{n}, Y_{s}\right)=s-n$.

Let $\zeta \in(0, \infty)$. For all $n \in \mathbb{N}$, we then set

$$
\mathcal{R}_{n}=\left\{Y_{s} ; s \in[0, \zeta n]\right\}
$$

that is a random compact real tree. We equip $\mathcal{R}_{n}$ with the occupation measure $m_{\text {occ }}^{(n)}$ induced by the RW, namely $\int_{\mathcal{R}_{n}} f(\sigma) m_{\text {occ }}^{(n)}(d \sigma)=\int_{0}^{\zeta} f\left(Y_{s n}\right) d s$.

Proposition 4.15. We keep the above notation. Let $\left(a_{n}\right)_{n \in \mathbb{N}}$ be a sequence of positive real numbers. We assume that $\left(Y_{n}\right)_{n \in \mathbb{N}}$ is recurrent, that $\lim _{n \rightarrow \infty} a_{n}^{-1} \log n=0$ and that there exists a continuous random process $\left(H_{s}\right)_{s \in[0, \zeta]}$ such that

$$
\left(\frac{1}{a_{n}} d_{\mathrm{gr}}\left(\varnothing, Y_{n s}\right)\right)_{s \in[0, \zeta]} \underset{n \rightarrow \infty}{\stackrel{\text { (law) }}{\longrightarrow}}\left(H_{s}\right)_{s \in[0, \zeta]}
$$

weakly on $\mathbf{C}([0, \zeta], \mathbb{R})$. Then, jointly with (4.15), the following convergence

$$
\left(\mathcal{R}_{n}, \frac{1}{a_{n}} d_{\mathrm{gr}}, \varnothing, m_{\mathrm{occ}}^{(n)}\right) \underset{n \rightarrow \infty}{\stackrel{\text { (law) }}{\longrightarrow}}\left(T_{H}, d_{H}, r_{H}, \mu_{H}\right)
$$

holds weakly on the space of rooted measured compact metric spaces IM equipped with the Gromov-Hausdorff-Prokhorov distance. Here, $\left(T_{H}, d_{H}, r_{H}, \mu_{H}\right)$ stands for the real tree coded by $H$ as in Example 4.10.

Proof. For all $s \in[0, \zeta]$, we set $H_{s}^{(n)}=\frac{1}{a_{n}} d_{\mathrm{gr}}\left(\varnothing, Y_{n s}\right)$ and for all $s, s^{\prime} \in[0, \zeta], d_{n}\left(s, s^{\prime}\right)=$ $H_{s}^{(n)}+H_{s^{\prime}}^{(n)}-2 \min _{r \in\left[s \wedge s^{\prime}, s \vee s^{\prime}\right]} H_{r}^{(n)}$, that is the tree pseudo-distance coded by $H^{(n)}$ as in Example 4.10. Clearly, by (4.9), (4.15) implies that $d_{n} \rightarrow d_{H}$ weakly on $\mathbf{C}\left([0, \zeta]^{2}, \mathbb{R}\right)$. Then we set $d_{n}^{*}\left(s, s^{\prime}\right)=a_{n}^{-1} d_{\mathrm{gr}}\left(Y_{n s}, Y_{n s^{\prime}}\right)$. Thus $d_{n}^{*}\left(s, s^{\prime}\right) \leq d_{n}\left(s, s^{\prime}\right)$ and by (3.37) in Lemma 3.17, we get for all $\varepsilon>0$ that

$$
\begin{aligned}
\mathbf{P}\left(d_{n}\left(s, s^{\prime}\right)-d_{n}^{*}\left(s, s^{\prime}\right)>\varepsilon\right) & \leq \mathbf{P}\left(\left|Y_{\lfloor n s\rfloor}\right|+\left|Y_{\left\lfloor n s^{\prime}\right\rfloor}\right|-2 \min _{\lfloor n s\rfloor \leq k \leq\left\lfloor n s^{\prime}\right\rfloor}\left|Y_{k}\right|\right. \\
& \leq\left(\left\lfloor n s^{\prime}\right\rfloor-\lfloor n s\rfloor\right) \frac{\lambda-1}{\lambda^{\frac{1}{2} \varepsilon a_{n}-1}-1} \underset{n \rightarrow \infty}{\longrightarrow} 0 .
\end{aligned}
$$

By Proposition 4.4, $\left(d_{n}^{*}, d_{n}\right) \rightarrow\left(d_{H}, d_{H}\right)$ weakly on $\mathbf{C}\left([0, \zeta]^{2}, \mathbb{R}\right)^{2}$. This implies the desired result by Proposition 4.14 and because the compact measured rooted tree induced by the pseudo-metric $d_{n}^{*}$ is isometric to $\left(\mathcal{R}_{n}, \frac{1}{a_{n}} d_{\mathrm{gr}}, \varnothing, m_{\mathrm{occ}}^{(n)}\right)$.

We then derive from the previous proposition (the easy part) and from a result due to A. Dembo and N. Sun [8] (the difficult part) that the scaling limit of the range of critical biased RWs on a supercritical multi-type GW-tree is the Brownian tree. More precisely, we consider a $N$-type GW-tree; for all $k \in\{1, \ldots, N\}$ we denote by $\boldsymbol{\mu}(k, \cdot)$ a probability measure on $\mathbb{W}_{N}=\bigcup_{n \in \mathbb{N}}\{1, \ldots, N\}^{n}$, the set of finite words written in the alphabet $\{1, \ldots, N\}$. For all $p \in(0, \infty)$, the $p$-th moment matrix $M_{p}=\left(m_{p}(k, \ell)\right)_{1 \leq k, \ell \leq N}$ is given by

$$
m_{p}(k, \ell)=\sum_{n \in \mathbb{N}} \sum_{1 \leq w_{1}, \ldots, w_{n} \leq N} \boldsymbol{\mu}\left(k,\left(w_{1}, \ldots, w_{n}\right)\right)\left(\#\left\{j \in\{1, \ldots, n\}: w_{j}=\ell\right\}\right)^{p} .
$$

We assume the following.

(a) There exists $p \in(4, \infty)$ such that for all $k, \ell \in\{1, \ldots, N\}, m_{p}(k, \ell)<\infty$. 
(b) There exists an integer $n_{0} \geq 1$, such that $M_{1}^{n_{0}}$ has only strictly positive entries.

(c) Let $\lambda$ be the Perron-Frobenius eigenvalue of $M_{1}$. We assume that $\lambda>1$.

Let $\mathbf{T}=\left(T, \varnothing,\left(a_{x}\right)_{x \in T}\right)$ be a $N$-type $\mathrm{GW}(\boldsymbol{\mu})$-tree. Namely, $T$ is a random rooted ordered tree, $a_{x} \in\{1, \ldots, N\}$ is the type of vertex $x \in T$ and $\mathbf{T}$ satisfies the following property: recall that $k_{\varnothing}(T)$ is the number of children of the root and recall that for all $j \in\left\{1, \ldots, k_{\varnothing}(T)\right\}, \theta_{(j)} \mathbf{T}$ stands for the tree stemming form the $j$-th child of $\varnothing$ (equipped with the types of corresponding vertices). Then, conditionally given $a_{\varnothing}$, the types $\left(a_{(1)}, \ldots, a_{\left(k_{\varnothing}(T)\right)}\right)$ of the children of the root have conditional law $\boldsymbol{\mu}\left(a_{\varnothing}, \cdot\right)$ and conditionally given $\left(a_{(1)}, \ldots, a_{\left(k_{\varnothing}(T)\right)}\right)$, the subtrees $\theta_{(1)} \mathbf{T}, \ldots, \theta_{\left(k_{\varnothing}(T)\right)} \mathbf{T}$ are independent $\mathrm{GW}(\boldsymbol{\mu})$-trees.

Then, conditionally given $T$, let $\left(Y_{n}\right)_{n \in \mathbb{N}}$ be the $\lambda$-biased RW on $T$ started at $\varnothing$ and denote by $\left(Y_{s}\right)_{s \in[0, \infty)}$ its continuous interpolation as explained above. Under Assumptions $(a),(b)$ and $(c)$ on $\boldsymbol{\mu}$, Theorem 1.1 in A. Dembo and N. Sun [8] p. 3 asserts that there exists $\sigma \in(0, \infty)$, a constant that depends only on $\mu$, such that a.s. on the non-extinction event $\{|T|=\infty\}$,

$$
\left(\frac{1}{\sigma \sqrt{n}} d_{\mathrm{gr}}\left(\varnothing, Y_{n s}\right)\right)_{s \in[0, \infty)} \underset{n \rightarrow \infty}{\stackrel{\text { (law) }}{\longrightarrow}}\left(\left|B_{s}\right|\right)_{s \in[0, \infty)}
$$

where $\left(B_{s}\right)_{s \in[0, \infty)}$ is a standard linear Brownian motion such that $B_{0}=0$ a.s. By Proposition 4.15 , we immediately get the following new result.

Corollary 4.16. We keep the notation as above. Let $\zeta \in(0, \infty)$. Let $\mathcal{R}_{n}=\left\{Y_{s} ; s \in[0, n \zeta]\right\}$ be the range of $Y$ up to time $n \zeta$ and let $m_{\text {occ }}^{(n)}$ be the occupation measure of $Y$ on $\mathcal{R}_{n}$ as introduced before. Under Assumptions (a), (b) and (c) on $\boldsymbol{\mu}$, a.s. on the event $\{|T|=\infty\}$, we get

$$
\left(\mathcal{R}_{n}, \frac{1}{\sigma \sqrt{n}} d_{\text {gr }}, \varnothing, m_{\text {occ }}^{(n)}\right) \underset{n \rightarrow \infty}{\stackrel{\text { (law) }}{\longrightarrow}}\left(T_{|B|}, d_{|B|}, r_{|B|}, \mu_{|B|}\right)
$$

weakly on the space of rooted measured compact metric spaces IM equipped with the Gromov-Hausdorff-Prokhorov distance. Here, $\left(T_{|B|}, d_{|B|}, r_{|B|}, \mu_{|B|}\right)$ stands for the real tree coded by the reflected Brownian motion $\left(\left|B_{s}\right|\right)_{s \in[0, \zeta]}$.

In literature, the first scaling limit for the range of tree-valued RWs appears in D. [12]: in this paper the tree is b-ary and the RW is slightly super-critical (see Theorem 2.1 [12] p. 2224; Lemma 3.7 [12] p. 2241 also contains a local law of large numbers for the range). When $T$ is a supercritical single-type GW-tree, Y. Peres and O. Zeitouni [30] have first proved (4.16) when the offspring distribution has exponential moments (see Theorem 1 [30], p. 596). Then, E. Aïdékon and L. de Raphélis in [2] have proved (4.16) for supercritical single-type GW-tree under a second moment assumption and they also proved (4.17) in these cases (Theorem 1.1 [2], p. 645). In the same article, they extend (4.16) and (4.17) to RWs in random environment on GW-trees (see Theorem 6.1 [2], p. 660).

\subsection{Snake metrics}

Snakes are path-valued processes that provide a nice parametrization of the spatial positions of a population whose genealogy is a continuum tree and that are scaling limits of branching random walks. Snake processes, and in particular the Brownian snake, has been introduced by J-F. Le Gall in [21] to study fine properties of super-Brownian motion. In this section, we first recall basic definitions on snakes, in a deterministic setting and in dimension 1 . Then, we introduce a pseudo-metric derived from a snake, we study its continuity properties and we show that snake metrics actually yield real-trees. Finally, we prove elementary geometric properties of such real trees that are deterministic version of the cactus introduced by Curien, Le Gall and Miermont in [7]. 
Definition 4.17. We fix $\zeta \in(0, \infty)$ and we denote by $\mathbf{C}([0, \zeta], \mathbb{R})$ the space of the continuous functions from $[0, \zeta]$ to $\mathbb{R}$; it is a Banach space when equipped with the uniform norm $\|\cdot\|_{\infty}$. We also denote by $\mathbf{C}_{0}$ the space of continuous functions from $[0, \infty)$ to $\mathbb{R}$ that is a Polish space when equipped with the following metric:

$$
\forall w, w^{\prime} \in \mathbf{C}_{0}, \quad \delta_{\mathbf{u}}\left(w, w^{\prime}\right)=\sum_{n \in \mathbb{N}} 2^{-n-1} \min \left(1, \sup _{r \in[0, n]}\left|w(r)-w^{\prime}(r)\right|\right) .
$$

(a) We denote by $\mathbf{C}\left([0, \zeta], \mathbf{C}_{0}\right)$ the space of the $\delta_{\mathrm{u}}$-continuous functions from $[0, \zeta]$ to $\mathbf{C}_{0}$ equipped with the distance $\Delta^{*}\left(w, w^{\prime}\right)=\sup _{s \in[0, \zeta]} \delta_{\mathrm{u}}\left(w_{s}(\cdot), w_{s}^{\prime}(\cdot)\right)$, for all $w, w^{\prime} \in$ $\mathbf{C}\left([0, \zeta], \mathbf{C}_{0}\right)$. We next equip the product space $\mathbf{C}([0, \zeta], \mathbb{R}) \times \mathbf{C}\left([0, \zeta], \mathbf{C}_{0}\right)$ with the following distance: for all $(h, w),\left(h^{\prime}, w^{\prime}\right) \in \mathbf{C}([0, \zeta], \mathbb{R}) \times \mathbf{C}\left([0, \zeta], \mathbf{C}_{0}\right)$,

$$
\Delta\left((h, w),\left(h^{\prime}, w^{\prime}\right)\right)=\left\|h-h^{\prime}\right\|_{\infty}+\Delta^{*}\left(w, w^{\prime}\right) .
$$

(b) We denote by $\boldsymbol{\Sigma}([0, \zeta])$ the space of the $\mathbb{R}$-valued snakes; namely, the space of $(h, w) \in \mathbf{C}([0, \zeta], \mathbb{R}) \times \mathbf{C}\left([0, \zeta], \mathbf{C}_{0}\right)$ that satisfy $h \geq 0, h(0)=h(\zeta)=0$ and the following.

(b1) For all $s \in[0, \zeta]$ and for all $r \in[h(s), \infty), w_{s}(r)=w_{s}(h(s))=: \widehat{w}_{s}$.

(b2) For all $s_{1}, s_{2} \in[0, \zeta]$ and for all $r \in\left[0, m_{h}\left(s_{1}, s_{2}\right)\right], w_{s_{1}}(r)=w_{s_{2}}(r)$, where we recall from (4.8) the definition of $m_{h}\left(s_{1}, s_{2}\right)$.

We refer to $(b 2)$ as to the snake property. The function $h$ is called the lifetime process and the function $\left(\widehat{w}_{s}\right)_{s \in[0, \zeta]}$ is called the endpoint process of the snake.

We easily check that $\left(\mathbf{C}\left([0, \zeta], \mathbf{C}_{0}\right), \Delta^{*}\right)$ and $\left(\mathbf{C}([0, \zeta], \mathbb{R}) \times \mathbf{C}\left([0, \zeta], \mathbf{C}_{0}\right), \Delta\right)$ are Polish spaces and that $\boldsymbol{\Sigma}([0, \zeta])$ is a $\Delta$-closed subset.

The following lemma is used in the proof of Theorem 1.2.

Lemma 4.18. Let $(h, w) \in \boldsymbol{\Sigma}([0, \zeta])$. For all $\eta \in(0, \infty)$, we set $\omega_{\eta}(w)=\sup \left\{\delta_{\mathrm{u}}\left(w_{s}, w_{s^{\prime}}\right) ; s, s^{\prime}\right.$ $\left.\in[0, \zeta]:\left|s-s^{\prime}\right| \leq \eta\right\}$ and $\omega_{\eta}(\widehat{w})=\sup \left\{\left|\widehat{w}_{s}-\widehat{w}_{s^{\prime}}\right| ; s, s^{\prime} \in[0, \zeta]:\left|s-s^{\prime}\right| \leq \eta\right\}$. Then, $\omega_{\eta}(w) \leq 2 \omega_{\eta}(\widehat{w})$.

Proof. For all $s \in[0, \zeta]$ and for all $r \in[0, h(s)]$ set $\alpha_{s, r}=\sup \left\{s^{\prime} \in[0, s]: h\left(s^{\prime}\right) \leq r\right\}$ and $\beta_{s, r}=\inf \left\{s^{\prime} \in[s, \zeta]: h\left(s^{\prime}\right) \leq r\right\}$. Then, Definition $4.17(b 2)$ implies that $w_{s}(r)=w_{s^{\prime}}(r)$ for all $s^{\prime} \in\left[\alpha_{s, r}, \beta_{s, r}\right]$ and in particular, $\widehat{w}_{\alpha_{s, r}}=\widehat{w}_{\beta_{s, r}}=w_{s}(r)$ since $r=h\left(\alpha_{s, r}\right)=h\left(\beta_{s, r}\right)$.

Next, fix $s, s^{\prime} \in[0, \zeta]$ such that $\left|s-s^{\prime}\right| \leq \eta$. To simplify we set $m=m_{h}\left(s, s^{\prime}\right)$. Let $r_{*} \in$ $[m, h(s)]$ be such that $\left|w_{s}\left(r_{*}\right)-w_{s}(m)\right|=\max _{r \in[m, h(s)]}\left|w_{s}(r)-w_{s}(m)\right|$. Suppose that $s \leq s^{\prime}$ (resp. that $\left.s^{\prime} \leq s\right)$, then $\beta_{s, r_{*}}, \beta_{s, m} \in\left[s, s^{\prime}\right]\left(\operatorname{resp} . \alpha_{s, r_{*}}, \alpha_{s, m} \in\left[s^{\prime}, s\right]\right)$ and $\left|w_{s}\left(r_{*}\right)-w_{s}(m)\right|=$ $\left|\widehat{w}_{\beta_{s, r_{*}}}-\widehat{w}_{\beta_{s, m}}\right|$ (resp. $\left.=\left|\widehat{w}_{\alpha_{s, r_{*}}}-\widehat{w}_{\alpha_{s, m}}\right|\right)$. Thus, $\max _{r \in[m, h(s)]}\left|w_{s}(r)-w_{s}(m)\right| \leq \omega_{\eta}(\widehat{w})$. Similarly, $\max _{r \in\left[m, h\left(s^{\prime}\right)\right]}\left|w_{s^{\prime}}(r)-w_{s^{\prime}}(m)\right| \leq \omega_{\eta}(\widehat{w})$. By Definition $4.17(b 2)$, we get $w_{s}(m)=$ $w_{s^{\prime}}(m)$ and $\sup _{r \in[0, \infty)}\left|w_{s}(r)-w_{s^{\prime}}(r)\right| \leq \max _{r \in[m, h(s)]}\left|w_{s}(r)-w_{s}(m)\right|+\max _{r \in\left[m, h\left(s^{\prime}\right)\right]} \mid w_{\mathcal{s}^{\prime}}(r)-$ $w_{s^{\prime}}(m) \mid$, which easily implies the desired result.

Definition 4.19. Let $\zeta \in(0, \infty)$ and let $(h, w) \in \boldsymbol{\Sigma}([0, \zeta])$. Recall from (4.8) the definition of $m_{h}(\cdot, \cdot)$ and recall from Definition $4.17(b 1)$ the definition of $\widehat{w}$. For all $s_{1}, s_{2} \in[0, \zeta]$, we set

$$
\begin{gathered}
M_{h, w}\left(s_{1}, s_{2}\right)=\min \left(\min \left\{w_{s_{1}}(r) ; r \in\left[m_{h}\left(s_{1}, s_{2}\right), h\left(s_{1}\right)\right]\right\}, \min \left\{w_{s_{2}}(r) ; r \in\left[m_{h}\left(s_{1}, s_{2}\right), h\left(s_{2}\right)\right]\right\}\right) \\
\quad \text { and } d_{h, w}\left(s_{1}, s_{2}\right)=\widehat{w}_{s_{1}}+\widehat{w}_{s_{2}}-2 M_{h, w}\left(s_{1}, s_{2}\right) .
\end{gathered}
$$

We call $d_{h, w}$ the snake metric associated with $(h, w)$ (see Lemma 4.22 below).

Lemma 4.20. Let $\zeta \in[0, \infty)$ and for all $n \in \mathbb{N}$, let $\left(h_{n}, w^{(n)}\right) \in \mathbf{\Sigma}([0, \zeta])$ that converges to $(h, w)$ in $\mathbf{C}([0, \zeta], \mathbb{R}) \times \mathbf{C}\left([0, \zeta], \mathbf{C}_{0}\right)$ equipped with $\Delta$ as in Definition $4.17(a)$. Recall that $\|\cdot\|$ stands for the uniform norm on $\mathbf{C}\left([0, \zeta]^{2}, \mathbb{R}\right)$. Then, $\lim _{n \rightarrow \infty}\left\|d_{h_{n}}-d_{h}\right\|=\lim _{n \rightarrow \infty} \| d_{h_{n}, w^{(n)}}-$ $d_{h, w} \|=0$ (see (4.8) and (4.19)). 
Proof. The first limit follows from (4.9). To prove the second one, we fix $\varepsilon \in(0,1)$ and we set $a=1+\sup _{n \in \mathbb{N}} \max _{s \in[0, \zeta]}\left|h_{n}(s)\right|$. Let $n_{1} \in \mathbb{N}$ such that $\Delta\left(\left(h_{n}, w^{(n)}\right),(h, w)\right) \leq 2^{-a-2} \varepsilon$, for all $n \geq n_{1}$. Thus, for all $n \geq n_{1}$ and for all $s \in[0, \zeta]$,

$$
\left\|w_{s}^{(n)}-w_{s}\right\|_{\infty}:=\sup _{r \in[0, \infty)}\left|w_{s}^{(n)}(r)-w_{s}(r)\right|=\sup _{r \in[0, a]}\left|w_{s}^{(n)}(r)-w_{s}(r)\right| \leq \varepsilon .
$$

Fix $s_{1}, s_{2} \in[0, \zeta]$ and set $m_{n}=m_{h_{n}}\left(s_{1}, s_{2}\right)$ and $m=m_{h}\left(s_{1}, s_{2}\right)$. By (4.20), we get

$$
\forall n \geq n_{1}, \forall j \in\{1,2\}, \quad\left|\min _{r \in\left[m_{n}, h_{n}\left(s_{j}\right)\right]} w^{(n)}(r)-\min _{r \in\left[m_{n}, h_{n}\left(s_{j}\right)\right]} w_{s_{j}}(r)\right| \leq\left\|w_{s_{j}}^{(n)}-w_{s_{j}}\right\|_{\infty} \leq \varepsilon .
$$

Next, for all uniformly continuous $g:[0, \infty) \rightarrow \mathbb{R}$, and for all $\eta \in(0, \infty)$, we use the notation $\omega(g, \eta)=\sup \left\{\left|g(s)-g\left(s^{\prime}\right)\right| ; s, s^{\prime} \in[0, \infty):\left|s-s^{\prime}\right| \leq \eta\right\}$ for the $\eta$-uniform modulus of continuity of $g$. We recall that $\left\|h-h_{n}\right\|_{\infty} \leq \Delta\left(\left(h_{n}, w^{(n)}\right),(h, w)\right)$. Observe that for all $j \in\{1,2\}$ and all $n \in \mathbb{N}$,

$$
\left|\min _{r \in\left[m_{n}, h_{n}\left(s_{j}\right)\right]} w_{s_{j}}(r)-\min _{r \in\left[m, h\left(s_{j}\right)\right]} w_{\left.s_{j}\right)}(r)\right| \leq \omega\left(w_{s_{j}},\left\|h-h_{n}\right\|_{\infty}\right) .
$$

By the definition (4.19) of $d_{h_{n}, w^{(n)}}$, by (4.20), (4.21) and (4.22), for all $n \geq n_{1}$, we get the following:

$$
\left|d_{h_{n}, w^{(n)}}\left(s_{1}, s_{2}\right)-d_{h, w}\left(s_{1}, s_{2}\right)\right| \leq 4 \varepsilon+3 \omega\left(w_{s_{1}},\left\|h-h_{n}\right\|_{\infty}\right)+3 \omega\left(w_{s_{2}},\left\|h-h_{n}\right\|_{\infty}\right) .
$$

Since $w$ is uniformly $\delta_{\mathrm{u}}$-continuous on $[0, \zeta]$, there exist $\sigma_{1}, \ldots, \sigma_{p} \in[0, \zeta]$ such that for all $s \in[0, \zeta]$, there exists $k \in\{1, \ldots, p\}$, such that $\left\|w_{s}-w_{\sigma_{k}}\right\|_{\infty} \leq \varepsilon$, which implies that $\left|\omega\left(w_{s}, \eta\right)-\omega\left(w_{\sigma_{k}}, \eta\right)\right| \leq 2 \varepsilon$, for all $\eta \in(0, \infty)$. By (4.23), it implies that

$$
\left\|d_{h_{n}, w^{(n)}}-d_{h, w}\right\| \leq 16 \varepsilon+6 \max _{1 \leq k \leq p} \omega\left(w_{\sigma_{k}},\left\|h-h_{n}\right\|_{\infty}\right),
$$

which implies $\lim \sup _{n \rightarrow \infty}\left\|d_{h_{n}, w^{(n)}}-d_{h, w}\right\| \leq 16 \varepsilon$, for all $\varepsilon \in(0, \infty)$. It completes the proof of the lemma.

Remark 4.21. Let $(h, w) \in \boldsymbol{\Sigma}([0, \zeta])$. Definition $4.17(b)$ means that $w$ is actually defined on the real tree $\left(T_{h}, d_{h}, r_{h}, \mu_{h}\right)$ coded by $h$ (as defined in Remark 4.10). Indeed, let $s_{1}, s_{2} \in[0, \zeta]$ be such that $d_{h}\left(s_{1}, s_{2}\right)=0$; then $h\left(s_{1}\right)=h\left(s_{2}\right)=m_{h}\left(s_{1}, s_{2}\right)$ and Definition 4.17 (b) implies that $w_{s_{1}}=w_{s_{2}}$. Up to a slight abuse of notation, it therefore makes sense to define $w$ on $T_{h}$ as follows: for all $\sigma \in T_{h}$ and for all $s \in[0, \zeta]$ such that $\sigma=p_{h}(s)$, then

$$
w_{\sigma}(\cdot):=w_{s}(\cdot) \text { and } \widehat{w}_{\sigma}:=\widehat{w}_{s},
$$

where we recall that $p_{h}:[0, \zeta] \rightarrow T_{h}$ stands for the canonical projection. It is easy to check that $w: T_{h} \rightarrow \mathbf{C}_{0}$ is continuous. Moreover, Definition $4.17(b)$ combined with the argument of the proof of Lemma 4.18 entails the following.

$$
\forall \sigma \in T_{h}, \forall \gamma \in \llbracket r_{h}, \sigma \rrbracket, \quad w_{\sigma}\left(d_{h}\left(r_{h}, \gamma\right)\right)=\widehat{w}_{\gamma} .
$$

We also get the following: let $\sigma_{1}, \sigma_{2} \in T_{h}$ and $s_{1}, s_{2} \in[0, \zeta]$ such that $p_{h}\left(s_{i}\right)=\sigma_{i}, i \in\{1,2\}$; then

$$
M_{h, w}\left(s_{1}, s_{2}\right)=\min _{\gamma \in \llbracket \sigma_{1}, \sigma_{2} \rrbracket} \widehat{w}_{\gamma} \text { and thus } d_{h, w}\left(s_{1}, s_{2}\right)=\widehat{w}_{\sigma_{1}}+\widehat{w}_{\sigma_{2}}-2 \min _{\gamma \in \llbracket \sigma_{1}, \sigma_{2} \rrbracket} \widehat{w}_{\gamma} .
$$

Up to a slight abuse of notation, it makes sense to view $M_{h, w}$ and $d_{h, w}$ as continuous functions from $T_{h} \times T_{h}$ to $\mathbb{R}$. 
Lemma 4.22. Let $\zeta \in(0, \infty)$ and let $(h, w) \in \mathbf{\Sigma}([0, \zeta])$. Let $d_{h, w}$ be the associated snake metric as in Definition 4.19. Then, $d_{h, w} \in \mathbf{M T}([0, \zeta])$. Namely, it is a continuous pseudometric on $[0, \zeta]$ that satisfies the four points inequality (4.1).

Proof. We first prove the continuity of $d_{h, w}$. To that end, for all $a, b \in[0, \infty)$ and all $s \in[0, \zeta]$ we set $\phi_{a, b}(s)=\min \left\{w_{s}(r) ; r \in[a \wedge b, a \vee b]\right\}$. First note that $\left|\phi_{a, b}(s)-\phi_{a, b}\left(s^{\prime}\right)\right| \leq$ $\sup _{r \in[0, a \vee b]}\left|w_{s}(r)-w_{s^{\prime}}(r)\right|$. Then, observe that for a fixed $s,(a, b) \mapsto \phi_{a, b}(s)$ is continuous. This easily implies that $(a, b, s) \mapsto \phi_{a, b}(s)$ is continuous. Therefore, $d_{h, w}$ is continuous on $[0, \zeta]^{2}$.

Let $s_{1}, s_{2}, s_{3}, s_{4} \in[0, \zeta]$. We set $X_{1}=M_{h, w}\left(s_{1}, s_{2}\right)+M_{h, w}\left(s_{3}, s_{4}\right), X_{2}=M_{h, w}\left(s_{1}, s_{3}\right)+$ $M_{h, w}\left(s_{2}, s_{4}\right)$ and $X_{3}=M_{h, w}\left(s_{1}, s_{4}\right)+M_{h, w}\left(s_{2}, s_{3}\right)$, so that $d_{h, w}$ satisfies the four points inequality (4.1) if $X_{1} \geq \min \left\{X_{2}, X_{3}\right\}$, which is a consequence of

$$
\#\left\{i \in\{1,2,3\}: X_{i}=\min \left(X_{1}, X_{2}, X_{3}\right)\right\} \geq 2 \text {. }
$$

To prove (4.26) it is convenient to work on $\left(T_{h}, d_{h}, r_{h}\right)$ that is the pointed compact real tree coded by $h$ as explained in Example 4.10: recall that $p_{h}:[0, \infty] \rightarrow T_{h}$ stands for the canonical projection and recall from (4.24) the definition of $w_{\sigma}$ and $\widehat{w}_{\sigma}$ for all $\sigma \in T_{h}$. For all $i \in\{1, \ldots, 4\}$, we set $\sigma_{i}=p_{h}\left(s_{i}\right)$ and we recall from (4.25) that $M_{h, w}\left(\sigma_{i}, \sigma_{j}\right)=M_{h, w}\left(s_{i}, s_{j}\right)$. Recall from (4.4) the definition of branch points in $T_{h}$. Since (4.26) does not depend on a specific indexation of the $s_{i}$, without loss of generality we can assume that $\gamma:=\operatorname{br}\left(\sigma_{1}, \sigma_{2}, \sigma_{3}\right)=\operatorname{br}\left(\sigma_{1}, \sigma_{2}, \sigma_{4}\right)$, that $\gamma^{\prime}=\operatorname{br}\left(\sigma_{3}, \sigma_{4}, \sigma_{1}\right)=\operatorname{br}\left(\sigma_{3}, \sigma_{4}, \sigma_{2}\right)$ and that

$$
a:=\min _{\sigma \in \llbracket \gamma, \sigma_{1} \rrbracket} \widehat{w}_{\sigma} \leq b:=\min _{\sigma \in \llbracket \gamma, \sigma_{2} \rrbracket} \widehat{w}_{\sigma}, \quad d:=\min _{\sigma \in \llbracket \gamma^{\prime}, \sigma_{3} \rrbracket} \widehat{w}_{\sigma} \leq e:=\min _{\sigma \in \llbracket \gamma^{\prime}, \sigma_{4} \rrbracket} \widehat{w}_{\sigma} \quad \text { and } \quad a \leq d .
$$

We also set $c:=\min _{\sigma \in \llbracket \gamma, \gamma^{\prime} \rrbracket} \widehat{w}_{\sigma}$. Then, $X_{1}=a+d, X_{2}=(a \wedge c)+(b \wedge c \wedge e)$ and $X_{3}=$ $(a \wedge c)+(b \wedge c \wedge d)$. We have four cases to consider; $(i)$ : if $c \leq a$, then $X_{1}=a+d \geq X_{2}=X_{3}=2 c$; (ii): if $a \leq c \leq d$, then $X_{1}=a+d \geq X_{2}=X_{3}=a+(b \wedge c)$; (iii): if $d \leq c$ and $b \leq d$, then $X_{1}=a+d \geq X_{2}=X_{3}=a+b$; (iv): if $d \leq c$ and $d \leq b$, then $X_{1}=X_{3}=a+d \leq X_{2}=a+(b \wedge c \wedge e)$. This proves (4.26) and it completes the proof of the lemma.

Definition 4.23. Let $\zeta \in(0, \infty)$ and let $(h, w) \in \boldsymbol{\Sigma}([0, \zeta])$. Let $d_{h, w}$ be the associated snake metric (as in Definition 4.19). Since $d_{h, w} \in \mathbf{M T}([0, \zeta])$ by Lemma 4.22, we denote by $T_{h, w}=[0, \zeta] / \sim_{d_{h, w}}$ the corresponding real tree and to simplify we denote by $p_{h, w}:[0, \zeta] \rightarrow$ $T_{h, w}$ the canonical projection, by $r_{h, w}=p_{h, w}(0)$ the root of $T_{h, w}$, and by $\mu_{h, w}$ the measure on $T_{h, w}$ induced by the Lebesgue measure on $[0, \zeta]$ via $p_{h, w}$ : namely,

$$
\int_{T_{h, w}} f(x) \mu_{h, w}(d x)=\int_{0}^{\zeta} f\left(p_{h, w}(s)\right) d s,
$$

for all continuous $f: T_{h, w} \rightarrow \mathbb{R}$. Since the pseudo-metric $d_{h, w}:[0, \zeta]^{2} \rightarrow[0, \infty)$ is continuous, so is $p_{h, w}$ and $\left(T_{h, w}, d_{h, w}, r_{h, w}, \mu_{h, w}\right)$ is a pointed measured compact real tree that we call the snake tree associated with $(h, w)$. By Lemma 4.20 and Proposition $4.14,(h, w) \in$ $\boldsymbol{\Sigma}([0, \zeta]) \mapsto T_{h, w} \in \mathbb{M}$ is $\left(\Delta, \boldsymbol{\delta}_{\mathrm{GHP}}\right)$-continuous.

Remark 4.24. Let $(h, w) \in \boldsymbol{\Sigma}([0, \zeta])$ and $\alpha, a, b \in(0, \infty)$. For all $s \in[0, b \zeta]$, we set $h^{\prime}(s)=\alpha h(s / b)$ and $w_{s}^{\prime}(r)=a w_{s / b}(r / \alpha)$. Then $\left(h^{\prime}, w^{\prime}\right) \in \boldsymbol{\Sigma}([0, b \zeta])$ and thanks to Remark 4.5, we easily check that $\left(T_{h^{\prime}}, d_{h^{\prime}}, r_{h^{\prime}}, \mu_{h^{\prime}}\right)$ is isometric to $\left(T_{h}, \alpha d_{h}, r_{h}, b \mu_{h}\right)$ and that $\left(T_{h^{\prime}, w^{\prime}}, d_{h^{\prime}, w^{\prime}}, r_{h^{\prime}, w^{\prime}}, \mu_{h^{\prime}, w^{\prime}}\right)$ is isometric to $\left(T_{h, w}, a d_{h, w}, r_{h, w}, b \mu_{h, w}\right)$.

Remark 4.25. Let $(h, w) \in \mathbf{\Sigma}([0, \zeta])$; let $\left(T_{h}, d_{h}, r_{h}, \mu_{h}\right)$ and $\left(T_{h, w}, d_{h, w}, r_{h, w}, \mu_{h, w}\right)$ be the compact real trees coded by resp. $h$ and $(h, w)$ and recall that $p_{h}$ and $p_{h, w}$ stand for the canonical projections from $[0, \zeta]$ to resp. $T_{h}$ and $T_{h, w}$. Observe that it actually makes sense to define a function y $: T_{h} \rightarrow T_{h, w}$ by setting

$$
\forall s \in[0, \zeta], \quad \mathrm{y}\left(p_{h}(s)\right)=p_{h, w}(s) .
$$


It is easy to deduce from (4.25) that

$$
d_{h, w}\left(\mathrm{y}(\sigma), \mathrm{y}\left(\sigma^{\prime}\right)\right)=\widehat{w}_{\sigma}+\widehat{w}_{\sigma^{\prime}}-2 \min _{\gamma \in \llbracket \sigma, \sigma^{\prime} \rrbracket} \widehat{w}_{\gamma},
$$

It implies that $\mathrm{y}$ is continuous and surjective. Note that $\mu_{h, w}$ is the image of $\mu_{h}$ via y.

We next prove two results that deal with basic geometric properties of snake trees. The first one provides conditions for a snake tree to be a continuum real tree.

Lemma 4.26. Let $(h, w) \in \boldsymbol{\Sigma}([0, \zeta])$. We set

$$
U_{h, w}=\left\{s \in[0, \zeta]: h(s)>0 \quad \text { and } \quad \forall \varepsilon \in(0, h(s)), \inf _{r \in[h(s)-\varepsilon, h(s)]}^{w_{s}(r)<\widehat{w}_{s}}\right\}
$$

and we assume that $[0, \zeta] \backslash U_{h, w}$ is Lebesgue-negligible. We also assume that the tree $\left(T_{h}, d_{h}, r_{h}, \mu_{h}\right)$ coded by $h$ is a continuum real tree as defined in (4.6). Then, the snake tree $\left(T_{h, w}, d_{h, w}, r_{h, w}, \mu_{h, w}\right)$ is also a continuum real tree.

Proof. We set $V=U_{h, w} \cap p_{h}^{-1}\left(\operatorname{Lf}\left(T_{h}\right)\right)$. Since $[0, \zeta] \backslash U_{h, w}$ is Lebesgue-negligible and since $T_{h}$ is a continuum real tree, $[0, \zeta] \backslash V$ is Lebesgue-negligible. To conclude the proof we are going to show that $V \subset U$, where $U$ is defined in (4.7) with $d$ replaced by $d_{h, w}$, so that Lemma 4.8 implies the desired result. To that end, we fix $s \in V$ and $s^{\prime} \in[0, \zeta]$ be distinct from $s$. Suppose that $h(s)=m_{h}\left(s, s^{\prime}\right)$; if there is $s^{\prime \prime} \in\left(s \wedge s^{\prime}, s \vee s^{\prime}\right)$ such that $h\left(s^{\prime \prime}\right)>m_{h}\left(s, s^{\prime}\right)$, then $p_{h}(s) \in \llbracket r, p_{h}\left(s^{\prime \prime}\right) \llbracket$ which is impossible since $p_{h}(s)$ has to be a leaf of $T_{h}$. Therefore, if $h(s)=m_{h}\left(s, s^{\prime}\right)$, then $h\left(s^{\prime \prime}\right)=m_{h}\left(s, s^{\prime}\right)$ for all $s^{\prime \prime} \in\left[s \wedge s^{\prime}, s \vee s^{\prime}\right]$; thus $\left[s \wedge s^{\prime}, s \vee s^{\prime}\right] \subset p_{h}^{-1}\left(\left\{p_{h}(s)\right\}\right)$ and $\mu_{h}$ would have an atom at $p_{h}(s)$, which is impossible since $T_{h}$ is a continuum real tree. Thus, we have proved that $h(s)>m_{h}\left(s, s^{\prime}\right)$. Therefore $\min _{r \in\left[m_{h}\left(s, s^{\prime}\right), h(s)\right]} w_{s}(r)<\widehat{w}_{s}$ since $s \in U_{h, w}$. Next, we set $b=\min \left\{w_{s}(r) ; r \in\left[0, m_{h}\left(s, s^{\prime}\right)\right]\right\}$; by Definition $4.17(b), b$ is also equal to $\min \left\{w_{s^{\prime}}(r) ; r \in\left[0, m_{h}\left(s, s^{\prime}\right)\right]\right\}$. We also set $a=\min \left\{w_{s}(r) ; r \in\left[m_{h}\left(s, s^{\prime}\right), h(s)\right]\right\}$ and $a^{\prime}=\min \left\{w_{s^{\prime}}(r) ; r \in\left[m_{h}\left(s, s^{\prime}\right), h\left(s^{\prime}\right)\right]\right\}$. Then,

$$
\begin{aligned}
d_{h, w}(0, s)+d_{h, w}\left(s, s^{\prime}\right)-d_{h, w}\left(0, s^{\prime}\right) & =2 \widehat{w}_{s}+2 M_{h, w}\left(0, s^{\prime}\right)-2 M_{h, w}(0, s)-2 M_{h, w}\left(s, s^{\prime}\right) \\
& =2 \widehat{w}_{s}-2 a+2\left(a+\left(b \wedge a^{\prime}\right)-(b \wedge a)-\left(a \wedge a^{\prime}\right)\right) .
\end{aligned}
$$

We next check that $q:=a+\left(b \wedge a^{\prime}\right)-(b \wedge a)-\left(a \wedge a^{\prime}\right) \geq 0$. Indeed, if $a \leq a^{\prime}, q=\left(b \wedge a^{\prime}\right)-(b \wedge a) \geq 0$; if $b \leq a^{\prime} \leq a, q=a-a^{\prime} \geq 0$; if $a^{\prime} \leq b \leq a, q=a-b \geq 0$; if $a^{\prime} \leq a \leq b, q=0$. Since we have proved that $a<\widehat{w}_{s}$, we get $d_{h, w}(0, s)+d_{h, w}\left(s, s^{\prime}\right)>d_{h, w}\left(0, s^{\prime}\right)$. This implies that $V \subset U$, which completes the proof.

Recall from (4.5) the definition of the degree of a point in a real tree and recall that a point is a branch point if its degree is $\geq 3$. The following lemma provides conditions ensuring that snake trees have only binary branch points.

Lemma 4.27. Let $(h, w) \in \boldsymbol{\Sigma}([0, \zeta])$. Let $T_{h}$ and $T_{h, w}$ be the compact real trees coded by resp. $h$ and $(h, w)$. Recall from Remark 4.21 that $w$ can actually be defined on $T_{h}$. Let $\mathcal{D}$ be a countable dense set of points of $T_{h}$. We consider two cases. In the first case, we assume the following.

(a) $\widehat{w}_{0}=0$ and $\widehat{w}_{s} \in[0, \infty)$ for all $s \in[0, \zeta]$.

(b) For all distinct $\sigma, \sigma^{\prime} \in \mathcal{D}$ such that $\min _{\gamma \in \llbracket \sigma, \sigma^{\prime} \rrbracket} \widehat{w}_{\gamma}>0$, there is at most one $\sigma_{0} \in \rrbracket \sigma, \sigma^{\prime} \llbracket$ such that $\widehat{w}_{\sigma_{0}}=\min _{\gamma \in \llbracket \sigma, \sigma^{\prime} \rrbracket} \widehat{w}_{\gamma}$ and when there is one, it is never a branch point of $T_{h}$.

Then, for all $x \in T_{h, w} \backslash\left\{r_{h, w}\right\}, \operatorname{deg}(x) \in\{1,2,3\}$. In the second case, we assume the following 
$\left(a^{\prime}\right) \widehat{w}_{0}=0$.

$\left(b^{\prime}\right)$ For all distinct $\sigma, \sigma^{\prime} \in \mathcal{D}$, there is at most one $\sigma_{0} \in \rrbracket \sigma, \sigma^{\prime} \llbracket$ such that $\widehat{w}_{\sigma_{0}}=\min _{\gamma \in \llbracket \sigma, \sigma^{\prime} \rrbracket} \widehat{w}_{\gamma}$ and when there is one, it is never a branch point of $T_{h}$.

Then, for all $x \in T_{h, w}, \operatorname{deg}(x) \in\{1,2,3\}$.

Proof. Let us consider the first case. We assume $(a)$ and $(b)$. We shall argue by contradiction. To that end, suppose that $x_{0} \in T_{h, w} \backslash\left\{r_{h, w}\right\}$ is such that $\operatorname{deg}\left(x_{0}\right) \geq 4$. Recall from (4.27) in Remark 4.25 the definition of the continuous surjective function $\mathrm{y}: T_{h} \rightarrow T_{h, w}$. Then, choose $x_{1}, x_{2}, x_{3} \in \mathrm{y}(\mathcal{D})$ such that $r_{h, w}, x_{1}, x_{2}, x_{3}$ are in distinct connected components of $T_{h, w} \backslash\left\{x_{0}\right\}$. Let $\sigma_{0} \in T_{h}$ and $\sigma_{1}, \sigma_{2}, \sigma_{3} \in \mathcal{D}$ be such that $\mathrm{y}\left(\sigma_{i}\right)=x_{i}$, $i \in\{0,1,2,3\}$. We first claim that for all $i, j \in\{1,2,3\}$ distinct,

$$
0<d_{h, w}\left(r_{h, w}, x_{0}\right)=\widehat{w}_{\sigma_{0}}=\min _{\gamma \in \llbracket \sigma_{0}, \sigma_{i} \rrbracket} \widehat{w}_{\gamma} \leq \inf _{\gamma \in \llbracket \sigma_{i}, \sigma_{j} \rrbracket} \widehat{w}_{\gamma} .
$$

Proof of (4.30). Note that $\mathrm{y}\left(r_{h}\right)=r_{h, w}$. Since $x_{0} \neq r_{h, w}$, by $(a)$ and (4.28), we get $0<d_{h, w}\left(x_{0}, r_{h, w}\right)=\widehat{w}_{\sigma_{0}}+\widehat{w}_{r_{h}}-2 \min _{\llbracket r_{h}, \sigma_{0} \rrbracket} \widehat{w}=\widehat{w}_{\sigma_{0}}$. Fix $i \in\{1,2,3\}$. Since $x_{0} \in \rrbracket r_{h, w}, x_{i} \llbracket$, (4.28) implies $\widehat{w}_{\sigma_{i}}=d_{h, w}\left(r_{h, w}, x_{i}\right)=d_{h, w}\left(r_{h, w}, x_{0}\right)+d_{h, w}\left(x_{0}, x_{i}\right)$ and thus $\widehat{w}_{\sigma_{i}}=2 \widehat{w}_{\sigma_{0}}+$ $\widehat{w}_{\sigma_{i}}-2 \min _{\gamma \in \llbracket \sigma_{0}, \sigma_{i} \rrbracket} \widehat{w}_{\gamma}$, which implies the second equality in (4.30). We complete the proof of (4.30) by noting that $\llbracket \sigma_{i}, \sigma_{j} \rrbracket \subset \llbracket \sigma_{0}, \sigma_{i} \rrbracket \cup \llbracket \sigma_{0}, \sigma_{j} \rrbracket$.

For all $i \in\{1,2,3\}$, let $C_{i}$ be the connected component of $T_{h, w} \backslash\left\{x_{0}\right\}$ that contains $x_{i}$. By connectivity, there are $\gamma_{1}, \gamma_{2} \in \llbracket \sigma_{1}, \sigma_{2} \rrbracket$ such that $\mathrm{y}\left(\gamma_{1}\right)=\mathrm{y}\left(\gamma_{2}\right)=x_{0}$ and $\llbracket \sigma_{i}, \gamma_{i} \llbracket \subset \mathrm{y}^{-1}\left(C_{i}\right)$, $i \in\{1,2\}$. Since $\mathrm{y}\left(\gamma_{i}\right)=x_{0}, \widehat{w}_{\sigma_{0}}+\widehat{w}_{\gamma_{i}}-2 \min _{\gamma \in \llbracket \sigma_{0}, \gamma_{i} \rrbracket} \widehat{w}_{\gamma}=0$, which implies that $\widehat{w}_{\gamma_{i}}=\widehat{w}_{\sigma_{0}}$. By (4.30), we get $\widehat{w}_{\gamma_{1}}=\widehat{w}_{\gamma_{2}}=\min _{\gamma \in \llbracket \sigma_{1}, \sigma_{2} \rrbracket} \widehat{w}_{\gamma}$. Then Assumption $(b)$ implies that $\gamma_{1}=\gamma_{2}$.

We next introduce $\beta=\operatorname{br}\left(\sigma_{1}, \sigma_{2}, \sigma_{3}\right)$, the branch point of $\sigma_{1}, \sigma_{2}, \sigma_{3}$ as defined in (4.4). Without loss of generality we can assume that $\gamma_{1} \in \llbracket \sigma_{1}, \beta \rrbracket$ and thus $\gamma_{1} \in \llbracket \sigma_{1}, \sigma_{3} \rrbracket$. By connectivity, there is $\gamma_{3} \in \llbracket \sigma_{1}, \sigma_{3} \rrbracket$ such that $y\left(\gamma_{3}\right)=x_{0}$ and $\llbracket \sigma_{3}, \gamma_{3} \llbracket \subset \mathrm{y}^{-1}\left(C_{3}\right)$. It implies that $\widehat{w}_{\gamma_{1}}=\widehat{w}_{\gamma_{3}}=\widehat{w}_{\sigma_{0}}=\min _{\gamma \in \llbracket \sigma_{1}, \sigma_{3} \rrbracket} \widehat{w}_{\gamma}$. Then Assumption $(b)$ implies that $\gamma_{3}=\gamma_{1}=\gamma_{2}$. By definition, the subsets $\llbracket \sigma_{i}, \gamma_{i} \llbracket, i \in\{1,2,3\}$ are pairwise disjoints and therefore $\gamma_{3}$ is a branch point of $T_{h}$ (it is actually $\operatorname{br}\left(\sigma_{1}, \sigma_{2}, \sigma_{3}\right)$ ) such that $\widehat{w}_{\gamma_{3}}=\min _{\llbracket \sigma_{1}, \sigma_{3} \rrbracket} \widehat{w}_{\gamma}$ which is not possible by Assumption $(b)$. This proves the first point of the lemma by contradiction. The exact same arguments can be used to prove the second case of the lemma.

\subsection{Stable Lévy trees}

In this section that contains no new result, we briefly recall basic definitions and properties on stable Lévy trees. Lévy trees are a class of random compact metric spaces that have been introduced by Le Gall and Le Jan in [25] (and further studied in Le Gall and D. [14]) as the genealogy of continuous state branching processes. Among stable trees, Aldous' continuum random tree corresponds to the Brownian case (see Aldous [3] and here below). Stable trees (and more generally Lévy trees) are the scaling limit of Galton-Watson trees as recalled in Introduction (see also below).

More precisely, let $\gamma \in(1,2]$ be the index of a spectrally positive stable Lévy process $X=\left(X_{s}\right)_{s \in[0, \infty)}$ : namely, the law of $X$ is characterised by its Laplace exponent that is given by

$$
\forall s, \lambda \in[0, \infty), \quad \mathbf{E}\left[\exp \left(-\lambda X_{s}\right)\right]=\exp \left(s \lambda^{\gamma}\right) .
$$

Note that $X$ is a Brownian motion when $\gamma=2$ and we shall refer to this case as the Brownian case. As shown in [25] (see also [14], Chapter 1), there exists a continuous process $H(X)=\left(H_{s}(X)\right)_{s \in[0, \infty)}$ such that for any $s \in[0, \infty)$,

$$
H_{s}(X)=\lim _{\varepsilon \rightarrow 0} \frac{1}{\varepsilon} \int_{0}^{t} \mathbf{1}_{\left\{I_{s}^{r}<X_{r}<I_{s}^{r}+\varepsilon\right\}} d r
$$


where $I_{s}^{r}$ stands for $\inf _{r \leq u \leq s} X_{u}$ and the convergence in (4.31) holds in probability. The process $H(X)$ is the $\gamma$-stable height process. Note that in the Brownian case, $H(X)$ is simply the reflected Brownian motion $s \mapsto 2\left(X_{s}-I_{s}^{0}\right)$. From the scaling property of $X$ and from (4.31), we see that for any $r \in(0, \infty),\left(r^{(\gamma-1) / \gamma} H_{t / r}(X)\right)_{t \in[0, \infty)}$ has the same distribution as $H(X)$.

As in the discrete setting, the process $H(X)$ encodes a family of continuum real trees: each excursion of $H(X)$ above 0 corresponds to an excursion of $X$ above its infimum (it is obvious in the Brownian case) and each excursion of $H(X)$ above 0 corresponds to a single continuum real tree of the family. The scaling property of $H(X)$ yields the following definition for the normalised excursion of $H(X)$ that is provided in D. [11] (see p.1005): set $\ell_{1}=\max \left\{s \in[0,1]: X_{s}=I_{s}\right\}, r_{1}=\min \left\{s \in[1, \infty): X_{s}=I_{s}\right\}$ and $\zeta_{1}=r_{1}-\ell_{1}$. Then, we set:

$$
\forall s \in[0,1], \quad H_{s}:=\zeta_{1}^{-\left(1-\frac{1}{\gamma}\right)} H_{\ell_{1}+s \zeta_{1}}(X) .
$$

The process $H$ is taken as the definition of the normalised excursion of the $\gamma$-stable height process.

As shown in Theorem 1.4.4 in Le Gall and D. [14], for all $\alpha \in\left(0,1-\frac{1}{\gamma}\right)$, P-a.s. $H(X)$ is $\alpha$-locally Hölder-continuous. Thus, the same holds true for $H$ : namely,

$$
\text { for all } \alpha \in\left(0,1-\frac{1}{\gamma}\right) \text {, a.s. } H \text { is } \alpha \text {-Hölder continuous. }
$$

Normalised stable Lévy trees. We call normalised $\gamma$-stable Lévy tree the real tree

$$
\left(T_{H}, d_{H}, r_{H}, \mu_{H}\right)
$$

coded by the function $H$, as defined in (4.8). Recall from (4.9) that $h \mapsto d_{h}$ is a 4-Lipschitz function from $\mathbf{C}([0,1], \mathbb{R})$ to $\mathbf{C}\left([0,1]^{2}, \mathbb{R}\right)$, both equipped with uniform norms. Thus, $d_{H}$ is a measurable random element of $\mathbf{M T}([0,1])$ and by Proposition 4.14, $T_{H}$ is a measurable random element of the Polish space ( $\left.\mathbb{M}, \boldsymbol{\delta}_{\mathrm{GHP}}\right)$ as mentioned in (4.11).

Let us briefly recall some geometric properties of $T_{H}$. One can prove that a.s. $T_{H}$ is a continuum real tree (as defined in (4.6)) and that the set of its branch points is a countable dense set; moreover we recall from Theorem 4.6 in Le Gall and D. [13] (p. 583) the following result.

$$
\text { A.s. for all } \sigma \in T_{H}, \operatorname{deg}(\sigma) \in A_{\gamma} \text {, where } A_{\gamma}= \begin{cases}\{1,2, \infty\} & \text { if } \gamma \in(1,2) \text {, } \\ \{1,2,3\} & \text { if } \gamma=2 .\end{cases}
$$

The contour of a discrete trees. We briefly recall how to code discrete trees by various functions. Let $t \in \mathbb{T}$ be a finite (rooted ordered) tree as in Definition 2.1. To simplify, we set $\# t=n$; since $t$ is finite, we can list the vertices of $t$ in the lexicographical order: $u_{0}=\varnothing<_{t} u_{1}<_{t} \ldots<_{t} u_{n-1}$; for all $j \in\{0, \ldots, n-1\}$, we set $H_{j}(t)=\left|u_{j}\right|$ that is the height of the $j$-th vertex of $t$. By convenience we also set $H_{n}(t)=0$. The function $\left(H_{k}(t)\right)_{0 \leq k \leq n}$ is the discrete height function of $t$. Note that $H(t)$ entirely encodes $t$.

We also introduce another coding function known as the contour (or the depth-first exploration) function of $t$. Informally, we embed $t$ into the clockwise oriented half plane so that order on siblings corresponds to orientation; we think of a particle that visits the tree at unit speed, that starts at the root and that goes from the left to right, backtracking as less as possible; we denote by $v(k)$ the vertex visited at time $k$ during this depth-first exploration. The particle crosses exactly twice each edge of $t$ : once upwards and once downwards; so, the total time needed to go back to the root is $2 n-2$, that is twice the number of edges. Namely, $\{(v(k), v(k+1)) ; 0 \leq k \leq 2 n-2\}=\{(u, \overleftarrow{u}),(\overleftarrow{u}, u) ; u \in t \backslash\{\varnothing\}\}$ Then, for all $k \in\{0, \ldots, 2 n-2\}$, we set $C_{k}(t)=|v(k)|$ and we also set $C_{2 n-1}(t)=C_{2 n}(t)=0$. We call contour function of $t$ the linear interpolation of the $C_{k}(t)$ that we still denote by $\left(C_{s}(t)\right)_{s \in[0,2 n]}$. 
The discrete height function and the contour function of $t$ are related as follows. For all $0 \leq j \leq n$, we set $b_{j}=2 j-H_{j}(t)$; we easily check that $\left(b_{j}\right)$ is an increasing sequence from 0 to $2 n$. Moreover, for all $j<n-1$, observe that for all $s \in\left[b_{j}, b_{j+1}\right]$,

$$
C_{s}(t)= \begin{cases}H_{j}(t)-\left(s-b_{j}\right) & \text { If } s \in\left[b_{j}, b_{j+1}-1\right], \\ s-b_{j+1}+H_{j+1}(t) & \text { If } s \in\left[b_{j+1}-1, b_{j+1}\right]\end{cases}
$$

and that $C_{s}(t)=H_{n-1}(t)-\left(s-b_{n-1}\right)$ if $s \in\left[b_{n-1}, b_{n}\right]$.

The pointed measured compact real tree coded by the contour function of $t$ is described as follows: to simplify notation, we set $C_{s}=C_{2 s}(t), s \in[0, n]$ and we recall from Example 4.9 the definition of $\left(\widetilde{t}, \widetilde{d}_{\mathrm{gr}}^{t}\right)$, the compact real tree spanned by the graph-tree $t$. It is easy to check that the pointed compact measured real tree $\left(T_{C}, d_{C}, r_{C}, \mu_{C}\right)$ coded by $C$ is isometric to $\left(\widetilde{t}, \widetilde{d}_{\mathrm{gr}}^{t}, \varnothing, \widetilde{\mathrm{m}}\right)$ where $\widetilde{\mathrm{m}}=\delta_{\varnothing}+$ Length, where $\delta_{\varnothing}$ stands for the Dirac mass at the root $\varnothing$.

We next recall the following limit theorem from D. [11] (Theorem 3.1, p. 1006).

Theorem 4.28 (Theorem 3.1 [11]). We fix $\gamma \in(1,2]$. Let $\tau$ be a Galton-Watson tree whose offspring distribution satisfies $(\mathbf{H})$ as in (1.1). Then, there exists a nondecreasing sequence of positive real numbers $\left(a_{n}\right)_{n \in \mathbb{N}}$ that tends to $\infty$ such that

$$
\begin{aligned}
& \left(\frac{1}{a_{n}} H_{\lfloor n s\rfloor}(\tau) ; \frac{1}{a_{n}} C_{2 n s}(\tau)\right)_{s \in[0,1]} \text { under } \mathbf{P}(\cdot \mid \# \tau=n) \\
& \stackrel{\text { (law) }}{\longrightarrow}\left(H_{s}, H_{s}\right)_{s \in[0,1]}
\end{aligned}
$$

where $H$ is the normalised excursion of the $\gamma$-stable height process.

\subsection{One-dimensional reflected Brownian snakes}

In this section we briefly introduce normalised one-dimensional (reflected) Brownian snake with $\gamma$-stable branching mechanism (i.e. whose lifetime process is a $\gamma$-stable height process). For more details we refer to the monograph of Le Gall and D. [14] (Chapter 4, pp. 107-149).

Let $I$ be an interval of $\mathbb{R}$ whose interior is not empty. Let $\left(Z_{r}^{x}\right)_{r \in[0, \infty)}$ be an $I$-valued continuous Markov process starting at $Z_{0}^{x}=x \in I$. We shall restrict to the two following cases:

- either $I$ is $\mathbb{R}$ and $Z^{x}$ is a Brownian motion,

- or $I=[0, \infty)$ and $Z^{x}$ is a Brownian motion that is reflected at 0 .

Recall from Definition 4.17 that $\mathbf{C}_{0}$ stands for the space of continuous functions from $[0, \infty)$ to $\mathbb{R}$ equipped with (Polish) topology of uniform convergence on all compact subsets. We let $w \in \mathbf{C}_{0}$ be $I$-valued and we fix two nonnegative real numbers $b \geq a$. We then denote by $R_{a, b}\left(w, d w^{\prime}\right)$ the law on $\mathbf{C}_{0}$ of the process $W(\cdot)$ that is defined as follows:

- for all $r \in[0, a], W(r)=w(r)$;

- the process $(W(a+r))_{r \in[0, \infty)}$ has the same law as $\left(Z_{r \wedge(b-a)}^{w(a)}\right)_{r \in[0, \infty)}$.

It is clear that $(a, b, w) \longmapsto R_{a, b}\left(w, d w^{\prime}\right)$ is weakly continuous on $\mathbf{C}_{0}$.

We next fix $\zeta \in(0, \infty)$ and $h \in \mathbf{C}([0, \zeta],[0, \infty))$ such that $h(0)=h(\zeta)=0$. Recall from (4.8) the definition of $m_{h}(\cdot, \cdot)$. It is easy to check that one defines a $\mathbf{C}_{0}$-valued process $\left(W_{s}(\cdot)\right)_{s \in[0, \zeta]}$ by specifying its finite dimensional marginals as follows:

- $W_{0}(r)=0, r \in[0, \infty)$.

- For all $0 \leq s_{1} \leq \ldots \leq s_{p} \leq \zeta,\left(W_{s_{k}}\right)_{1 \leq k \leq p}$ has law

$R_{m_{h}\left(0, s_{1}\right), h\left(s_{1}\right)}\left(W_{0}, d w_{1}\right) R_{m_{h}\left(s_{1}, s_{2}\right), h\left(s_{2}\right)}\left(w_{1}, d w_{2}\right) \ldots R_{m_{h}\left(s_{p-1}, s_{p}\right), h\left(s_{p}\right)}\left(w_{p-1}, d w_{p}\right)$. 
When the $Z^{x}$ are Brownian motions, the resulting collection of $\mathbf{C}_{0}$-valued r.v. $\left(W_{s}\right)_{s \in[0, \zeta]}$ is a Brownian snake with lifetime process $h$ and initial position 0 . When the $Z^{x}$ are reflected Brownian motions, $W$ is a reflected Brownian snake with lifetime process $h$ and initial position 0 . The following $\mathbb{R}$-valued process

$$
\forall s \in[0, \zeta], \quad \widehat{W}_{s}=W_{s}(h(s)),
$$

is the endpoint process of the snake $W$.

Remark 4.29. If $\left(W_{s}\right)_{s \in[0, \zeta]}$ is a Brownian snake with lifetime process $h$ and initial position 0 , then clearly $\left(\left|W_{s}\right|\right)_{s \in[0, \zeta]}$ is a reflected Brownian snake with lifetime process $h$ and initial position 0 .

Let us discuss the regularity of Brownian snakes. First, denote by $M_{s_{1}, \ldots, s_{p}}^{h}$ the law on $\mathbf{C}_{0}^{p}$ defined by (4.35). It is easy to check that $\left(s_{1}, \ldots, s_{p} ; h\right) \in[0, \zeta]^{p} \times \mathbf{C}([0, \zeta], \mathbb{R}) \longmapsto M_{s_{1}, \ldots, s_{p}}^{h}$ is weakly continuous on $\mathbf{C}_{0}^{p}$. Although $h$ is continuous, the process $W$ may not be continuous in general. However, the arguments used in the proof of Proposition 4.4.1 in Le Gall and D. [14] (Chapter 4 p. 127) show that if $h$ is Hölder, then $W$ has a continuous modification. More precisely, for all $\zeta, c \in(0, \infty)$ and all $\alpha \in(0,1]$, denote by $\mathrm{Höl}_{c, \alpha}([0, \zeta])$ the set of functions $h \in \mathbf{C}([0, \zeta],[0, \infty))$ such that $h(0)=h(\zeta)=0$ and $\left|h(s)-h\left(s^{\prime}\right)\right| \leq c\left|s-s^{\prime}\right|^{\alpha}$, for all $s, s^{\prime} \in[0, \zeta]$. From the proof of Proposition 4.4.1 in Le Gall and D. [14], we easily get the following lemma.

Lemma 4.30. Let $\zeta \in(0, \infty)$ and let $h \in \mathrm{Hol}_{c, \alpha}([0, \zeta])$. Let $\left(W_{s}\right)_{s \in[0, \zeta]}$ be a Brownian snake (or a reflected Brownian snake) with lifetime process $h$ and initial position 0 . Then, for all $p \in(1, \infty)$,

$$
\forall s, s^{\prime} \in[0, \zeta], \quad \mathbf{E}\left[\sup _{r \in[0, \infty)}\left|W_{s}(r)-W_{s^{\prime}}(r)\right|^{p}\right] \leq c_{p}\left|s-s^{\prime}\right|^{\alpha p / 2},
$$

where $c_{p}=(2 p \sqrt{c} /(p-1))^{p} \mathbf{E}\left[\left|Z_{1}^{0}\right|^{p}\right]$. By Kolmogorov's criterion, $W$ admits a modification that is $\alpha / 2$-Hölder with respect to the distance $\delta_{\mathrm{u}}$ on $\mathbf{C}_{0}$ (see (4.18) for the definition of $\delta_{\mathrm{u}}$ ).

We now assume that $h$ is Hölder; we keep denoting $W$ the continuous version of the (possibly reflected) Brownian snake with lifetime process $h$ and we briefly discuss properties of $W$ for later use.

First observe that a.s. $(h, W)$ is a snake as in Definition $4.17(b)$. Then, note that for all $s \in[0, \zeta],\left(W_{s}(r)\right)_{r \in[0, \infty)}$ has the same distribution as $\left(Z_{r \wedge h(s)}^{0}\right)_{r \in[0, \infty)}$, which easily implies the following: for all bounded and measurable $F: \mathbf{C}_{0} \rightarrow \mathbb{R}$,

$$
\mathbf{E}\left[\int_{0}^{\zeta} F\left(W_{s}(\cdot)\right) d s\right]=\int_{0}^{\zeta} \mathbf{E}\left[F\left(\left(Z_{r \wedge h(s)}^{0}\right)_{r \in[0, \infty)}\right)\right] d r .
$$

To define the normalised (reflected) Brownian snake with $\gamma$-stable branching mechanism, we need to shows that the law of a (reflected) Brownian snake is a Borelmeasurable function of its lifetime process. More precisely, since $\mathrm{Höl}_{c, \alpha}([0, \zeta])$ is a compact subset of $\mathbf{C}([0, \zeta],[0, \infty))$ equipped with uniform convergence and since $\mathrm{Höl}_{c, \alpha}([0, \zeta]) \subset \mathrm{Höl}_{\zeta^{\alpha-\alpha^{\prime}} c^{\prime}, \alpha^{\prime}}([0, \zeta])$ for all $0<\alpha^{\prime} \leq \alpha$ and all $c^{\prime} \geq c>0$, the set of Hölder lifetime-functions Höl $:=\bigcup_{c \in(0, \infty)} \bigcup_{\alpha \in(0,1]} \mathrm{Höl}_{c, \alpha}([0, \zeta])$ is a countable union of compact subsets and therefore a Borel subset of $\mathbf{C}([0, \zeta],[0, \infty))$. For all $h \in \mathrm{Höl}$, denote by $P_{h}(d W)$ (resp. $P_{h}^{+}(d W)$ ) the law on $\mathbf{C}\left([0, \infty), \mathbf{C}_{0}\right)$ of the Brownian snake (resp. of the reflected Brownian snake) $W$ with lifetime-function $h$ and initial position 0 and extend $h \mapsto P_{h}$ (resp. $h \mapsto P_{h}^{+}$) to $\mathbf{C}\left([0, \zeta],[0, \infty)\right.$ ) trivially by taking $P_{h}$ (resp. $P_{h}^{+}$) equal to the Dirac mass at the null function if $h$ is not a Hölder lifetime-function. Now recall that for all integers $p \geq 1$ and for all bounded continuous $F: \mathbf{C}_{0}^{p} \rightarrow \mathbb{R}$, the map 
$\left(s_{1}, \ldots, s_{p} ; h\right) \mapsto \int F\left(W_{s_{1}}, \ldots, W_{s_{p}}\right) P_{h}(d W)$ is continuous; the same holds with $P_{h}^{+}$. Since the Borel sigma field of $\mathbf{C}\left([0, \zeta], \mathbf{C}_{0}\right)$ equipped with $\Delta^{*}$ (as in Definition $4.17(a)$ ) is generated by the finite dimensional marginals, a standard monotone class argument implies that for all Borel subsets $B$ of the Polish space $\left(\mathbf{C}\left([0, \zeta], \mathbf{C}_{0}\right), \Delta^{*}\right)$, the map $h \in \mathbf{C}([0, \zeta],[0, \infty)) \longmapsto P_{h}(B) \in[0, \infty)$ is Borel-measurable. Namely:

$P_{h}(d W)$ is a Borel regular kernel from $\mathbf{C}([0, \zeta],[0, \infty))$ equipped with the uniform norm to the Polish space $\left(\mathbf{C}\left([0, \zeta], \mathbf{C}_{0}\right), \Delta^{*}\right)$. The same holds with $P_{h}^{+}$.

Definition 4.31. Let $\gamma \in(1,2]$. Let $H$ be the normalised excursion of the $\gamma$-stable height process. Recall from (4.32) that $H$ is a.s. Hölder-continuous. Thus, it makes sense to define the Brownian snake (resp. reflected Brownian snake) with $\gamma$-stable branching mechanism as the $\mathbf{C}([0, \zeta], \mathbb{R}) \times \mathbf{C}\left([0, \zeta], \mathbf{C}_{0}\right)$-valued r.v. $(H, W)$ whose regular conditional distribution given $H$ is $P_{H}(d W)$ (resp. $P_{H}^{+}(d W)$ ), as defined previously.

Let $(H, W)$ be a normalised Brownian snake with $\gamma$-stable branching mechanism. Recall that the real tree $\left(T_{H}, d_{H}, r_{h}, \mu_{H}\right)$ coded by $H$ is the $\gamma$-stable Lévy tree. Since a.s. $(H, W) \in \boldsymbol{\Sigma}([0,1]),(4.24)$ in Remark 4.21 applies to $(H, W)$ and it makes sense (up to a slight abuse of notation) to define $W$ and $\widehat{W}$ on $T_{H}$. To prove geometric properties of the $\gamma$-stable (reflected) Brownian cactus, we shall need the following result that is recalled from Lemma 6.4 in Le Gall and D. [13] (p. 600).

Lemma 4.32 (Lemma 6.4 [13]). Let $(H, W)$ be a normalised Brownian snake with $\gamma$ stable branching mechanism. Then, conditionally given $H,\left(\widehat{W}_{\sigma}\right)_{\sigma \in T_{H}}$ is a centered Gaussian process whose covariance is characterised by the following:

$$
\forall \sigma, \sigma^{\prime} \in T_{H}, \quad \int P_{H}(d W)\left|\widehat{W}_{\sigma^{\prime}}-\widehat{W}_{\sigma}\right|^{2}=d_{H}\left(\sigma, \sigma^{\prime}\right) .
$$

Moreover, for all $\varepsilon \in(0,1 / 2)$, conditionally given $H, \sigma \in T_{H} \longmapsto \widehat{W}_{\sigma}$ is $\left(\frac{1}{2}-\varepsilon\right)$-Höldercontinuous.

\subsection{Reflected Brownian cactus with stable branching mechanism}

Definition 4.33. Let $\gamma \in(1,2]$ and let $(H, W)$ be a normalised Brownian (resp. reflected Brownian) snake with $\gamma$-stable branching mechanism as in Definition 4.31. Recall the notation $\boldsymbol{\Sigma}([0,1])$ from Definition $4.17(b)$ and recall that $(H, W) \in \boldsymbol{\Sigma}([0,1])$. The Brownian (resp. reflected Brownian) cactus with $\gamma$-stable mechanism is the pointed measured compact real tree $\left(T_{H, W}, d_{H, W}, r_{H, W}, \mu_{H, W}\right)$ as specified in Definitions 4.19 and 4.23 .

Recall from (4.6) the definition of continuum real trees. Recall from (4.5) the definition of the degree of a point in a real tree.

Proposition 4.34. Let $\gamma \in(1,2]$. Let $(H, W)$ be a normalised Brownian (resp. reflected Brownian) snake with $\gamma$-stable branching mechanism and let $T_{H, W}$ the corresponding cactus as in Definition 4.33. Then, the following holds true.

(i) $\left(T_{H, W}, d_{H, W}, r_{H, W}, \mu_{H, W}\right)$ is a.s. a continuum real tree.

(ii) A.s. for all $x \in T_{H, W} \backslash\left\{r_{H, W}\right\}, \operatorname{deg}(x) \in\{1,2,3\}$. Moreover, if $T_{H, W}$ is a reflected Brownian cactus, then $\operatorname{deg}\left(r_{H, W}\right)=\infty$ and if $T_{H, W}$ is a Brownian cactus, then $\operatorname{deg}\left(r_{H, W}\right)=1$.

Remark 4.35. As recalled in (4.33), when $\gamma \in(1,2)$, the $\gamma$-stable Lévy tree $T_{H}$ has infinite branch points: namely, there is a countable dense set of points $\sigma \in T_{H}$ such that $\operatorname{deg}(\sigma)=\infty$. However, note that the corresponding Brownian cactus $T_{H, W}$ has only binary branch points (except possibly at the root in the reflected case). 
Remark 4.36. As already mentioned, the Brownian Cactus has been introduced by N. Curien, J-F. Le Gall and G. Miermont in [7] to study planar maps: roughly speaking the Brownian cactus corresponds to the case of a quadratic branching mechanism $\gamma=2$ and the spatial motion of the snake is a (unreflected) linear Brownian motion. In this case they proved much finer geometrical results: a.s. the upper-local density for typical points is 4 (Proposition 5.1 [7] p. 364) and the Hausdorff dimension is 4 (Corollary 5.3 [7] p. 365). See also the article by J-F. Le Gall [22] where the level sets of the Brownian cactus are studied to derive results on the Brownian maps.

Proof of Proposition 4.34. To prove $(i)$, we use Lemma 4.26. Indeed recall that $\left(T_{H}, d_{H}, r_{H}, \mu_{H}\right)$ is a.s. a continuum real tree and recall from (4.29) the definition of the subset $U_{H, W} \subset[0,1]$. Recall that $\left(Z_{r}^{0}\right)_{r \in[0, \infty)}$ stands for a one-dimensional Brownian motion (resp. reflected Brownian motion) starting at 0 that is independent from $H$. We denote by $\ell$ the Lebesgue measure on $[0,1]$. Then, by (4.36), conditionally given $H$, we get a.s.

$$
\begin{aligned}
\int P_{H}(d W) \ell\left(U_{H, W}\right) & =\int_{0}^{1} \mathbb{1}_{\left\{H_{s}>0\right\}} P_{H}\left(\forall \varepsilon \in\left(0, H_{s}\right): \inf _{r \in\left[H_{s}-\varepsilon, H_{s}\right]} W_{s}(r)<\widehat{W}_{s}\right) d s \\
& =\int_{0}^{1} \mathbb{1}_{\left\{H_{s}>0\right\}} \mathbf{P}\left(\forall \varepsilon \in\left(0, H_{s}\right): \inf _{r \in\left[H_{s}-\varepsilon, H_{s}\right]} Z_{r_{s}}^{0} Z_{H_{s}}^{0} \mid H\right) d s=1 .
\end{aligned}
$$

Thus, a.s. $\ell\left([0,1] \backslash U_{H, W}\right)=0$ and Lemma 4.26 implies $(i)$.

We next use Lemma 4.27 to prove $(i i)$. Recall that (up to a slight abuse of notation) $W$ can be defined on $T_{H}$. We first consider the unreflected Brownian case and recall from Lemma 4.32 that conditionally given $H,\left(\widehat{W}_{\sigma}\right)_{\sigma \in T_{H}}$ is a centered Hölder-continuous Gaussian process whose covariance is characterised by (4.37). According to Remark 4.29, $|\widehat{W}|$ is the endpoint process of the $\gamma$-stable reflected Brownian snake. We now work conditionally given $H$.

Let $\mathcal{D}$ be a countable dense subset of $T_{H}$. Let $\sigma, \sigma^{\prime} \in \mathcal{D}$ be distinct; denote by $\phi:\left[0, d_{H}\left(\sigma, \sigma^{\prime}\right)\right] \rightarrow \llbracket \sigma, \sigma^{\prime} \rrbracket_{T_{H}}$ the isometry such that $\phi(0)=\sigma$ and $\phi\left(d_{H}\left(\sigma, \sigma^{\prime}\right)\right)=\sigma^{\prime}$. The covariance of $\widehat{W}$ combined with the fact that Brownian motion is reversible entails that the process $Z_{r}:=\widehat{W}_{\phi(r)}-\widehat{W}_{\sigma}, r \in\left[0, d_{H}\left(\sigma, \sigma^{\prime}\right)\right]$, has the same law conditionally given $H$ as a Brownian motion starting at 0 : it therefore a.s. reaches its infimum at a unique time $\left.r_{0} \in\right] 0, d_{H}\left(\sigma, \sigma^{\prime}\right)$ [ and the law of $r_{0}$ conditionally given $H$ is diffuse, which entails that a.s. $\phi\left(r_{0}\right)$ cannot be a branch point of $T_{H}$ since the set of branch points of $T_{H}$ are countable. The same holds true for $|\widehat{W}|$ on $\llbracket \sigma, \sigma^{\prime} \rrbracket_{T_{H}}$ if $\inf _{\gamma \in \llbracket \sigma, \sigma^{\prime} \rrbracket_{T_{H}}}\left|\widehat{W}_{\gamma}\right|>0$. Since $\mathcal{D}$ is countable, it implies that conditionally given $H, \widehat{W}$ satisfies Assumptions $\left(a^{\prime}\right)$ and $\left(b^{\prime}\right)$ in Lemma 4.27 and that $|\widehat{W}|$ satisfies Assumptions $(a)$ and $(b)$ in Lemma 4.27. This lemma then implies the first assertion of $(i i)$.

Let us complete the proof of $(i i)$. Recall from Remarks 4.25 that it is makes sense to define $\mathrm{y}^{+}: T_{H} \rightarrow T_{H,|W|}$ by setting $\mathrm{y}^{+}\left(p_{H}(s)\right)=p_{H,|W|}(s), s \in[0,1]$. Let $\sigma \in T_{H}$ be such that $\mathrm{y}^{+}(\sigma) \neq r_{H,|W|}$. Then, observe that $\left\{\gamma \in \llbracket r_{H}, \sigma \rrbracket_{T_{H}}:\left|\widehat{W}_{\gamma}\right|>0\right\}$ has infinitely many connected components: let $\gamma$ and $\gamma^{\prime}$ be in two distinct such connected components: by (4.28) we get $d_{H,|W|}\left(\mathrm{y}^{+}(\gamma), \mathrm{y}^{+}\left(\gamma^{\prime}\right)\right)=\widehat{W}_{\gamma}+\widehat{W}_{\gamma^{\prime}}=d_{H,|W|}\left(r_{H, W}, \mathrm{y}^{+}\left(\gamma^{\prime}\right)\right)+d_{H,|W|}\left(r_{H, W}, \mathrm{y}^{+}(\gamma)\right)$, which easily implies that $\mathrm{y}^{+}(\gamma)$ and $\mathrm{y}^{+}\left(\gamma^{\prime}\right)$ belong to distinct connected components of $T_{H,|W|} \backslash\left\{r_{H,|W|}\right\}$. It implies that a.s. $\operatorname{deg}\left(r_{H,|W|}\right)=\infty$.

We define similarly y: $T_{H} \rightarrow T_{H, W}$ by setting $\mathrm{y}\left(p_{H}(s)\right)=p_{H, W}(s), s \in[0,1]$. Let $x, x^{\prime}$ and $r_{H, W}$ be distinct points of $T_{H, W}$ and denote by $z$ their branching point; let $\sigma$ and $\sigma^{\prime} \in T_{H}$ be such that $\mathrm{y}(\sigma)=x$ and $\mathrm{y}\left(\sigma^{\prime}\right)=x^{\prime}$ and recall that $\mathrm{y}\left(r_{H}\right)=r_{H, W}$. Then

$$
\begin{aligned}
& 2 d_{H, W}\left(r_{H, W}, z\right)=d_{H, W}\left(r_{H, W}, x\right)+d_{H, W}\left(r_{H, W}, x^{\prime}\right)-d_{H, W}\left(x, x^{\prime}\right) \\
& \text { by } \stackrel{(4.28)}{=} 2 \min _{\gamma \in \llbracket \sigma, \sigma^{\prime} \rrbracket_{T_{H}}} \widehat{W}_{\gamma}-2 \min _{\gamma \in \llbracket r_{H}, \sigma \rrbracket_{T_{H}}} \widehat{W}_{\gamma}-2 \min _{\gamma \in \llbracket r_{H}, \sigma^{\prime} \rrbracket_{T_{H}}} \widehat{W}_{\gamma} \text {. }
\end{aligned}
$$




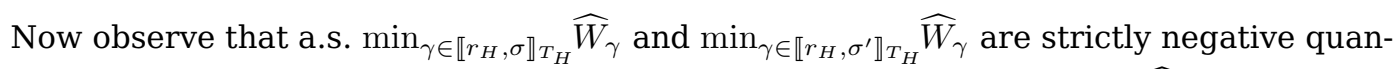
tities and that the least of the two has to be smaller than $\min _{\gamma \in \llbracket \sigma, \sigma^{\prime} \rrbracket_{T_{H}}} \widehat{W}_{\gamma}$. This prove that $d_{H, W}\left(r_{H, W}, z\right)>0$ and that $x$ and $x^{\prime}$ are in the same connected component of $T_{H, W} \backslash\left\{r_{H, W}\right\}$. Thus, $\operatorname{deg}\left(r_{H, W}\right)=1$, which completes the proof of $(i i)$.

\section{Scaling limit of the range of the BRW}

\subsection{Continuous interpolation of discrete snakes}

We briefly devise here a natural way to embed a tree-valued branching walk into a continuous branching motion, which takes its values in the real tree spanned by a discrete space-tree and is indexed by the real tree spanned by a genealogical tree.

To that end, let us consider a (possibly infinite) graph tree $T$ equipped with its graphdistance $d_{\mathrm{gr}}^{T}$. $T$ will be the space tree. We distinguish a special vertex $\circ \in T$ that plays the role of a root. Here, $T$ can be a rooted ordered tree as in Definition 2.1 or the b-ary tree $\mathbb{W}_{\mathrm{b}}$ or the free tree $\mathbb{W}_{[0,1]}$ as introduced in (2.1). Recall from Example 4.9 the definition of $\left(\widetilde{T}, \widetilde{d}_{\mathrm{g}^{T}}^{T}\right)$ of the real tree spanned by $T$. We make the necessary identifications to assume that $T \subset \widetilde{T}$.

We first explain how to obtain a continuous interpolation of a path $v(k) \in T, k \in$ $\{0, \ldots, n\}$ (by a path, we mean a sequence of adjacent vertices). It is easy to check that there exists a continuous map $\widetilde{v}:[0, n] \rightarrow \widetilde{T}$ such that $\widetilde{v}(k)=v(k)$ and for all $0 \leq k<n$,

$$
\forall s \in[k, k+1], \quad \widetilde{v}(s) \in \llbracket v(k), v(k+1) \rrbracket \text { is such that } \widetilde{d}_{\mathrm{gr}}^{T}(v(k), \widetilde{v}(s))=s-k .
$$

The continuous path $(\widetilde{v}(s))_{s \in[0, n]}$ is the continuous interpolation of the path $(v(k))_{k \in\{0, \ldots, n\}}$.

We next extend this interpolation to $T$-valued branching walks. Let $t \in \mathbb{T}$ be a finite (rooted ordered) tree as in Definition 2.1. $t$ will be the genealogical tree. We denote by $\left(\widetilde{t}, \widetilde{d}_{\mathrm{gr}}^{t}\right)$ the compact real tree spanned by $t$ and we assume that $t \subset \widetilde{t}$. Let $\left(Y_{v}\right)_{v \in t}$ be a $T$-valued branching walk indexed by $t$. Namely, for all $v \in t, Y_{v} \in T$ and it satisfies the following conditions: (a) $Y_{\varnothing}:=0$; (b) if $v, v^{\prime} \in t$ are neighbours, so are $Y_{v}$ and $Y_{v^{\prime}}$ in $T$. We easily check that there exists a continuous map $\widetilde{Y}: \widetilde{t} \mapsto \widetilde{T}$ such that $\widetilde{Y}_{v}=Y_{v}, v \in t$, by specifying the following: for all $\sigma \in \widetilde{t}$ there exist two neighbouring vertices $v, v^{\prime} \in t$ such that $\sigma \in \llbracket v, v^{\prime} \rrbracket_{\tilde{t}}$ and

$$
\widetilde{Y}_{\sigma} \text { is the only point of } \llbracket Y_{v}, Y_{v^{\prime}} \rrbracket_{\widetilde{T}} \text { such that } \widetilde{d}_{\mathrm{gr}}^{T}\left(Y_{v}, \widetilde{Y}_{\sigma}\right)=\widetilde{d}_{\mathrm{gr}}^{t}(v, \sigma) \text {. }
$$

We next introduce the spatial contour associated with $\left(Y_{v}\right)_{v \in t}$. To that end, denote by $v(k) \in t, 0 \leq k \leq 2 \# t$, the sequence of vertices of $t$ visited by the contour (or the depth-first) exploration of $t$ as recalled in (4.34) and note that they form a path in $t$. We denote by $\widetilde{v}:[0,2 \# t] \rightarrow \widetilde{t}$ its continuous interpolation. Observe that $C_{s}(t)=\widetilde{d}_{\mathrm{gr}}^{t}(\varnothing, \widetilde{v}(s))$, for all $s \in[0,2 \# t]$, where $C(t)$ stands for the contour function of $t$. Then, for all $s \in[0,2 \# t]$, we define a continuous map $V_{s}:[0, \infty) \rightarrow \widetilde{T}$ as follows:

$$
\left\{\begin{array}{l}
\text { for all } r \in\left[0, C_{s}(t)\right], V_{s}(r)=\widetilde{Y}_{\sigma} \text { where } \sigma \in \llbracket \varnothing, \widetilde{v}(s) \rrbracket_{\widetilde{t}} \text { is such that } r=\widetilde{d}_{\mathrm{gr}}^{t}(\varnothing, \sigma) ; \\
\text { for all } r \in\left[C_{s}(t), \infty\right), V_{s}(r)=\widetilde{Y}_{\widetilde{v}(s)} .
\end{array}\right.
$$

We call $\left(V_{s}(\cdot)\right)_{s \in[0,2 \# t]}$ the spatial contour associated with the branching walk $\left(Y_{v}\right)_{v \in t}$. It is easy to check here that $s \mapsto V_{s}$ is continuous from $[0,2 \# t]$ to the space of $\widetilde{T}$-valued continuous functions equipped with the uniform distance. We also define its endpoint process as follows:

$$
\forall s \in[0,2 \# t], \quad \widehat{V}_{s}=V_{s}\left(C_{s}(t)\right) .
$$


We then define the continuous interpolation of the discrete snake associated with $\left(Y_{v}\right)_{v \in t}$ and its endpoint process by

$$
\forall s \in[0,2 \# t], \quad \mathcal{W}_{s}(r)=\widetilde{d}_{\mathrm{gr}}^{T}\left(\mathrm{o}, V_{s}(r)\right), r \in[0, \infty) \quad \text { and } \quad \widehat{\mathcal{W}}_{s}=\widetilde{d}_{\mathrm{gr}}^{T}\left(\mathrm{o}, \widehat{V}_{s}\right) .
$$

By Remark 5.1 below, $\left(\mathcal{W}_{s}(\cdot)\right)_{s \in[0,2 \# t]}$ is the continuous interpolation of the $\mathbb{N}$-valued branching walk $\left(d_{\mathrm{gr}}^{T}\left(\mathrm{o}, Y_{v}\right)\right)_{v \in t}$. Moreover, observe that $\mathcal{W}$ is a snake whose lifetime function is the contour $C(t)$ of $t$ as in Definition 4.17: namely, $\left(C_{s}(t), \mathcal{W}_{s}\right)_{s \in[0,2 \# t]} \in$ $\boldsymbol{\Sigma}([0,2 \# t])$.

Remark 5.1. Let $T^{\prime}$ be another graph-tree and let $\phi: T \rightarrow T^{\prime}$ be an adjacency-preserving map so that the image by $\phi$ of a path in $T$ is a path in $T^{\prime}$ (for instance, $T=\mathbb{W}_{[0,1]}$, $T^{\prime}=\mathbb{W}_{\mathrm{b}}$ and $\phi=\Phi_{\mathrm{b}}$, the b-contraction as in Definition 3.3; or $T^{\prime}=\mathbb{N}$ and $\left.\phi(v)=d_{\mathrm{gr}}(\mathrm{o}, v)\right)$ Consequently, $\left(Y_{v}^{\prime}\right)_{v \in t}:=\left(\phi\left(Y_{v}\right)\right)_{v \in t}$ is a $T^{\prime}$-valued branching walk indexed by $t$. Then, $\phi$ clearly extends to a continuous map from $\widetilde{T} \rightarrow \widetilde{T}^{\prime}$ by requiring that $\widetilde{d}_{\mathrm{gr}}^{T^{\prime}}(\phi(\sigma), \phi(u))=$ $\widetilde{d}_{\mathrm{gr}}^{T}(\sigma, u)$ for all $\sigma \in \llbracket u, v \rrbracket_{\widetilde{T}}$ with $u$ and $v$ adjacent in $T$. Then, observe that the continuous interpolation of $Y^{\prime}$ is the image by $\phi$ of the continuous interpolation of $Y$ : namely, $\widetilde{Y}^{\prime}=\phi(\widetilde{Y})$.

We next provide basic properties related to counting and occupation measures on the range of $Y$ and its interpolation $\widetilde{Y}$. We first set

$$
\mathcal{R}=\left\{Y_{v} ; v \in t\right\} \quad \text { and } \quad \widetilde{\mathcal{R}}=\left\{\widehat{V}_{s} ; s \in[0,2 \# t]\right\}=\left\{\widetilde{Y}_{\sigma} ; \sigma \in \widetilde{t}\right\} .
$$

Observe that $\mathcal{R}$ (resp. $\widetilde{\mathcal{R}}$ ) is a subtree of $T$ (resp. $\widetilde{T}$ ) and note that $\widetilde{\mathcal{R}}$ is the real tree spanned by $\mathcal{R}$. We equip $\mathcal{R}$ and $\widetilde{\mathcal{R}}$ with the respective occupation measures $\mathbf{m}_{\text {cont }}$ and $\widetilde{\mathbf{m}}_{\text {cont }}$ of the endpoint process of the spatial contour $\widehat{V}$ (here, the subscript cont is for contour). Namely, for all bounded measurable $f: \widetilde{\mathcal{R}} \rightarrow \mathbb{R}$,

$$
\int_{\mathcal{R}} f d \mathbf{m}_{\text {cont }}=\sum_{0 \leq k<2 \# t} f\left(\widehat{V}_{k}\right) \text { and } \int_{\widetilde{\mathcal{R}}} f d \widetilde{\mathbf{m}}_{\text {cont }}=\int_{0}^{2 \# t} f\left(\widehat{V}_{s}\right) d s .
$$

We next introduce the spatial contour pseudo-distance $d_{\text {cont }}$ associated with $Y$ :

$$
\forall s, s^{\prime} \in[0,2 \# t], \quad d_{\text {cont }}\left(s, s^{\prime}\right)=\widetilde{d}_{\mathrm{gr}}^{T}\left(\widehat{V}_{s}, \widehat{V}_{s^{\prime}}\right) .
$$

Then, we easily check that $d_{\text {cont }} \in \mathbf{M T}([0,2 \# t])$ and that

the corresponding pointed measured compact real tree is isometric to $\left(\widetilde{\mathcal{R}}, \widetilde{d}_{\mathrm{gr}}^{T}, \mathrm{\circ}, \widetilde{\mathbf{m}}_{\text {cont }}\right)$.

Lemma 5.2. We keep the above notation. We denote by $\widetilde{d}_{\text {Haus }}$ the Hausdorff distance on the compact subsets of $\left(\widetilde{T}, \widetilde{d}_{\mathrm{g}}^{T}\right)$ and we denote by $\widetilde{d}_{\text {Prok }}$ the Prokhorov distance on the finite Borel measures on $\left(\widetilde{T}, \widetilde{d}_{\mathrm{gr}}^{T}\right)$. Then, $\widetilde{d}_{\text {Haus }}(\mathcal{R}, \widetilde{\mathcal{R}}) \leq 1, \widetilde{d}_{\text {Prok }}\left(\widetilde{\mathbf{m}}_{\text {cont }}, \mathbf{m}_{\text {cont }}\right) \leq 1$. Moreover, set $\mathbf{m}_{\mathrm{occ}}=\sum_{v \in t} \delta_{Y_{v}}$, that is the occupation measure of $Y$. Then, we also get $\widetilde{d}_{\text {Prok }}\left(\mathbf{m}_{\text {cont }}, 2 \mathbf{m}_{\text {occ }}\right) \leq 1$ and thus,

$$
\widetilde{d}_{\text {Prok }}\left(\widetilde{\mathbf{m}}_{\text {cont }}, 2 \mathbf{m}_{\text {occ }}\right) \leq 2 .
$$

Proof. Clearly, $\widetilde{d}_{\text {Haus }}(\mathcal{R}, \widetilde{\mathcal{R}}) \leq 1$. Next denote by $\pi$ the image of the Lebesgue measure on $[0,2 \# t]$ on $\widetilde{T} \times \widetilde{T}$ via the map $s \mapsto\left(\widehat{V}_{\lfloor s\rfloor}, \widehat{V}_{s}\right)$. By the definition (5.6), the first marginal of $\pi$ is $\mathbf{m}_{\text {cont }}$ and the second one is $\widetilde{\mathbf{m}}_{\text {cont }}$. Moreover by definition of the interpolation and of the spatial contour $\widetilde{d}_{g r}^{T}\left(\widehat{V}_{\lfloor s\rfloor}, \widehat{V}_{s}\right) \leq 1$. This easily implies that $\widetilde{d}_{\text {Prok }}\left(\widetilde{\mathbf{m}}_{\text {cont }}, \mathbf{m}_{\text {cont }}\right) \leq 1$.

To prove (5.9), we use the following coupling: recall that $(v(k))_{0 \leq k \leq 2 \# t}$ stands for the contour (or depth-first) exploration of $t$ (and recall the convention $v(2 \# t-1)=v(2 \# t)=\varnothing$ ). For all $k \in\{0, \ldots, 2 \# t-3\}$, we set $\rho(k)=v(k)$ if $|v(k)|=1+|v(k+1)|$ and $\rho(k)=v(k+1)$ 
if $|v(k+1)|=1+|v(k)|$ and we also set $\rho(2 \# t-2)=\rho(2 \# t-1)=\varnothing$. Note that the image measure of the counting measure on $\{0, \ldots, 2 \# t-1\}$ via $\rho$ is $2 \sum_{u \in t} \delta_{u}$, namely twice the counting measure on $t$ and thus, the image measure of the counting measure on $\{0, \ldots, 2 \# t-1\}$ via $Y_{\rho(\cdot)}$ is $2 \mathbf{m}_{\text {occ }}$. Then, denote by $\varpi$ the image measure on $T \times T$ of the counting measure on $\{0, \ldots, 2 \# t-1\}$ via the map $k \mapsto\left(Y_{\rho(k)}, \widehat{V}_{k}\right)$. We just have proved that the first marginal of $\varpi$ is $2 \mathbf{m}_{\text {occ }}$; the second marginal of $\varpi$ is by definition $\mathbf{m}_{\text {cont }}$. Since $Y_{\rho(k)}$ is either $\widehat{V}_{k}$ or $\widehat{V}_{k+1}$, we get $d_{\mathrm{gr}}^{T}\left(Y_{\rho(k)}, \widehat{V}_{k}\right) \leq 1$. This easily implies that $\widetilde{d}_{\text {Prok }}\left(\mathbf{m}_{\text {cont }}, 2 \mathbf{m}_{\text {occ }}\right) \leq 1$ and (5.9) follows immediately from the previous bound.

In the following lemma we provide bounds to compare the occupation measure induced by the spatial contour and the counting measure on $\mathcal{R}$.

Lemma 5.3. We keep the above notation. To simplify, we set $n=\# t$ and $n^{\prime}=\# \mathcal{R}$. We denote by $u_{0}, u_{1}, \ldots, u_{n-1}$ the sequence of the vertices of $t$ listed in the lexicographical order. Let $c \in(0, \infty)$. We set $\alpha=\max _{1 \leq i \leq n}\left|\#\left\{Y_{u_{j}} ; 0 \leq j<i\right\}-2 c i\right|$ and $\beta=\max _{s \in(0,2 n]}\left|\#\left\{\widehat{V}_{l} ; 0 \leq l<\lceil s\rceil\right\}-c s\right|$. Then,

$$
\beta \leq \alpha+3 c+c \max _{s \in[0,2 n]} C_{s}(t) .
$$

We next denote by $\mathbf{m}_{\text {count }}=\sum_{x \in \mathcal{R}} \delta_{x}$, the counting measure on $\mathcal{R}$ and for all $\eta \in(0, \infty)$, we set $q\left(d_{\text {cont }}, \eta\right)=\max \left\{d_{\text {cont }}\left(s, s^{\prime}\right) ; s, s^{\prime} \in[0,2 n]:\left|s-s^{\prime}\right| \leq \eta\right\}$, where $d_{\text {cont }}$ is defined by (5.7). Then,

$$
\widetilde{d}_{\text {Prok }}\left(\mathbf{m}_{\text {occ }}, \frac{n}{n^{\prime}} \mathbf{m}_{\text {count }}\right) \leq 1+2 q\left(d_{\text {cont }}, \frac{4 \beta+1}{c}\right) .
$$

Proof. By convenience we set $u_{n}=\varnothing$. Let $i \in\{0, \ldots, n-1\}$. Recall from the definition of the contour function of $t$ in (4.34) that $b_{i}:=2 i-\left|u_{i}\right|=\inf \left\{k \in\{0, \ldots, 2 n\}: v(k)=u_{i}\right\}$. Therefore, for all $k \in\left\{b_{i}+1, \ldots, b_{i+1}\right\},\{v(l) ; 0 \leq l<k\}=\left\{u_{j} ; 0 \leq j<i+1\right\}$ and we get

$\#\left\{\widehat{V}_{l} ; 0 \leq l<k\right\}=\#\left\{Y_{u_{j}} ; 0 \leq j<i+1\right\}=\#\left\{Y_{u_{j}} ; 0 \leq j<i+1\right\}-2 c(i+1)+c(2(i+1)-k)+c k$.

Note that $b_{i}<k \leq b_{i+1}$ implies that $|2(i+1)-k| \leq 2+\max _{0 \leq j \leq n}\left|u_{j}\right|=2+\max _{s \in[0,2 n]} C_{s}(t)$. Thus, we get

$$
\max _{1 \leq k \leq 2 n}\left|\#\left\{\widehat{V}_{l} ; 0 \leq l<k\right\}-c k\right| \leq \alpha+2 c+c \max _{s \in[0,2 n]} C_{s}(t),
$$

which immediately implies (5.10).

We next prove (5.11). To simplify set $J(k)=\#\left\{\widehat{V}_{l} ; 0 \leq l<k\right\}$, for all $k \in\{1, \ldots, 2 n\}$ and thus $\beta=\max _{s \in(0,2 n]}|J(\lceil s\rceil)-c s|$. For all $i^{\prime} \in\left\{1, \ldots, n^{\prime}\right\}$ we next set $k\left(i^{\prime}\right)=\inf \{k \in$ $\left.\{1, \ldots, 2 n\}: J(k)=i^{\prime}\right\}$ and $Z_{i^{\prime}}=\widehat{V}_{k\left(i^{\prime}\right)-1}$. Note that the $Z_{i^{\prime}}$ are distinct and that $\mathcal{R}=$ $\left\{Z_{i^{\prime}} ; 1 \leq i^{\prime} \leq n^{\prime}\right\}$. For all $i^{\prime}, j^{\prime} \in\left\{1, \ldots, n^{\prime}\right\},\left|k\left(i^{\prime}\right)-k\left(j^{\prime}\right)\right| \leq c^{-1}\left(2 \beta+\left|i^{\prime}-j^{\prime}\right|\right)$ since $J\left(k\left(i^{\prime}\right)\right)=i^{\prime}$ and $J\left(k\left(j^{\prime}\right)\right)=j^{\prime}$ and by definition of $\beta$. Then we get the following.

$$
d_{\mathrm{gr}}^{T}\left(Z_{i^{\prime}}, Z_{j^{\prime}}\right)=d_{\text {cont }}\left(k\left(i^{\prime}\right)-1, k\left(j^{\prime}\right)-1\right) \leq q\left(d_{\text {cont }},\left|k\left(j^{\prime}\right)-k\left(i^{\prime}\right)\right|\right) \leq q\left(d_{\text {cont }}, \frac{2 \beta+\left|i^{\prime}-j^{\prime}\right|}{c}\right) .
$$

Next observe that for all $k\left(i^{\prime}\right) \leq k<k\left(i^{\prime}+1\right)$, we get $J(k)=i^{\prime}$ and

$$
\left.d_{\mathrm{gr}}^{T}\left(\widehat{V}_{k-1}, Z_{J(k)}\right)=d_{\text {cont }}\left(k-1, k\left(i^{\prime}\right)-1\right) \leq q\left(d_{\text {cont }}, k\left(i^{\prime}+1\right)-k\left(i^{\prime}\right)\right) \stackrel{\text { by }}{\leq} \leq .12\right) q\left(d_{\text {cont }}, \frac{2 \beta+1}{c}\right)
$$

Thus, we have proved that

$$
\max _{s \in(0,2 n]} d_{\mathrm{gr}}^{T}\left(\widehat{V}_{\lfloor s\rfloor}, Z_{J(\lceil s\rceil)}\right) \leq q\left(d_{\text {cont }}, \frac{2 \beta+1}{c}\right) .
$$


Next observe that $\left|n^{\prime}-2 c n\right| \leq \beta$ and that

$$
J_{\lceil s\rceil}-\left\lceil\frac{n^{\prime}}{2 n} s\right\rceil=J_{\lceil s\rceil}-c s-\frac{s}{2 n}\left(n^{\prime}-2 c n\right)+\frac{n^{\prime}}{2 n} s-\left\lceil\frac{n^{\prime}}{2 n} s\right\rceil .
$$

Thus, $\max _{s \in(0,2 n]}\left|J(\lceil s\rceil)-\left\lceil n^{\prime} s / 2 n\right\rceil\right| \leq 1+2 \beta$ and by (5.12), $d_{\mathrm{gr}}^{T}\left(Z_{J(\lceil s\rceil)}, Z_{\left\lceil n^{\prime} s / 2 n\right\rceil}\right) \leq$ $q\left(d_{\text {cont }}, \frac{4 \beta+1}{c}\right)$. This inequality combined with (5.13) implies that

$$
\max _{s \in(0,2 n]} d_{\mathrm{gr}}^{T}\left(\widehat{V}_{\lfloor s\rfloor}, Z_{\left\lceil n^{\prime} s / 2 n\right\rceil}\right) \leq 2 q\left(d_{\text {cont }}, \frac{4 \beta+1}{c}\right) .
$$

We then denote by $\pi$ the image on $T \times T$ of the Lebesgue measure on $[0,2 n]$ via the map $s \mapsto\left(\widehat{V}_{\lfloor s\rfloor}, Z_{\left\lceil n^{\prime} s / 2 n\right\rceil}\right)$. The first marginal of $\pi$ is by definition $\mathbf{m}_{\text {cont }}$ and we easily see that the second marginal of $\pi$ is $\frac{2 n}{n^{\prime}} \mathbf{m}_{\text {count }}$. By (5.14), it implies that $\widetilde{d}_{\text {Prok }}\left(\mathbf{m}_{\text {cont }}, \frac{2 n}{n^{\prime}} \mathbf{m}_{\text {count }}\right) \leq$ $2 q\left(d_{\text {cont }}, \frac{4 \beta+1}{c}\right)$. Recall from Lemma 5.2 that $\widetilde{d}_{\text {Prok }}\left(\mathbf{m}_{\text {cont }}, 2 \mathbf{m}_{\text {occ }}\right) \leq 1$. Thus, we get $\widetilde{d}_{\text {Prok }}\left(2 \mathbf{m}_{\text {occ }}, \frac{2 n}{n^{\prime}} \mathbf{m}_{\text {count }}\right) \leq 1+2 q\left(d_{\text {cont }}, \frac{4 \beta+1}{c}\right)$, which implies (5.11) by Remark 4.12.

The range of the branching random walk. We next discuss the connection between the graph-metric of the range of branching random walks and the associated snake metric. More precisely, let $t \in \mathbb{T}$ be a finite (rooted ordered) tree as in Definition 2.1 and let $\left(Y_{v}\right)_{v \in t}$ be a $\mathbb{W}_{[0,1]}$-valued branching random walk with law $Q_{\mathrm{t}}^{+}$as in Definition 3.1 (ii). Recall from (5.5) that $\mathcal{R}=\left\{Y_{v} ; v \in t\right\}$ that is a subtree of $\mathbb{W}_{[0,1]}$ as in Definition 2.3. We denote by $d_{\text {gr }}$ the graph distance in $\mathbb{W}_{[0,1]}$ and we recall from Corollary 3.6 the fundamental property that makes the range $\mathcal{R}$ tractable:

$$
\text { P-a.s. for all } u, v \in t, \quad d_{\mathrm{gr}}\left(Y_{u}, Y_{v}\right)=\left|Y_{u}\right|+\left|Y_{v}\right|-2 \min _{w \in \llbracket u, v \rrbracket}\left|Y_{w}\right| .
$$

Next, let $\left(\widetilde{t}, \widetilde{d}_{\mathrm{gr}}^{t}\right)$ and $\left(\widetilde{\mathrm{W}}_{[0,1]}, \widetilde{d}\right)$ be the real trees spanned by respectively $t$ and $\mathbb{W}_{[0,1]}$; with a slight abuse of notation, we suppose that $t \subset \widetilde{t}$ and that $\mathbb{W}_{[0,1]} \subset \widetilde{\mathbb{W}}_{[0,1]}$. Let $\widetilde{Y}$ be the continuous interpolation of $Y$ in $\widetilde{\mathbb{W}}_{[0,1]}$ as defined by (5.1) (with $(T, \circ)=\left(\mathbb{W}_{[0,1]}, \varnothing\right)$ ) and recall from (5.5) that $\widetilde{\mathcal{R}}=\left\{\widetilde{Y}_{\sigma} ; \sigma \in \widetilde{t}\right\}$ is the tree spanned by $\mathcal{R}$. We easily check that (5.15) extends to $\widetilde{\mathcal{R}}$. Namely,

$$
\text { P-a.s. for all } \sigma, \sigma^{\prime} \in \widetilde{t}, \quad \widetilde{d}\left(\widetilde{Y}_{\sigma}, \widetilde{Y}_{\sigma^{\prime}}\right)=\left|\widetilde{Y}_{\sigma}\right|+\left|\widetilde{Y}_{\sigma^{\prime}}\right|-2 \min _{\varsigma \in \llbracket \sigma, \sigma^{\prime} \rrbracket}\left|\widetilde{Y}_{\varsigma}\right| \text {. }
$$

Let us rewrite this formula in terms of the contour function $C(t)$ of $t$, the spatial contour $V$, its endpoint process $\widehat{V}$ as defined in (5.3), and its corresponding snake $\mathcal{W}$ as defined in (5.4). To that end, first recall from (5.7) the definition of the spatial contour pseudo-distance $d_{\text {cont }}$ and recall from (4.19) the notation $M_{C(t), \mathcal{W}}(\cdot, \cdot)$ and the definition of the snake distance $d_{C(t), \mathcal{W}}$ associated with the snake $(C(t), \mathcal{W})$. Then, (5.16) translates into the following: $\mathbf{P}$-a.s. for all $s_{1}, s_{2} \in[0,2 \# t]$,

$$
d_{\text {cont }}\left(s_{1}, s_{2}\right)=\widetilde{d}\left(\widehat{V}_{s_{1}}, \widehat{V}_{s_{2}}\right)=\widehat{\mathcal{W}}_{s_{1}}+\widehat{\mathcal{W}}_{s_{2}}-2 M_{C(t), \mathcal{W}}\left(s_{1}, s_{2}\right)=d_{C(t), \mathcal{W}}\left(s_{1}, s_{2}\right) .
$$

Recall from (5.6) the definition of the occupation measure $\widetilde{\mathbf{m}}_{\text {cont }}$ induced by the spatial contour. Thus, (5.8) and (5.17) imply that

$$
\text { P-a.s. }\left(\widetilde{\mathcal{R}}, \widetilde{d}, \varnothing, \widetilde{\mathbf{m}}_{\text {cont }}\right) \text { and }\left(T_{C(t), \mathcal{W}}, d_{C(t), \mathcal{W}}, r_{C(t), \mathcal{W}}, \mu_{C(t), \mathcal{W}}\right) \text { are isometric. }
$$

where we recall from (4.23) that $\left(T_{C(t), \mathcal{W}}, d_{C(t), \mathcal{W}}, r_{C(t), \mathcal{W}}, \mu_{C(t), \mathcal{W}}\right)$ stands for the pointed measured compact real tree coded by the pseudo-metric $d_{C(t), \mathcal{W}}$.

We next couple the free branching random walk $\left(Y_{v}\right)_{t \in t}$ with the $\mathbb{W}_{\mathrm{b}}$-valued one via the b-contraction application $\Phi_{\mathrm{b}}$ as in Definition 3.3: namely we set

$$
\forall v \in t, \quad Y_{v}^{\mathrm{b}}=\Phi_{\mathrm{b}}\left(Y_{v}\right), \quad \text { and } \quad \mathcal{R}_{\mathrm{b}}=\left\{Y_{v}^{\mathrm{b}} ; v \in t\right\} .
$$


By Remark 3.4, $Y^{\mathrm{b}}$ has law $Q_{\mathrm{t}}^{+\mathrm{b}}$ as defined in Definition $3.1(i)$. Observe that $\mathcal{R}_{\mathrm{b}}$ is a subtree of $\mathbb{W}_{\mathrm{b}}$ as in Definition 2.3. We next denote by $d^{\mathrm{b}}$ the graph distance in $\mathbb{W}_{\mathrm{b}}$ and we denote by $\left(\widetilde{\mathbb{W}}_{\mathrm{b}}, \widetilde{d}^{\mathrm{b}}\right)$ the real tree spanned by $\mathbb{W}_{\mathrm{b}}$; with a slight abuse of notation, we suppose that $\mathbb{W}_{\mathrm{b}} \subset \widetilde{\mathbb{W}}_{\mathrm{b}}$. We extend $\Phi_{\mathrm{b}}$ as a map from $\widetilde{\mathbb{W}}_{[0,1]}$ to $\widetilde{\mathbb{W}}_{\mathrm{b}}$ explained in Remark 5.1 and we set

$$
\forall s \in[0,2 \# t], \forall r \in\left[0, C_{s}(t)\right], \quad V_{s}^{\mathrm{b}}(r)=\Phi_{\mathrm{b}}\left(V_{s}(r)\right) \quad \text { and } \quad \widehat{V}_{s}^{\mathrm{b}}=V_{s}^{\mathrm{b}}\left(C_{s}(t)\right),
$$

where we recall that $\left(V_{s}(\cdot)\right)_{s \in[0,2 \# t]}$ stands for the spatial contour of $Y$ as defined in (5.2). Note that $\widehat{V}_{s}^{\mathrm{b}}=\Phi_{\mathrm{b}}\left(\widehat{V}_{s}\right)$. By Remark 5.1 , it turns out that $V^{\mathrm{b}}$ is the spatial contour associated with $Y^{\mathrm{b}}$. Then, denote by $\widetilde{\mathcal{R}}_{\mathrm{b}}$ the compact real tree spanned by $\mathcal{R}_{\mathrm{b}}$ in $\widetilde{\mathrm{W}}_{\mathrm{b}}$. Namely,

$$
\widetilde{\mathcal{R}}_{\mathrm{b}}=\left\{\widehat{V}_{s}^{\mathrm{b}} ; s \in[0,2 \# t]\right\}=\Phi_{\mathrm{b}}(\widetilde{\mathcal{R}}) .
$$

We next extend Lemma 3.7 as follows: since $\left(\widehat{V}_{s}^{\mathrm{b}}\right)_{s \in[0,2 \# t]}$ is the continuous interpolation of the path $\left(Y_{v(k)}^{\mathrm{b}}\right)_{0 \leq k \leq 2 \# t}$ in $\mathcal{R}_{\mathrm{b}}$, we easily derive the following from (3.17).

For all $s_{1}, s_{2} \in[0,2 \# t]$, there exists a nonnegative r.v. $G_{s_{1}, s_{2}}$ such that

$$
2 G_{s_{1}, s_{2}}=\widetilde{d}\left(\widehat{V}_{s_{1}}, \widehat{V}_{s_{2}}\right)-\widetilde{d}^{\mathrm{b}}\left(\widehat{V}_{s_{1}}^{\mathrm{b}}, \widehat{V}_{s_{2}}^{\mathrm{b}}\right) \text { and } \mathbf{P}\left(G_{s_{1}, s_{2}} \geq x\right) \leq \mathrm{b}^{2-x}, x \in[0, \infty)
$$

This combined with (5.18) shows that $T_{C(t), W}$ and $\mathcal{R}_{\mathrm{b}}$ are close in a rough sense. It turns out that it is sufficient for the proof of Theorem 1.2.

\subsection{Invariance principle for discrete snakes}

In this section we recall one important result due to Marzouk (Theorem 1 [29]) that is an invariance principle for real valued endpoint processes of discrete snakes. Here we concentrate on the one-dimensional case but we shall need actually a slightly stronger version that holds for path-valued snakes and not only for endpoint processes.

More precisely, let us fix $\gamma \in(1,2]$ and let $\tau$ be a Galton-Watson tree (as in Definition 2.2) whose offspring distribution $\mu$ satisfies $(\mathbf{H})$ as in (1.1). Conditionally given $\tau$, let $\left(Y_{v}\right)_{v \in \tau}$ be a $\mathbb{Z}$-valued branching random walk whose transition kernel $q\left(y, d y^{\prime}\right)$ is that of the simple symmetric random walk on $\mathbb{Z}$. Then recall from (5.4) the definition of $\left(\mathcal{W}_{s}(\cdot)\right)_{s \in[0,2 \# \tau]}$, the continuous interpolation of the discrete snake (here the tree $T$ is $\mathbb{Z}$ ). Then,

$$
\forall k \in\{0, \ldots, 2 \# t\}, \quad \widehat{\mathcal{W}}_{k}=Y_{v(k)},
$$

where $v(k) \in \tau, 0 \leq k \leq 2 \# \tau$, stands for the sequence of vertices of $\tau$ visited by the contour (or the depth-first exploration) of $\tau$. Then, the following result is a special case of Theorem 1 in Marzouk [29]:

Theorem 5.4 (Theorem 1 [29]). Let $\gamma, \mu, \tau$ and $\left(a_{n}\right)_{n \in \mathbb{N}}$ be as in Theorem 4.28. Conditionally given $\tau$, let $\left(Y_{v}\right)_{v \in \tau}$ be defined as above and let $\left(\widehat{\mathcal{W}}_{k}\right)_{0 \leq k \leq 2 \# \tau}$ be the endpoint process of the snake associated with $\left(Y_{v}\right)_{v \in \tau}$ as in (5.20). Then

$$
\left(\frac{1}{a_{n}} C_{2 n s}(\tau), \frac{1}{\sqrt{a_{n}}} \widehat{\mathcal{W}}_{\lfloor 2 n s\rfloor}\right)_{s \in[0,1]} \text { under } \mathbf{P}(\cdot \mid \# \tau=n) \underset{n \rightarrow \infty}{\stackrel{\text { (law) }}{\longrightarrow}}\left(H_{s}, \widehat{W}_{s}\right)_{s \in[0,1]}
$$

where $(H, W)$ is the normalised one dimensional Brownian snake with $\gamma$-stable branching mechanism and where $\widehat{W}_{s}=W_{s}\left(H_{s}\right), s \in[0,1]$, stands for its endpoint process.

As already mentioned this result actually holds for fairly more general spatial motions in $\mathbb{R}^{d}$. It extends earlier results by Janson and Marckert [18] who considered snakes whose genealogical tree are in the domain of attraction of the Brownian tree. We refer to Marzouk [29] for more details. We next derive from Theorem 5.4 the following proposition that holds for the path-valued continuous interpolation of the discrete snake $\left(\mathcal{W}_{s}(\cdot)\right)_{s \in[0,2 \# \tau]}$. 
Proposition 5.5. We keep the same notation and the same assumption as in Theorem 5.4. Recall from Definition 4.17 (a) the metric $\Delta$ that makes the space $\mathbf{C}([0,1], \mathbb{R}) \times \mathbf{C}\left([0,1], \mathbf{C}_{0}\right)$ Polish. Then, weakly on that space, the following convergence holds.

$$
\left(\left(\frac{1}{a_{n}} C_{2 n s}(\tau)\right)_{s \in[0,1]},\left(\frac{1}{\sqrt{a_{n}}} \mathcal{W}_{2 n s}\left(\frac{\dot{a}}{a_{n}}\right)\right)_{s \in[0,1]}\right) \text { under } \mathbf{P}(\cdot \mid \# \tau=n) \underset{n \rightarrow \infty}{\stackrel{\text { (law) }}{\longrightarrow}}(H, W) .
$$

By taking the absolute value, this results holds true for the snake reflected at 0 .

Proof. Let $k_{0}=0 \leq k_{1} \leq \ldots \leq k_{p} \leq 2 \# \tau$ be integers. Then for all $j \in\{0, \ldots, p-1\}$, set $b_{j}=\min _{k_{j} \leq \ell \leq k_{j+1}} C_{\ell}(\tau)$ and $b_{j}^{\prime}=C_{k_{j+1}}(\tau)-b_{j}$. By definition of the branching random walk and its associated snakes, the following holds true conditionally given $\tau$ (or $C(\tau)$ ).

(i) The paths $S(j):=\left(\mathcal{W}_{k_{j+1}}\left(\left(b_{j}+\ell\right) \wedge C_{k_{j+1}}(\tau)\right)-\mathcal{W}_{k_{j+1}}\left(b_{j}\right)\right)_{\ell \in \mathbb{N}}$ are (conditionally) independent.

(ii) The conditional law of $S(j)$ is that of a $\mathbb{Z}$-valued symmetric random walk, starting at 0 and stopped at time $b_{j+1}^{\prime}$.

Next observe that $\left(b, b^{\prime}, w, w^{\prime}\right) \in[0, \infty)^{2} \times \mathbf{C}_{0}^{2} \mapsto\left(w(r \wedge b)+w^{\prime}\left((r-b)_{+} \wedge b^{\prime}\right)-w^{\prime}(0)\right)_{r \in[0, \infty)}$ is continuous (recall from Definition 4.17 that $\mathbf{C}_{0}$ is equipped with the Polish topology of the convergence on all compact subsets of times, which corresponds for instance to the metric $\delta_{\mathrm{u}}$ given in (4.18)). This combined with $(i),(i i)$ and easy arguments on linear interpolation imply that for all real numbers $0 \leq s_{1} \leq \ldots \leq s_{p} \leq 1,\left(\mathbf{w}_{s_{j}}^{(n)}\right)_{1 \leq j \leq p}$ under $\mathbf{P}(\cdot \mid \# \tau=n)$ converges weakly to $\left(W_{s_{j}}\right)_{1 \leq j \leq p}$ on $\mathbf{C}_{0}^{p}$, where we have set $\mathbf{w}_{s}^{(n)}(\cdot)=$ $a_{n}^{-1 / 2} \mathcal{W}_{2 n s}\left(\cdot / a_{n}\right)$, for all $s \in[0,1]$.

We next prove the tightness of the rescaled snakes. A standard argument on linear interpolation shows that Theorem 5.4 implies that the continuous endpoint processes $\widehat{\mathbf{w}}^{(n)}$ under $\mathbf{P}(\cdot \mid \# \tau=n)$ converge weakly to the endpoint process $\widehat{W}$. This implies that

$$
\forall \varepsilon \in(0, \infty), \quad \lim _{\eta \rightarrow+} \sup _{n \geq 0} \mathbf{P}\left(\omega_{\eta}\left(\widehat{\mathbf{w}}^{(n)}\right) \geq \varepsilon \mid \# \tau=n\right)=0,
$$

where $\omega_{\eta}\left(\widehat{\mathbf{w}}^{(n)}\right)$ stands for the $\eta$-uniform modulus of continuity of $\widehat{\mathbf{w}}^{(n)}$ over $[0,1]$. We next denote by $\omega_{\eta}\left(\mathbf{w}^{(n)}\right)$ the $\eta$-uniform modulus of continuity of $\mathbf{w}^{(n)}$ with respect to the metric $\delta_{\mathrm{u}}$ on $\mathbf{C}_{0}$ as introduced in Lemma 4.18; this lemma asserts that $\omega_{\eta}\left(\mathbf{w}^{(n)}\right) \leq 2 \omega_{\eta}\left(\widehat{\mathbf{w}}^{(n)}\right)$. This, combined with the weak convergence of the finite dimensional marginals of $\mathbf{w}^{(n)}$, entails that the laws of $\mathbf{w}^{(n)}$ are tight on $\mathbf{C}\left([0,1], \mathbf{C}_{0}\right)$ and we easily get (5.21).

\subsection{Proof of Theorem 1.2}

Let us fix $\gamma \in(1,2]$ and let $\tau$ be a Galton-Watson tree (as in Definition 2.2) whose offspring distribution $\mu$ satisfies $(\mathbf{H})$ as in (1.1). Then recall from (4.34) the definition of the contour process $\left(C_{s}(\tau)\right)_{s \in[0,2 \# \tau]}$ of $\tau$. Conditionally given $\tau$, let $\left(Y_{v}\right)_{v \in \tau}$ be a $\mathbb{W}_{[0,1]^{-}}$ valued branching random walk with law $Q_{\tau}^{+}$as in Definition $3.1(i i)$. Recall from (5.2) the spatial contour $\left(V_{s}(\cdot)\right)_{s \in[0,2 \# \tau]}$ associated with $Y$, and recall from (5.4) the definition of $\left(\mathcal{W}_{s}(\cdot)\right)_{s \in[0,2 \# \tau]}$, the continuous interpolation of the discrete snake. We denote by $\left(\widetilde{\tau}, \widetilde{d}_{\mathrm{gr}^{\tau}}^{\tau}\right),\left(\widetilde{\mathbb{W}}_{[0,1]}, \widetilde{d}\right)$ and $\left(\widetilde{\mathbb{W}}_{\mathrm{b}}, \widetilde{d}^{\mathrm{b}}\right)$ be the real trees spanned by respectively $\tau, \mathbb{W}_{[0,1]}$ and $\mathbb{W}_{\mathrm{b}}$ we assume that $\tau \subset \widetilde{\tau}$, that $\mathbb{W}_{[0,1]} \subset \widetilde{\mathbb{W}}_{[0,1]}$ and that $\mathbb{W}_{\mathrm{b}} \subset \widetilde{\mathbb{W}}_{\mathrm{b}}$. We next couple the free branching random walk $\left(Y_{v}\right)_{t \in t}$ with the $\mathbb{W}_{\mathrm{b}}$-valued one via the b-contraction application $\Phi_{\mathrm{b}}$ as in Definition 3.3: namely we set $Y_{v}^{\mathrm{b}}=\Phi_{\mathrm{b}}\left(Y_{v}\right)$ for all $v \in \tau$. By Remark 3.4, $Y^{\mathrm{b}}$ has law $Q_{\mathrm{t}}^{+\mathrm{b}}$ as in Definition $3.1(i)$. We also set $V^{\mathrm{b}}=\Phi_{\mathrm{b}}(V)$ that is the spatial contour of $Y^{\mathrm{b}}$, according to Remark 5.1. We recall the following notation $\mathcal{R}=\left\{Y_{v} ; v \in \tau\right\}$, $\mathcal{R}_{\mathrm{b}}=\left\{Y_{v}^{\mathrm{b}} ; v \in \tau\right\}=\Phi_{d}(\mathcal{R}), \widetilde{\mathcal{R}}=\left\{\widehat{V}_{s} ; s \in[0,2 \# \tau]\right\}$ and $\widetilde{\mathcal{R}}_{\mathrm{b}}=\left\{\widehat{V}_{s}^{\mathrm{b}} ; s \in[0,2 \# \tau]\right\}=\Phi_{\mathrm{b}}(\widetilde{\mathcal{R}})$.

We trivially extend $C(\tau)$ and $\mathcal{W}$ on $[0, \infty)$ by taking $C_{s}(\tau)$ equal to 0 and by taking $\mathcal{W}_{s}$ equal to the null function for all $s \in[2 \# \tau, \infty)$. Let $\left(a_{n}\right)_{n \in \mathbb{N}}$ be as in Theorem 4.28. To 
simplify notation we set for all $s \in[0,1]$,

$$
\mathbf{h}_{n}(s)=\frac{1}{a_{n}} C_{2 n s}(\tau), \quad \mathbf{w}_{s}^{(n)}(r)=\frac{1}{\sqrt{a_{n}}} \mathcal{W}_{2 n s}\left(\frac{r}{a_{n}}\right), r \in[0, \infty) \quad \text { and } \quad \widehat{\mathbf{w}}_{s}^{(n)}=\frac{1}{\sqrt{a_{n}}} \widehat{\mathcal{W}}_{2 n s} .
$$

Observe that $\mathbf{P}(\cdot \mid \# \tau=n)$-a.s. $\left(\mathbf{h}_{n}, \mathbf{w}^{(n)}\right) \in \boldsymbol{\Sigma}([0,1])$ as in Definition 4.17 and we denote by $d_{\mathbf{h}_{n}}$ and $d_{\mathbf{h}_{n}, \mathbf{w}(n)}$ the corresponding tree and snake pseudo-distances as defined in resp. (4.8) and (4.19). In particular, observe that

$$
\text { P-a.s. for all } s_{1}, s_{2} \in[0, \infty), \quad \frac{1}{\sqrt{a_{n}}} \widetilde{d}\left(\widehat{V}_{2 n s_{1}}, \widehat{V}_{2 n s_{2}}\right)=d_{\mathbf{h}_{n}, \mathbf{w}^{(n)}}\left(s_{1}, s_{2}\right)
$$

Proposition 5.5 combined with Lemma 4.20 implies that weakly on $\left(\mathbf{C}\left([0,1]^{2}, \mathbb{R}\right),\|\cdot\|\right)^{2}$

$$
\left(d_{\mathbf{h}_{n}}, d_{\mathbf{h}_{n}, \mathbf{w}^{(n)}}\right) \text { under } \mathbf{P}(\cdot \mid \# \tau=n) \underset{n \rightarrow \infty}{\longrightarrow}\left(d_{H}, d_{H, W}\right),
$$

where $(H, W)$ is the normalised one dimensional reflected Brownian snake with $\gamma$-stable branching mechanism as in Definition 4.31 and where $d_{H}$ and $d_{H, W}$ are the corresponding tree and snake pseudo-distances.

We next set

$$
\forall s_{1}, s_{2} \in[0, \infty), \quad \mathbf{d}_{n}^{*}\left(s_{1}, s_{2}\right)=\frac{1}{\sqrt{a_{n}}} \widetilde{d}^{\mathrm{b}}\left(\widehat{V}_{2 n s_{1}}^{\mathrm{b}}, \widehat{V}_{2 n s_{2}}^{\mathrm{b}}\right) .
$$

By (5.19), conditionally given $\tau, \mathbf{d}_{n}^{*}\left(s_{1}, s_{2}\right) \leq d_{\mathbf{h}_{n}, \mathbf{w}^{(n)}}\left(s_{1}, s_{2}\right)$ and for all $\varepsilon \in(0,1)$, a.s.

$$
\mathbf{P}\left(d_{\mathbf{h}_{n}, \mathbf{w}^{(n)}}\left(s_{1}, s_{2}\right)-\mathbf{d}_{n}^{*}\left(s_{1}, s_{2}\right)>2 \varepsilon \mid \tau\right) \leq \mathbf{b}^{3-\varepsilon \sqrt{a_{n}}},
$$

which implies that $\lim _{n \rightarrow \infty} \mathbf{P}\left(d_{\mathbf{h}_{n}, \mathbf{w}^{(n)}}\left(s_{1}, s_{2}\right)-\mathbf{d}_{n}^{*}\left(s_{1}, s_{2}\right)>2 \varepsilon \mid \# \tau=n\right)=0$. Therefore, Proposition 4.4 applies (with $\left.\mathbf{d}_{n}=d_{\mathbf{h}_{n}, \mathbf{w}^{(n)}}\right)$ to show that $\left(\mathbf{d}_{n}^{*}, d_{\mathbf{h}_{n}, \mathbf{w}^{(n)}}\right)$ under $\mathbf{P}(\cdot \mid \# \tau=$ $n)$ converge to $\left(d_{H, W}, d_{H, W}\right)$, weakly on $\left(\mathbf{C}\left([0,1]^{2}, \mathbb{R}\right),\|\cdot\|\right)^{2}$. Actually it is easy to see that $\left(d_{\mathbf{h}_{n}}, \mathbf{d}_{n}^{*}, d_{\mathbf{h}_{n}, \mathbf{w}}(n)\right)$ under $\mathbf{P}(\cdot \mid \# \tau=n)$ converges to $\left(d_{H}, d_{H, W}, d_{H, W}\right)$, weakly on $\left(\mathbf{C}\left([0,1]^{2}, \mathbb{R}\right),\|\cdot\|\right)^{3}$.

We next recall that $\mathbf{P}(\cdot \mid \# \tau=n)$-a.s. the real tree coded by $d_{\mathbf{h}_{n}}$ is isometric to $\left(\widetilde{\tau}, \frac{1}{a_{n}} \widetilde{d}_{g r}^{\tau}, \varnothing, \frac{1}{n} \widetilde{\mathrm{m}}\right)$ where $\widetilde{\mathrm{m}}=\delta_{\varnothing}+$ Length. Also recall from (5.8) that $\mathbf{P}(\cdot \mid \# \tau=n)$-a.s. the real tree coded by $\mathbf{d}_{n}^{*}$ is isometric to $\left(\widetilde{\mathcal{R}}_{\mathrm{b}}, \frac{1}{\sqrt{a_{n}}} \widetilde{d}^{\mathrm{b}}, \varnothing, \frac{1}{2 n} \widetilde{\mathbf{m}}_{\text {cont }}^{\mathrm{b}}\right)$, where $\widetilde{\mathbf{m}}_{\text {cont }}^{\mathrm{b}}$ is the occupation measure of $\left(\widehat{V}_{s}^{\mathrm{b}}\right)_{s \in[0,2 n]}$, as defined in general in (5.6). Since $\left(d_{\mathbf{h}_{n}}, \mathbf{d}_{n}^{*}\right)$ under $\mathbf{P}(\cdot \mid \# \tau=n)$ converges to $\left(d_{H}, d_{H, W}\right)$, weakly on $\left(\mathbf{C}\left([0,1]^{2}, \mathbb{R}\right),\|\cdot\|\right)^{2}$, Proposition 4.14 implies that the pointed measured compact spaces $\left(\widetilde{\tau}, \frac{1}{a_{n}} \widetilde{d}_{g^{r}}^{r}, \varnothing, \frac{1}{n} \widetilde{\mathrm{m}}\right)$ and $\left(\widetilde{\mathcal{R}}_{\mathrm{b}}, \frac{1}{\sqrt{a_{n}}} \widetilde{d}^{\mathrm{b}}, \varnothing, \frac{1}{2 n} \widetilde{\mathbf{m}}_{\text {cont }}^{\mathrm{b}}\right)$ under $\mathbf{P}(\cdot \mid \# \tau=n)$ jointly converge to resp. $\left(T_{H}, d_{H}, r_{H}, \mu_{H}\right)$ and $\left(T_{H, W}, d_{H, W}, r_{H, W}, \mu_{H, W}\right)$ weakly on $\left(\mathbb{M}, \boldsymbol{\delta}_{\mathrm{GHP}}\right)^{2}$, where $T_{H}$ and $T_{H, W}$ here stand for the real trees coded by resp. $H$ and $(H, W)$.

We next recall the following notation.

$$
\mathrm{m}=\sum_{v \in \tau} \delta_{v}, \mathbf{m}_{\mathrm{occ}}^{\mathrm{b}}=\sum_{v \in \tau} \delta_{Y_{v}^{\mathrm{b}}}, \overline{\mathbf{m}}_{\mathrm{occ}}^{\mathrm{b}}=\frac{1}{n} \mathbf{m}_{\mathrm{occ}}^{\mathrm{b}}, \mathbf{m}_{\text {count }}^{\mathrm{b}}=\sum_{x \in \mathcal{R}_{\mathrm{b}}} \delta_{x} \text { and } \overline{\mathbf{m}}_{\text {count }}^{\mathrm{b}}=\frac{1}{\# \mathcal{R}_{\mathrm{b}}} \mathbf{m}_{\text {count }}^{\mathrm{b}} .
$$

By the inequalities specified in Example 4.13 , we get $d_{\text {Haus }}(\tau, \widetilde{\tau}) \leq 1$ and $d_{\text {Prok }}(\widetilde{\mathrm{m}}, \mathrm{m}) \leq 3$. Similarly, by Lemma 5.2 we get $d_{\text {Haus }}\left(\mathcal{R}_{\mathrm{b}}, \widetilde{\mathcal{R}}_{\mathrm{b}}\right) \leq 1$ and $d_{\text {Prok }}\left(2 \mathbf{m}_{\text {occ }}^{\mathrm{b}}, \widetilde{\mathbf{m}}_{\text {cont }}^{\mathrm{b}}\right) \leq 2$. Thus,

$$
\begin{aligned}
& \left(\left(\tau, \frac{1}{a_{n}} d_{\mathrm{gr}}, \varnothing, \frac{1}{n} \mathrm{~m}\right),\left(\mathcal{R}_{\mathrm{b}}, \frac{1}{\sqrt{a_{n}}} d_{\mathrm{gr}}, \varnothing, \frac{1}{n} \mathbf{m}_{\mathrm{occ}}^{\mathrm{b}}\right)\right) \text { under } \mathbf{P}(\cdot \mid \# \tau=n) \\
& \underset{n \rightarrow \infty}{\longrightarrow}\left(\left(T_{H}, d_{H}, r_{H}, \mu_{H}\right),\left(T_{H, W}, d_{H, W}, r_{H, W}, \mu_{H, W}\right)\right),
\end{aligned}
$$

where $d_{\mathrm{gr}}$ stands for both graph-tree distances in $\tau$ and $\mathrm{W}_{\mathrm{b}}$ to simplify notation.

We next control $\mathbf{m}_{\text {count }}^{\mathrm{b}}$ in terms of $\mathbf{m}_{\mathrm{occ}}^{\mathrm{b}}$ thanks to Theorem 1.1 and Lemma 5.3. To that end, we set $c=c_{\mu, \mathrm{b}} / 2$ where $c_{\mu, \mathrm{b}}$ is as in Theorem 1.1; we denote by $u_{0}, u_{1}, \ldots, u_{\# \tau-1}$ the vertices of $\tau$ listed in the lexicographical order and we also set $\alpha=\max _{1 \leq i \leq \# \tau} \mid \#\left\{Y_{u_{j}}^{\mathrm{b}} ; 0 \leq\right.$ 
$j<i\}-2 c i \mid$ and $\beta=\max _{s \in(0,2 \# \tau]}\left|\#\left\{\widehat{V}_{l}^{\mathrm{b}} ; 0 \leq l<\lceil s\rceil\right\}-c s\right|$. Theorem 1.1 asserts that for all $\varepsilon \in(0, \infty), \mathbf{P}(\alpha / n>\varepsilon \mid \# \tau=n)$ tends to 0 as $n \rightarrow \infty$. By (5.10), $\mathbf{P}(\cdot \mid \# \tau=n)$-a.s.

$$
\frac{\beta}{n} \leq \frac{1}{n}(\alpha+3 c)+\frac{c a_{n}}{n} \max _{s \in[0,1]} h_{n}(s) .
$$

Since $\left(a_{n}\right)$ is $\frac{\gamma-1}{\gamma}$-regularly varying, $\lim _{n \rightarrow \infty} a_{n} / n=0$, which implies that $\mathbf{P}(\beta / n>\varepsilon \mid \# \tau=$ $n$ ) tends to 0 as $n \rightarrow \infty$.

Next, we denote by $d_{\text {Prok }}$ the Prokhorov distance on the space of finite measures on $\left(\mathcal{R}_{\mathrm{b}}, d_{\mathrm{gr}}\right)$ and we denote by $d_{\text {Prok }}^{(n)}$ the Prokhorov distance on the space of $\left(\mathcal{R}_{\mathrm{b}}, \frac{1}{\sqrt{a_{n}}} d_{\mathrm{gr}}\right)$. Since $n^{-1} \leq a_{n}^{-1 / 2}$ for all sufficiently large $n$, Remark 4.12 combined with (5.11) implies that $\mathbf{P}(\cdot \mid \# \tau=n)$-a.s.

$$
d_{\text {Prok }}^{(n)}\left(\overline{\mathbf{m}}_{\text {occ }}^{\mathrm{b}}, \overline{\mathbf{m}}_{\text {count }}^{\mathrm{b}},\right) \stackrel{\text { Remark } 4.12}{\leq} \frac{1}{\sqrt{a_{n}}} d_{\text {Prok }}\left(\mathbf{m}_{\text {occ }}^{\mathrm{b}}, \frac{n}{\# \mathcal{R}_{\mathrm{b}}} \mathbf{m}_{\text {count }}^{\mathrm{b}}\right) \stackrel{\text { by (5.11) }}{\leq} \frac{1}{\sqrt{a_{n}}}+2 q\left(\mathbf{d}_{n}^{*}, \frac{4 \beta+1}{2 n c}\right),
$$

where $q\left(\mathbf{d}_{n}^{*}, \eta\right)=\max \left\{\mathbf{d}_{n}^{*}\left(s, s^{\prime}\right) ; s, s^{\prime} \in[0,1]:\left|s-s^{\prime}\right| \leq \eta\right\}$. Since $\mathbf{d}_{n}^{*} \rightarrow d_{H, W}$ weakly on $\left(\mathbf{C}\left([0,1]^{2}, \mathbb{R}\right),\|\cdot\|\right)$ and since for all $\varepsilon \in(0, \infty), \lim _{n \rightarrow \infty} \mathbf{P}(\beta / n>\varepsilon \mid \# \tau=n)=0$, Proposition 4.3 implies that $\lim _{n \rightarrow \infty} \mathbf{P}\left(q\left(\mathbf{d}_{n}^{*}, \frac{4 \beta+1}{2 n c}\right)>\varepsilon \mid \# \tau=n\right)=0$ and thus

$$
\forall \varepsilon \in(0,1), \quad \lim _{n \rightarrow \infty} \mathbf{P}\left(d_{\text {Prok }}^{(n)}\left(\overline{\mathbf{m}}_{\text {occ }}^{\mathrm{b}}, \overline{\mathbf{m}}_{\text {count }}^{\mathrm{b}}\right)>\varepsilon \mid \# \tau=n\right)=0 .
$$

Then, observe that $\# \mathcal{R}_{\mathrm{b}} \leq n$ and that $\frac{\# \mathcal{R}_{\mathrm{b}}}{n} \overline{\mathbf{m}}_{\text {count }}^{\mathrm{b}}=\frac{1}{n} \mathbf{m}_{\text {count }}^{\mathrm{b}}$. By Remark 4.12, we get

$$
\begin{aligned}
d_{\text {Prok }}^{(n)}\left(c_{\mu, \mathrm{b}} \overline{\mathbf{m}}_{\text {occ }}^{\mathrm{b}}, \frac{1}{n} \mathbf{m}_{\text {count }}^{\mathrm{b}}\right) & \leq d_{\text {Prok }}^{(n)}\left(c_{\mu, \mathrm{b}} \overline{\mathbf{m}}_{\text {occ }}^{\mathrm{b}}, \frac{\# \mathcal{R}_{\mathrm{b}}}{n} \overline{\mathbf{m}}_{\text {occ }}^{\mathrm{b}}\right)+d_{\text {Prok }}^{(n)}\left(\frac{\# \mathcal{R}_{\mathrm{b}}}{n} \overline{\mathbf{m}}_{\text {occ }}^{\mathrm{b}}, \frac{\# \mathcal{R}_{\mathrm{b}}}{n} \overline{\mathbf{m}}_{\text {count }}^{\mathrm{b}}\right) \\
& \leq\left|\frac{\# \mathcal{R}_{\mathrm{b}}}{n}-c_{\mu, \mathrm{b}}\right|+d_{\text {Prok }}^{(n)}\left(\overline{\mathbf{m}}_{\text {occ }}^{\mathrm{b}}, \overline{\mathbf{m}}_{\text {count }}^{\mathrm{b}}\right) .
\end{aligned}
$$

Now, recall that $c_{\mu, \mathrm{b}}=2 c$ and that $\left|\frac{\# \mathcal{R}_{\mathrm{b}}}{n}-c_{\mu, \mathrm{b}}\right| \leq \alpha / n$. It finally implies that

$$
\forall \varepsilon \in(0,1), \quad \lim _{n \rightarrow \infty} \mathbf{P}\left(d_{\text {Prok }}^{(n)}\left(c_{\mu, \mathrm{b}} \overline{\mathbf{m}}_{\text {occ }}^{\mathrm{b}}, \frac{1}{n} \mathbf{m}_{\text {count }}^{\mathrm{b}}\right)>\varepsilon \mid \# \tau=n\right)=0,
$$

which completes the proof of Theorem 1.2.

\section{References}

[1] Romain Abraham, Jean-François Delmas, and Patrick Hoscheit, A note on the GromovHausdorff-Prokhorov distance between (locally) compact metric measure spaces, Electron. J. Probab. 18 (2013), no. 14, 21. MR3035742

[2] Elie Aïdékon and Loïc de Raphélis, Scaling limit of the recurrent biased random walk on a Galton-Watson tree, Probab. Theory Related Fields 169 (2017), no. 3-4, 643-666. MR3719055

[3] David Aldous, The continuum random tree. III, Ann. Probab. 21 (1993), no. 1, 248-289. MR1207226

[4] Itai Benjamini and Sebastian Müller, On the trace of branching random walks, Groups Geom. Dyn. 6 (2012), no. 2, 231-247. MR2914859

[5] Philippe Bougerol and Thierry Jeulin, Brownian bridge on hyperbolic spaces and on homogeneous trees, Probab. Theory Related Fields 115 (1999), no. 1, 95-120. MR1715541

[6] Xinxin Chen and Grégory Miermont, Long Brownian bridges in hyperbolic spaces converge to Brownian trees, Electron. J. Probab. 22 (2017), Paper No. 58, 15. MR3683367

[7] Nicolas Curien, Jean-François Le Gall, and Grégory Miermont, The Brownian cactus I. Scaling limits of discrete cactuses, Ann. Inst. Henri Poincaré Probab. Stat. 49 (2013), no. 2, 340-373. MR3088373

[8] Amir Dembo and Nike Sun, Central limit theorem for biased random walk on multi-type Galton-Watson trees, Electron. J. Probab. 17 (2012), no. 75, 40. MR2981900 
[9] Andreas Dress, Vincent Moulton, and Werner Terhalle, T-theory: an overview, European J. Combin. 17 (1996), no. 2-3, 161-175, Discrete metric spaces (Bielefeld, 1994). MR1379369

[10] T. Duquesne, The coding compact real trees by real valued functions., Preprint available on $\operatorname{arXiv}(2006)$.

[11] Thomas Duquesne, A limit theorem for the contour process of conditioned Galton-Watson trees, Ann. Probab. 31 (2003), no. 2, 996-1027. MR1964956

[12] Thomas Duquesne, Continuum tree limit for the range of random walks on regular trees, Ann. Probab. 33 (2005), no. 6, 2212-2254. MR2184096

[13] Thomas Duquesne and Jean-François Le Gall, Probabilistic and fractal aspects of Lévy trees, Probab. Theory Related Fields 131 (2005), no. 4, 553-603. MR2147221

[14] Thomas Duquesne and Jean-François Le Gall, Random trees, Lévy processes and spatial branching processes, Astérisque (2002), no. 281, vi+147. MR1954248

[15] Steven N. Evans, Probability and real trees, Lecture Notes in Mathematics, vol. 1920, Springer, Berlin, 2008, Lectures from the 35th Summer School on Probability Theory held in Saint-Flour, July 6-23, 2005. MR2351587

[16] Sébastien Gouëzel and Steven P. Lalley, Random walks on co-compact Fuchsian groups, Ann. Sci. Éc. Norm. Supér. (4) 46 (2013), no. 1, 129-173 (2013). MR3087391

[17] Irene Hueter and Steven P. Lalley, Anisotropic branching random walks on homogeneous trees, Probab. Theory Related Fields 116 (2000), no. 1, 57-88. MR1736590

[18] Svante Janson and Jean-François Marckert, Convergence of discrete snakes, J. Theoret. Probab. 18 (2005), no. 3, 615-647. MR2167644

[19] Steven P. Lalley, The weak/strong survival transition on trees and nonamenable graphs, International Congress of Mathematicians. Vol. III, Eur. Math. Soc., Zürich, 2006, pp. 637647. MR2275700

[20] Steven P. Lalley and Tom Sellke, Hyperbolic branching Brownian motion, Probab. Theory Related Fields 108 (1997), no. 2, 171-192. MR1452555

[21] J.-F. Le Gall, A class of path-valued Markov processes and its applications to superprocesses, Probab. Theory Related Fields 95 (1993), no. 1, 25-46. MR1207305

[22] Jean-François Le Gall, The Brownian cactus II: upcrossings and local times of super-Brownian motion, Probab. Theory Related Fields 162 (2015), no. 1-2, 199-231. MR3350044

[23] Jean-François Le Gall and Shen Lin, The range of tree-indexed random walk in low dimensions, Ann. Probab. 43 (2015), no. 5, 2701-2728. MR3395472

[24] Jean-François Le Gall and Shen Lin, The range of tree-indexed random walk, J. Inst. Math. Jussieu 15 (2016), no. 2, 271-317. MR3480967

[25] Jean-Francois Le Gall and Yves Le Jan, Branching processes in Lévy processes: the exploration process, Ann. Probab. 26 (1998), no. 1, 213-252. MR1617047

[26] Thomas M. Liggett, An improved subadditive ergodic theorem, Ann. Probab. 13 (1985), no. 4, 1279-1285. MR0806224

[27] Thomas M. Liggett, Branching random walks and contact processes on homogeneous trees, Probab. Theory Related Fields 106 (1996), no. 4, 495-519. MR1421990

[28] Russell Lyons and Yuval Peres, Probability on trees and networks, Cambridge Series in Statistical and Probabilistic Mathematics, vol. 42, Cambridge University Press, New York, 2016. MR3616205

[29] Cyril Marzouk, Scaling limits of discrete snakes with stable branching, Ann. Inst. Henri Poincaré Probab. Stat. 56 (2020), no. 1, 502-523. MR4058997

[30] Yuval Peres and Ofer Zeitouni, A central limit theorem for biased random walks on GaltonWatson trees, Probab. Theory Related Fields 140 (2008), no. 3-4, 595-629. MR2365486

[31] Zhan Shi, Branching random walks, Lecture Notes in Mathematics, vol. 2151, Springer, Cham, 2015, Lecture notes from the 42nd Probability Summer School held in Saint Flour, 2012, École d'Été de Probabilités de Saint-Flour. [Saint-Flour Probability Summer School]. MR3444654 
Scaling limits of tree-valued BRW

[32] Andrew Stewart, On the Range of the Random Walk Bridge on the Regular Tree, ProQuest LLC, Ann Arbor, MI, 2016, Thesis (Ph.D.)-University of Toronto (Canada). MR3697600

Acknowledgments. The authors would like to thank the referee and the editor for their helpful suggestions. 